\title{
PD-L1 IncRNA splice promotes lung adenocarcinoma progression via enhancing c-Myc activity
}

Shuang $\mathrm{Qu}^{1,2^{*}}$, Zichen Jiao ${ }^{3^{*}}$, Geng $\mathrm{Lu}^{4^{*}}$, Bing $\mathrm{Yao}^{5}$, Ting Wang ${ }^{6}$, Weiwei Rong ${ }^{1}$, Jiahan $\mathrm{Xu}^{7}$, Ting Fan ${ }^{8}$, Xinlei

Sun $^{1}$, Rong Yang ${ }^{1}$, Jun Wang ${ }^{4}$, Yongzhong Yao ${ }^{7}$, Guifang $\mathrm{Xu}^{8}$, Xin Yan ${ }^{9}$, Tao Wang ${ }^{3+}$, Hongwei Liang ${ }^{1,2+}, \mathrm{Ke}$ $\operatorname{Zen}^{1+}$

From ${ }^{1}$ State Key Laboratory of Pharmaceutical Biotechnology, School of Life Science, Nanjing University, Nanjing, China; ${ }^{2}$ School of Life Science and Technology, China Pharmaceutical University, Nanjing, China; ${ }^{3}$ Department of Cardiothoracic Surgery, Nanjing Drum Tower Hospital, Medical School, Nanjing University, Nanjing, China; ${ }^{4}$ Department of Emergency Medicine, Nanjing Drum Tower Hospital, Medical School, Nanjing University, Nanjing, China; ${ }^{5}$ Department of Medical Genetics, Nanjing Medical University, Nanjing, China ${ }^{6}$ Department of Pathology, ${ }^{7}$ Department of General Surgery, ${ }^{8}$ Department of Gastroenterology, and ${ }^{9}$ Department of Respiratory Medicine, Nanjing Drum Tower Hospital, Medical School, Nanjing University, Nanjing, China

Running title: PD-L1-lnc promotes cancer progression

*These authors contributed equally to this work.

${ }^{+}$Corresponding authors:

Ke Zen, $\mathrm{PhD}$

E-mail: kzen@nju.edu.cn

Hongwei Liang, PhD

E-mail: hwliang@nju.edu.cn

Tao Wang, PhD, MD

E-mail:wangtao_pumc@live.cn 


\begin{abstract}
Although blockade of programmed death-ligand 1 (PD-L1) to enhance T cell immune responses shows great promise in tumor immunotherapy, the efficacy of such immune-checkpoint inhibition strategy is limited for patients with solid tumors. The mechanism underlying the limited efficacy of PD-L1 inhibitors remains unclear. Here, we show that human lung adenocarcinoma, regardless of PD-L1 protein positive or negative, all produce a long non-coding RNA isoform of PD-L1 (PD-L1-lnc) via alternative splicing, which promotes lung adenocarcinoma proliferation and metastasis. PD-L1-lnc in various lung adenocarcinoma cells is significantly upregulated by IFN $\gamma$ in a manner similar to PD-L1 mRNA. Both in vitro and in vivo studies demonstrate that PD-L1-lnc increases proliferation and invasion but decreases apoptosis of lung adenocarcinoma cells. Mechanistically, PD-L1-lnc directly binds to c-Myc and enhances c-Myc transcriptional activity downstream in lung adenocarcinoma cells. Our results provide targeting PD-L1-lnc-c-Myc axis as a novel strategy for lung cancer therapy.
\end{abstract}




\section{INTRODUCTION}

Cancer is one of the major threats to human health worldwide and is responsible for millions of deaths annually [1]. Cancer cells have developed sophistic mechanisms to evade immune surveillance and attack by immune cells. One such mechanism is to express various immune checkpoint molecules, which suppress anti-tumor immunity of immune cells. Among these intrinsic negative checkpoints, programmed death protein 1 (PD-1) and its ligand 1 (PD-L1) are prominent pair and the blockade of PD-1/PD-L1 has led to successful immunotherapies. PD-L1, also known as B7-H1 and CD274, is a transmembrane protein commonly expressed on the surface of tumor cells. PD-L1 specifically binds to its receptor, PD-1, which is expressed on the surface of T cells, B cells and myeloid cells [2]. The binding of PD-L1 to PD-1 is able to activate the downstream signaling of PD-1 receptor in T cells, resulting in inhibition of the proliferation, cytokine generation/release and cytotoxicity of $\mathrm{T}$ cells. The existence of a break on $\mathrm{T}$ cell function may prevent autoimmunity, but many tumor cells exploit this mechanism to protect themselves from immune attack, resulting in tumor immune evasion [3]. The commercial PD-L1 antibodies have shown tremendous success in treating melanoma and blood cancers like leukemia and lymphoma $[4,5]$. However, the efficacy of PD-L1 inhibition is limited for many cancer patients with solid tumors [6-8]. The molecular basis underlying low efficacy of PD-L1 blockade in solid tumor is unclear though several contributing factors have been suggested. First, IFN $\gamma$ fails to induce the expression of PD-L1 protein in certain cancers $[9,10]$. It has been shown that PD-L1 can express in tumor cells as different forms, including membrane PD-L1 (mPD-L1), cellular PD-L1 (cPD-L1) and soluble PD-L1 (sPD-L1)[11, 12]. Given that only mPD-L1 can bind to T cell surface PD-1 and suppress T cell function, low level of mPD-L1 may result in poor efficacy of anti-PD-L1 antibody. These PD-L1 negative tumors had been characterized as 'cold cancer' as opposed to PD-L1 positive tumors, and indeed cold tumors showed low efficacy of PD-L1 blockade treatment [13]. An additional factor contributing limited efficacy of PD-L1 blockade is a lack of infiltration of anti-tumor T cells into tumor microenvironment (TME) due to poor initial antigen presentation $[14,15]$. As PD-L1 executes its function via targeting $\mathrm{T}$ cells, low level of $\mathrm{T}$ cell infiltration would be associated with limited efficacy of PD-L1 blockade.

Long non-coding RNAs (lncRNAs) have recently gained attention in the field of cancer research [16-18]. As 
RNA transcripts of 200 or more nucleotides that are not translated to proteins, lncRNAs can be transcribed from their own promotors, from the promotors of other coding or non-coding sequences of DNA or from the enhancer sequences. Some lncRNAs are derived through alternative splicing of transcribed RNA, a process which occurs in over $90 \%$ of human multi-exon protein-coding genes [19]. Several classes of lncRNAs have been discovered on the basis of diverse parameters such as transcript length, association with annotated protein-coding genes and mRNA resemblance among others [20]. Recent discoveries suggest that lncRNAs are relevant to cancer progression whereby lncRNAs interact with both oncogenic and tumor suppressive pathways [16]. In line with this, the expression of lncRNAs has been widely reported to be dysregulated in various human cancers $[17,18]$. Despite these findings, the biogenesis and regulatory role of lncRNAs in cancer development, however, remains incompletely understood.

In the present study, we report that, in addition to PD-L1 mRNA, human lung adenocarcinoma PD-L1 gene can generate a long non-coding RNA (PD-L1-lnc) through alternative splicing. Moreover, in a similar manner to PD-L1 mRNA, PD-L1-lnc in lung adenocarcinoma is markedly upregulated by IFN $\gamma$. Once generated, PDL1-lnc promotes lung adenocarcinoma cell proliferation and invasion but decreases cell apoptosis via directly binding to c-Myc and activating c-Myc transcriptional activity. Taken together, this study identifies PD-L1lnc-c-Myc axis as a novel mechanism underlying human lung adenocarcinoma progression. 


\section{MATERIALS AND METHODS}

\section{Clinical samples}

The 275 pairs of lung adenocarcinoma tissues and adjacent non-cancerous tissues were collected from patients who were diagnosed with lung adenocarcinoma at the Nanjing Drum Tower Hospital (Nanjing, China) and who had not yet received treatment. The clinic pathological features are described in Table S1. Tissues were collected after surgical resection and stored in liquid nitrogen before use. The study was authorized by the Ethics Committee of the Nanjing Drum Tower Hospital. All experiments were performed in accordance with relevant guidelines and regulations.

\section{Cell lines, culture conditions and IFN $\gamma$ treatment}

Human lung cancer cell lines A549, PC9, H1975, H1299 and H1650 were purchased from American Type Culture Collection (ATCC) (Manassas, VA, USA). Cells were cultured in Dulbecco's Modified Eagle's Medium (DMEM, Gibco, Carlsbad, CA, USA) supplemented with 10\% (v/v) FBS (Gibco, Carlsbad, CA, USA), $1 \%(\mathrm{v} / \mathrm{v})$ penicillin/streptomycin (Gibco, Carlsbad, CA, USA). For IFN $\gamma$ treatment, cells were seeded into six-well plates on day 1 , targeting $70 \%-80 \%$ of confluence on the day of surface staining. On day 2 , cells were exposed to $100 \mathrm{ng} / \mathrm{mL}$ IFN $\gamma$ (PeproTech, USA) for $24 \mathrm{~h}$.

\section{Immunohistochemical staining}

Tissues were fixed in $4 \%$ paraformaldehyde, embedded in paraffin, and cut into $4 \mu \mathrm{m}$ sections. Immunohistochemical staining of PD-L1 was performed using the anti-PD-L1 antibody (SP142, Zhongshanjinqiao, China) according to the manufacturer's protocol.

\section{RNA extraction and RT-PCR/qRT-PCR assay}

Total RNAs were isolated by TRIzol ${ }^{\mathrm{TM}}$ Reagent (Invitrogen, US) according to the manufacturer's instruction. The quality and quantity of RNA was assayed by Nanodrop 2000 spectrophotometer (Thermo Fisher Scientific, USA). The nuclear and cytoplasmic fractions were purified by PARIS Kit (Ambion, Life Technologies, USA). RNA was reverse transcribed by HiScript II Q RT SuperMixfor qPCR (+gDNA wiper) (Vazyme, Nanjing, 
China). The $1.1 \times$ T3 Super PCR Mix (TsingKe, Nanjing, China) was used for PCR. The 10\% vertical polyacrylamide electrophoresis was performed to observe the cDNA PCR products. AceQ qPCR SYBR Green Master Mix (Vazyme, Nanjing, China) was used for qRT-PCR, and GAPDH was used to normalize the level of PD-L1-lnc and PD-L1 mRNA. Primers were listed in table S2. The purified fragments were cloned into the pCR $®$ II TA vector using the TA cloning kit (Thermo Fisher Scientific, United States) and sequenced at the TsingKe Biotechnology (Nanjing, China) to validate the cDNA PCR products.

\section{RNA BaseScope}

BaseScope $^{\mathrm{TM}}$ Probe for PD-L1-lnc and PD-L1 mRNA were designed and synthesized by ACD (Cat. No.700001, 700001-C2, Advanced Cell Diagnostics, CA, USA). Tissues and cells were fixed by 10\% neutral buffered formalin on slides for detection of PD-L1-lnc and PD-L1 mRNA. The signals of the PD-L1-lnc and PD-L1 mRNA probes were detected by BaseScope ${ }^{\mathrm{TM}}$ Detection Reagent Kit (ACD, USA) according to the manufacturer's instructions. The images were acquired on Leica SP5 Scanning Laser Confocal Microscope (Leica Microsystems, Wetzlar, Germany).

\section{Flow cytometry}

Flow cytometry was performed using a CytoFLEX S (Beckman Coulter Life Sciences, Mississauga, ON).

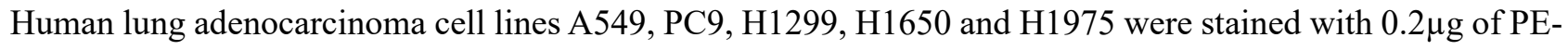
conjugated anti-PD-L1 (Biolegend, USA) antibody.

\section{Western blotting}

The protein extraction reagent (Thermo Scientific) with a cocktail of proteinase inhibitors (Roche Applied Science, Switzerland) was used to isolate the total protein from cells or tissue samples. Equal amount of total protein was separated by $10 \%$ SDS-PAGE and transferred onto a PVDF membrane. Then, the membranes were blocked with $5 \%$ skimmed milk and incubated the membranes with primary antibodies at $4{ }^{\circ} \mathrm{C}$ overnight and then incubated with secondary antibodies at room temperature for $2 \mathrm{~h}$. The bands were examined by Immobilob ${ }^{\mathrm{TM}}$ Western Chemiluminescent HRP Substrate (Millipore, Billerica, MA, USA). The primary 
antibody and secondary antibodies detailed information list below: PD-L1 [E1L3N®] XP ${ }^{\circledR}$ Rabbit mAb (\#13684, Cell Signaling Technology, Beverly, MA, USA), GAPDH (6C5) Mouse mAb (sc-32233, Santa Cruz, USA), Histone-H3 Rabbit Polyclonal antibody (17168-1-AP, Proteintech, Wuhan, China), c-MYC Rabbit Polyclonal antibody (10828-1-AP, Proteintech, Wuhan, China), MAX antibody [EPR19352] (ab199489, abcam, US ), Rabbit (DA1E) mAb IgG XP® Isotype Control (\#3900, Cell Signaling Technology, Beverly, MA, USA) and the secondary antibodies (goat anti-rabbit IgG-HRP, sc-2030, Santa Cruz; goat anti-mouse IgG-HRP, sc-2005, Santa Cruz; Mouse Anti-rabbit IgG-HRP [L27A9] mAb „\#5127, Cell Signaling Technology).

\section{Cell proliferation assay}

To measure the proliferation rate of human lung cancer cells, EdU assays were performed. Briefly, A549 and PC9 cells were seeded in 6-well plates and transfected with vectors. At $24 \mathrm{~h}$ after transfection, cells were harvested and reseeded 48-well plates for EdU assays. The EdU assay kit (RiBoBio, China) was used to determine the proliferation rate of cells according to the manufacturer's instructions.

\section{Cell invasion assay}

Firstly, the upper chamber of transwell (Millipore, USA) was coated with diluted Matrigel $(200 \mathrm{mg} / \mathrm{mL}, \mathrm{BD}$ Biosciences, MA) at the density of $50 \mu \mathrm{L} /$ well before use. A549 and PC9 cells were transfected with vectors. After $24 \mathrm{~h}$, cells were resuspended in FBS-free DMEM medium and reseeded on the upper surface of 24-well plates. Cells were allowed to migrate across the $8-\mu \mathrm{m}$ membrane toward medium with $20 \%$ FBS for $24 \mathrm{~h}$. Then, the cells were fixed with $4 \%$ paraformaldehyde and dyed with $0.5 \%$ crystal violet. Non-migrating cells were removed using a cotton swab. The migrant cells were blindly counted under a light microscope (Leica Microsystems, Wetzlar, Germany).

\section{Cell apoptosis assay}

The apoptosis of A549 and PC9 cells was assayed using the Annexin V-Alexa Fluor 647/PI (YEASEN, China) based on the procedures provided by the manufacturer. The transfected A549 and PC9 cells were cultured in serum-free DMEM for $24 \mathrm{~h}$. The collected cells were washed with cold PBS and re-suspended in $1 \times$ binding 
buffer, followed by staining with Alexa Fluor 647-Annexin V and propidium iodide (PI) in the dark for 15 min. The apoptotic cells were calculated using flow cytometer (Beckman Coulter Life Sciences, Mississauga, ON).

\section{Xenograft assays in nude mice}

All animal care and handling procedures were performed in accordance with the National Institutes of Health's Guide for the Care and Use of Laboratory Animals. Male athymic BALB/c nude mice (6 weeks old) were purchased from the Model Animal Research Center of Nanjing University (Nanjing, China) and were randomly divided into 3 groups (5 mice per group). A549 cells were firstly transfected with control vector, vector expressing PD-L1-lnc (PD-L1-lnc) or PD-L1-lnc shRNA (PD-L1-lnc shRNA), respectively. Cells were then selected using $500 \mu \mathrm{g} / \mathrm{mL} \mathrm{G} 418$ (ThermoFisher) or $1 \mu \mathrm{g} / \mathrm{mL}$ Puromycin (ThermoFisher) for 2 weeks. Three stable lung cancer cell lines were then subcutaneously injected into mice $\left(10^{6}\right.$ cells $/ 0.1 \mathrm{ml}$ PBS per mouse). The needle was inserted into the armpit of the left foreleg at a 45 degree angle and a $5 \mathrm{~mm}$ depth, midway down. Then, the longest diameter (a) and the shortest diameter (b) of the tumor were measured using digital calipers every three days, and the tumor volume $(V)$ was calculated according to formula: $V=a \times b^{2} / 2$. Mice were sacrificed and photographed 15 days post-injection. The xenograft tumors were removed and analyzed. Tissues were subjected to extraction of total RNA, as well as hematoxylin and eosin (H\&E) staining and Ki67 immunohistochemical staining.

\section{Vector construction and cell transfection}

To overexpress PD-L1-lnc, the full-length cDNA of PD-L1-lnc was synthesized and cloned into pcDNA3.1P2A-eGFP vector (GenScript, China). To suppress PD-L1-lnc, the PD-L1-lnc shRNA vectors were synthesized and then cloned into pLKO.1 vector (GenScript, China). The siRNA target sequences were listed in table S3. Cells were transfected using Lipofectamine 3000 (Invitrogen, USA) according to the manufacturer's instruction.

\section{Microarray analysis}

Total RNAs were isolated from the A549 cells transfected with PD-L1-lnc overexpressed vector or control 
vector by TRIzol reagent and purified by RNeasy Mini Kit (Qiagen, USA). RNA samples were performed to Microarray analysis by Agilent SurePrint G3 human gene expression Microarray 8X60K (Agilent Technologies). After hybridization, slides were scanned on the Agilent Microarray Scanner. Data were extracted using Feature Extraction software 10.7 (Agilent Technologies). Raw data were normalized by Quantile algorithm, limma package the R program. Significant differential expressed transcripts were defined as fold change $\geq 2$ or $\leq-2$ and P-value $\leq 0.05$.

\section{Streptavidin pull down of PD-L1-Inc and c-Myc protein}

For each pull down sample $100 \mu 1$ of streptavidin magnetic beads (S1420S, New England Biolabs, US) were washed with wash/binding buffer $(0.5 \mathrm{M} \mathrm{NaCl}, 20 \mathrm{mM}$ Tris-HCl, $\mathrm{pH} 7.5,1 \mathrm{mM}$ EDTA) twice, then incubation with probes for 1 hour at $4^{\circ} \mathrm{C}$. For biotin-coupled RNA capture, the 5'-end biotinylated PD-L1-lnc probe or control RNA were used. Probes used as follows: PD-L1-lnc probe: 5'-3' CATCCATCATTCTCCCA AGTGAGTCCT, GFP probe: 5'-3' TGAAGTTCACCTTGATGCCGTTCTTCT GCTTGTCGGCCATGATAT AGACGTTGTGGCTGT. The $0.5 \mathrm{ml}$ cell lysis buffer (Invitrogen, USA) with complete protease inhibitor cocktail (Roche Applied Science, IN) and Recombinant RNase Inhibitor (Takara, Japan) were added into the cell pellets, and lysed by sonication. The cell lysates incubation with RNA-coupled beads followed by centrifugation at $13,000 \times \mathrm{rpm}$ for $20 \mathrm{~min}$. After rotating for $4 \mathrm{~h}$ at $4^{\circ} \mathrm{C}$, the beads were washed with $1 \mathrm{ml}$ lysis buffer (containing $300 \mathrm{mM} \mathrm{NaCl}$ ) twice, $1 \mathrm{ml}$ low-salt lysis buffer (containing $150 \mathrm{mM} \mathrm{NaCl}$ ). Half of beads were resuspended in TRIzol ${ }^{\mathrm{TM}}$ Reagent for detecting PD-L1-lnc level, while half of beads were resuspended in RIPA lysis buffer for detecting c-Myc protein level.

\section{RNA immunoprecipitation (RIP)}

A549 Cells transfected with PD-L1-lnc overexpressed vector or control vector were lysed with lysis buffer (20 mM Tris- $\mathrm{HCl}, 150 \mathrm{mM} \mathrm{NaCl}, 0.5 \%$ Nonidet P-40, 2 mM EDTA, $0.5 \mathrm{mM}$ DTT, $1 \mathrm{mM} \mathrm{NaF}, 1 \mathrm{mM}$ PMSF and $1 \%$ Protease Inhibitor Cocktail from Sigma, $\mathrm{pH}$ 7.4) for $30 \mathrm{~min}$ on ice. After cleared by centrifugation $(16,000 \mathrm{~g})$ for $10 \mathrm{~min}$ at $4{ }^{\circ} \mathrm{C}$, the lysates were subjected to immunoprecipitation with anti-cMyc antibody or IgG followed by protein A/G-Agarose beads. After the elution, the proteins were isolated by RIPA lysis buffer 
for western blot assays and the RNA were isolated with TRIzol ${ }^{\mathrm{TM}}$ Reagent for detecting PD-L1-lnc level.

\section{In vitro mRNA stability assay}

To monitor the effect of PD-L1-lnc on the stability of PD-L1 mRNA, A549 cells transfected with control vector and PD-L1-lnc vector were treated by $5 \mu \mathrm{g} / \mathrm{ml}$ actinomycin D to block the transcription of new PD-L1 mRNA, and then assessed PD-L1 mRNA level at $0,3,6,9,12$ or $24 \mathrm{~h}$ post-treatment by qRT-PCR.

\section{Statistical analysis}

All experiments were performed in triplicate or as indicated in the experiments. Data were presented as the mean \pm SD. When only two groups were compared, Student's $t$-test was used. Comparisons involving multiple dependent measures were Tukey-Kramer corrected. The reported $P$ value was 2 -sided. The differences with ** $\mathrm{P}<0.05$ were considered significantly different. 


\section{RESULTS}

\section{Generation of PD-L1 IncRNA via alternative splicing in PD-L1 protein positive or negative human lung} adenocarcinoma tissues, as well as various lung adenocarcinoma cells

By immunohistochemical staining, we screened more than 275 human lung adenocarcinoma samples and their paired distal non-cancerous tissues samples from patients registered in Nanjing Drum Tower Hospital, Nanjing University School of Medicine (Nanjing, Jiangsu, China) from 2017-2018 (Table S1) for PD-L1 protein expression. As shown in fig. S1A and Table S1, the majority of lung cancer samples were PD-L1 negative, with little or no PD-L1 protein expression, which is in line with the previous report about the PD-L1 protein expression in Chinese lung cancer patients [21]. Although the percentage of PD-L1-positive lung cancers at advanced stage was higher than that of lung cancers at early stage [22], considerable numbers of lung adenocarcinoma samples at advanced stages expressed little or no PD-L1 protein (fig. S1A and Table S1).

To explore the mechanism underlying the variety of PD-L1 protein expression in lung adenocarcinoma at various stages, we detected the PD-L1 mRNA level in PD-L1 protein-positive or PD-L1 protein-negative lung cancer tissues. To our surprise, analysis of PCR end-product identified two bands at 198bp and 92bp, respectively (Fig. 1A, left). Sanger sequencing showed that 198bp band matched to the sequence of PD-L1 mRNA (Fig. 1A, right upper), while 92bp band was a noncoding isoform (NR_052005.1) which lacks an alternate internal segment (named as PD-L1-lnc) (Fig. 1A, right lower). To confirm these results, another pair of probes for detecting the two-missing parts was designed (Fig. 1B, upper). The results of agarose gel showed two bands at $878 \mathrm{bp}$ and $705 \mathrm{bp}$, respectively (Fig. 1B, lower left). The $878 \mathrm{bp}$ band matched the sequence of PD-L1 mRNA (Fig. 1B, upper right; fig. S2A), while the 705bp band matched to the NR_052005.1 missing 106nt in exon 4 and 67nt between exon 5 and 6 (Fig. 1B, lower right; fig. S2B). Furthermore, RNA BaseScope analysis [23] was performed to examine the co-expression of PD-L1 mRNA and PD-L1-lnc in human lung cancer tissues. As shown in Fig. 1C, a clear co-existence of PD-L1 mRNA (blue dots) and PD-L1-lnc (red dots) in lung cancer tissues was observed. To accurately quantify these RNA fragments, the specific probes for PD-L1 mRNA and PD-L1-lnc were designed (Fig. 1D, upper). The PCR products of these probes (for PDL1 mRNA and PD-L1-lnc) were confirmed by agarose gel (Fig. 1D, lower left) and Sanger sequencing (fig. 
S3A-B), respectively. The qRT-PCR assay with specific probes detected similar amount of PD-L1 mRNA (Fig. 1D, lower middle) and PD-L1-lnc (Fig. 1D, lower right) in PD-L1 protein-positive and PD-L1 protein-negative tumor samples.

Expression of PD-L1-lnc and PD-L1 mRNA was next detected in various human lung cancer cell lines, including A549, PC9, H1975, H1650 and H1299. Western blotting and flow cytometry analysis confirmed various levels of PD-L1 on the lung adenocarcinoma cell lines (Fig. 2A-B). Similar to that in human lung adenocarcinoma tissues, both PD-L1-lnc and PD-L1 mRNA were detected in various lung cancer cells in various human lung cancer cells by agarose gel through PD-L1 mRNA/lnc primer 2 (Fig. 2C), RNA BaseScope (Fig. 2D) and RT-PCR by PD-L1 mRNA specific primer 3 or PD-L1-lnc specific primer 4 (Fig. 2E).

To further investigate whether the PD-L1-lnc exist in other cancers in addition to LUAD, we analyzed the RNA-seq data in TCGA database. The results showed the expression of PD-L1-lnc in various cancers, including BRAC, ESCA and STAD, etc. (fig. S4A). Pan-cancer analysis in TCGA database further indicated a negative association of PD-L1-lnc level with cancer patient survival rates (fig. S4B). To confirm these results derived from TCGA database, we used specific probes to detect both PD-L1 mRNA and PD-L1-lnc in multiple cancer cell lines and cancer tissues (fig. S4C, left). The PCR products confirmed the expression of PD-L1-lnc in various tumor cells and tissues (fig. S4C, right).

We next linked PD-L1-lnc to GFP mRNA in an expressing system to confirm PD-L1-lnc as a non-coding RNA (fig. S5A, upper). RT-PCR assay and fluorescence microscopy showed a high level of GFP mRNA (fig. S5A, lower) but no GFP protein in the PD-L1-lnc-expressing system (fig. S5B), confirming PD-L1-lnc as a lncRNA.

\section{Lung cancer cell PD-L1-Inc is markedly upregulated by IFN $\gamma$ in a similar manner to PD-L1 mRNA}

The expression of PD-L1 mRNA and protein in tumor cells can be upregulated by T cell-secreted IFN $\gamma$ [24], which provides a mechanism for cancer to suppress T cell immune responses via ligating T cell surface PD-1. Given that PD-L1-lnc is spliced from 638 to 744 and 832 to 899 nucleotides downstream of the splicing site 
of PD-L1 mRNA transcription (Fig. 3A), we anticipated that IFN $\gamma$ would enhance PD-L1-lnc transcription, in a similar manner to that of PD-L1 mRNA. To test this, we treated A549, PC9, H1975, H1650 and H1299 cells with or without IFN $\gamma$ followed by detection of PD-L1 protein, PD-L1 mRNA and PD-L1-lnc levels. Western blot analysis showed that IFN $\gamma$ treatment strongly enhanced PD-L1 protein level in the cells (fig. S6A). Increase of PD-L1 protein level on cancer cell surface was also shown by flow cytometry analysis (fig. S6B). In agreement with previous report [25], IFN $\gamma$ treatment strongly increased PD-L1 mRNA level in A549, PC9, H1975, H1650 and H1299 cells (Fig. 3B). In a similar manner, IFN $\gamma$ treatment also significantly increased PD-L1-lnc level in all the lung cancer cell lines (Fig. 3B). To further examine the distribution of PD-L1-lnc in lung cancer cells, we purified nuclear and cytoplasm fractions from A549 cells. As shown in Fig. 3C, left, isolated nuclear and cytoplasm fractions exhibited high levels of marker proteins, Histone H3 and GAPDH, respectively, confirming the enrichment of nuclear and cytoplasm fraction in isolated products. Agarose gel (Fig. 3C, left) and RT-PCR analysis (Fig. 3C, right) demonstrated that PD-L1-lnc and PD-L1 mRNA in both nuclear and cytoplasm fractions were upregulated by IFN $\gamma$ treatment.

\section{PD-L1-Inc promotes proliferation and invasion but suppresses apoptosis of lung cancer cells}

It has been reported that lncRNAs derived from gene alternative splicing can protect the corresponding gene mRNA against the nonsense-mediated decay (NMD) pathway, which targets mRNAs harboring premature termination codons for degradation [26]. Since PD-L1-lnc use the same 5'-most supported translational start codon as the PD-L1 mRNA, the PD-L1-lnc was rendered a candidate for nonsense-mediated mRNA decay to protect PD-L1 mRNA. To test this, we firstly compared the expression level of PD-L1-lnc and PD-L1 mRNA in our collected lung cancer tissues and lung adenocarcinoma data set from TCGA. The results showed there was no correction between PD-L1-lnc and PD-L1 mRNA in both our collected lung cancer tissues and lung adenocarcinoma data set from TCGA (fig. S7A-B). Moreover, we also analyzed associations between PD-L1lnc or PD-L1 mRNA and overall survival of lung adenocarcinoma based on the TCGA database. Notably, the Kaplan-Meier survival analysis showed that lung adenocarcinoma patients with high PD-L1-lnc expression had shorter overall survival (fig. S7C), while the expression level of PD-L1 mRNA had no effect on the overall survival of lung adenocarcinoma patients (fig. S7D). In order to further explore the relationship between PD- 
L1-lnc or PD-L1 mRNA, we overexpressed PD-L1-lnc by PD-L1-lnc vector or depleted PD-L1-lnc by PDL1-lnc shRNA in lung cancer cells and then monitored the cellular levels of PD-L1 mRNA and protein. As showed in fig. S7E, the expression level of PD-L1 mRNA had no change despite PD-L1-lnc was significantly upregulated or downregulated. Western blotting and Flow cytometry analysis also showed overexpression or depletion of PD-L1-lnc in A549 cells had no effect on the protein level of PD-L1 (fig. S7F-G). We further monitored the effect of PD-L1-lnc on the stability of PD-L1 mRNAs, the decay of PD-L1 mRNAs in A549 cells transfected with control vector and PD-L1-lnc vector was assessed by qRT-PCR after blocking the transcription of new PD-L1 mRNAs via actinomycin D (ActD) treatment. As shown in fig. S6H, the relative level of PD-L1 mRNAs in A549 cells transfected with control vector and PD-L1-lnc vector displayed no difference. Furthermore, the effect of PD-L1-lnc on the expression of PD-L1 mRNA and protein during IFN $\gamma$ stimulation was also investigated. Both flow cytometry and Western blots analysis showed that PD-L1-lnc had no effect on the PD-L1 protein level in A549 cells before or after IFN $\gamma$ treatment (fig. S7I-J). Taken together, inconsistent with our assumptions and the prediction by NCBI database, PD-L1-lnc exhibited no effect on PDL1 mRNA expression.

Although PD-L1-lnc could not enhance the expression of PD-L1 mRNA and protein, we found that it strongly affected lung cancer progression. In these experiments, cancer cell proliferation, invasion and apoptosis were assessed using EdU staining [27], Transwell assay [28] and Annexin V labeling [29], respectively. As shown in Fig. 4, overexpression of PD-L1-lnc in A549 and PC9 lung cancer cells strongly enhanced cell proliferation (Fig. 4A) and invasion (Fig. 4B) but suppressed tumor cell apoptosis (Fig. 4C) compared to untreated cancer cells. In contrast, when PD-L1-lnc in lung cancer cells was depleted via transfection with specific PD-L1-lnc shRNA, the proliferation (Fig. 4A) and invasion (Fig. 4B) of cancer cells were significantly reduced, whereas the apoptosis of cancer cells (Fig. 4C) was increased, compared to untreated cancer cells.

Effects of PD-L1-lnc on the progression of lung cancer cells were further validated in lung cancer xenograft mouse model. For this experiment, we developed two lung cancer cell lines: A549 cells which stably express high level of PD-L1-lnc (PD-L1-lnc) and A549 cells with PD-L1-lnc depleted (PD-L1-lnc shRNA). These two 
lines of cancer cells, as well as A549 cells transfected with control vector as the negative control, were subcutaneously injected into athymic nude mice. Although no significant difference in body weight was observed in three groups of mice during the time frame of experiment (Fig. 5A), tumor size measurement showed a markedly different growth rate among these lung cancer cells (Fig. 5B-C). Specifically, A549 cells expressing higher level of PD-L1-lnc grew significantly faster than control A549 cells, whereas A549 cells with PD-L1-lnc depletion showed a growth delay compared to control A549 cells. In line with this, Ki67 staining analysis in tumor tissue sections showed that A549 cells expressing higher level of PD-L1-lnc had a higher proliferation rate whereas A549 cells with PD-L1-lnc depletion had a lower proliferation rate than control A549 cells (Fig. 5D). Analysis of PD-L1-lnc level in the implanted tumor tissues by RT-PCR confirmed that, compared to control A549 lung cancer tissue, PD-L1-lnc A549 tumor and PD-L1-lnc shRNA A549 tumor expressed significantly higher and lower level of PD-L1-lnc, respectively (Fig. 5E). In contrast, PD-L1 mRNA levels in both PD-L1-lnc and PD-L1-lnc shRNA A549 tumors were not different.

\section{PD-L1-Inc executes its oncogenic function through activating c-Myc signaling}

To explore the mechanism underlying the pro-tumor function of PD-L1-lnc, we performed microArray analysis of human genes in A549 cells which stably transfected with PD-L1-lnc-expressing vector (PD-L1-lnc) and A549 cells transfected with control vector. As shown in Fig. 6A, we found that 2640 genes were upregulated while 1810 genes were downregulated (fold-change >2) in A549 cells after PD-L1-lnc overexpression. Further analysis of upregulated or downregulated genes compared to control A549 cells indicated that majority genes ( $20.9 \%$ ) belonged to the c-Myc pathway regulation (Fig. 6B). Next, using qRT-PCR, we examined a panel of these genes that both displayed significant alteration in microArray analysis and were in the c-Myc-regulating network. As shown in Fig. 6C, PD-L1-lnc overexpression or depletion markedly altered these genes in both A549 cells and lung cancer xenograft (Fig. 6C). These results indicated PD-L1-lnc might promote tumor progress through c-Myc.

LncRNAs have been confirmed to play important roles in regulating tumorigenesis through interaction with proteins. We used two strategies to examine the potential interactions between PD-L1-lnc and c-Myc, a main 
member of MYC family that plays a critical role in cancer cell proliferation, metastasis and apoptosis resistance [30]. As shown in Fig. 6D, left, we designed a system consisting of biotin-conjugated antisense oligonucleotide of PD-L1-lnc and streptavidin-conjugated magnetic beads to specifically pull-down PD-L1-lnc. Biotinconjugated scramble oligonucleotide was used as a negative control. Western blot analysis showed that c-Myc was immunoprecipitated in association with PD-L1-lnc, whereas no c-Myc was detected in immunoprecipitated complex by scramble oligonucleotide (Fig. 6D, right). We also immunoprecipitated cMyc in A549 cells that were overexpressed with PD-L1-lnc or control A549 cells using anti-c-Myc antibody followed by Protein A/G-conjugated Sepharose beads (Fig. 6E, left). As shown in Fig. 6E, right, PD-L1-lnc was detected in the immunoprecipitated product from the control A549 cells and PD-L1-lnc overexpressed A549 cells by anti-c-Myc antibody, whereas no PD-L1-lnc was detected in immunoprecipitated complex by IgG. Moreover, there was much more PD-L1-lnc in the immunoprecipitated product from PD-L1-lnc overexpressed A549 cells, compared to the control A549 cells. Above all, our results suggested the association of PD-L1-lnc with c-Myc in A549 cells.

The interactions between PD-L1-lnc and c-Myc were next analyzed using catRAPID omics [31] and RPISeq [32] algorithms. The interaction probabilities of PD-L1-lnc and c-Myc predicted by both algorithms were more than 0.90, and the most probable binding area of c-Myc in PD-L1-lnc was located in 500-1000nt of PD-L1lnc. To explore the PD-L1-lnc tertiary conformation, we first determined its secondary structures using minimum free energy algorithm implemented in Mfold (version 2.3) [33]. The results showed that PD-L1-lnc might form hairpin structure, likely from 500nt to 1000nt. This secondary structure with a lower theoretical value of free energy was then selected as a model for 3D structure prediction. The output file containing primary sequence and an associated secondary structure (Dot-Bracket Notation) was submitted to RNA Composer to generate the 3D structure [34]. NPDock [35] was then used to construct the in-silico molecular docking between PD-L1-lnc and c-Myc. The c-Myc 3D structure used in the docking procedure was derived from Protein Data Bank. As showed in Fig. 7A, c-Myc could bind to the double helix structure of hairpin structure formed by PD-L1-lnc. Further analysis showed that hydrogen bonds might be formed between the $\mathrm{ASN}^{100}$ of c-Myc and the AG $\mathrm{G}^{901-902}$ of PD-L1-lnc, the GLY ${ }^{103}$ of c-Myc and the $\mathrm{U}^{883}$ of PD-L1-lnc, the PHE ${ }^{107}$ 
of c-Myc and the $\mathrm{G}^{882}$ of PD-L1-lnc, the $\mathrm{ALA}^{110}$ of c-Myc and the $\mathrm{U}^{881}$ of PD-L1-lnc, the THR ${ }^{117}$ of c-Myc and the $\mathrm{AU}^{880-881}$ of PD-L1-lnc, the GLU ${ }^{118}$ of c-Myc and the $\mathrm{G}^{902}$ of PD-L1-lnc, the GLY ${ }^{121}$ of c-Myc and the $\mathrm{G}^{877}$ of PD-L1-lnc, respectively (fig. S8). These hydrogen bonds could enable the formation of a basic helixloop-helix Zip motif [36] in c-Myc. To validate these predicted binding sites, two PD-L1-lnc vectors, in which the predicted binding sites were mutated (PD-L1-lnc mut) or depleted (PD-L1-lnc del), were constructed (Fig. 7B). These mutant PD-L1-lnc vectors as well as the WT PD-L1-lnc vector were then transfected into A549 cells. As shown in fig. S9A-B, both WT PD-L1-lnc, PD-L1-lnc del and PD-L1-lnc mut vectors expressed PDL1-lnc along with its linked GFP mRNA without affecting PD-L1 mRNA and protein. Immunoprecipitation assay using biotin-conjugated antisense oligonucleotide of GFP mRNA plus streptavidin-conjugated magnetic beads showed that c-Myc was associated with wild type PD-L1-lnc but not mutant PD-L1-lnc (Fig. 7C). In line with this, direct pulldown of c-Myc using anti-c-Myc antibody plus Protein A/G-conjugated Sepharose beads in A549 cells transfected with WT PD-L1-lnc-GFP mRNA or mutant PD-L1-lnc-GFP mRNA indicated that only WT PD-L1-lnc-GFP mRNA but not mutant PD-L1-lnc-GFP mRNA was associated with c-Myc (Fig. 7D).

Previous studies showed that helix-loop-helix Zip motif of c-Myc enables formation of a heterodimer with chaperone protein Max in initiating gene transcription. Given that binding of PD-L1-lnc with c-Myc can facilitate c-Myc to form a basic helix-loop-helix Zip motif (Fig. 7A), we postulate that PD-L1-lnc can enhance c-Myc transcriptional activity through enabling a c-Myc conformation change which allows for a heterodimer formation with Max. To test the hypothesis, we compared the protein level of Max in the immunoprecipitated product by anti-c-Myc antibody in the A549 cells transfected the PD-L1-lnc-expressing vector or control vector. As expected, the protein level of Max significantly increased in the A549 cells transfected with the PD-L1lnc-expressing vector, compared to the A549 cells transfected with the control vector (Fig. 7E). Immunoprecipitation and Western blot analysis further indicated that overexpression of PD-L1-lnc in A549 cells only enhanced the nuclear distribution of Max which were associated with c-Myc, not the total cellular level of c-Myc-associated Max. Supporting the notion that PD-L1-lnc binds to c-Myc, immunofluorescence labeling showed that overexpression of PD-L1-lnc significantly enhanced nuclear translocation of c-Myc in 
A549 cells (Fig. 7F). Cell fraction and Western blot analysis indicated the increase of nuclear (Nucl) distribution of c-Myc by overexpression of PD-L1-lnc whereas the total cell level of c-Myc remained unchanged (Fig. 7G). These results suggest that PD-L1-lnc may enhance c-Myc activity by enhancing the binding with MAX and promoting the entry of c-Myc into nuclei.

To further test whether PD-L1-lnc executes its function through c-Myc signaling pathway, we knocked down c-Myc expression via c-Myc siRNA in A549 cells (fig. S10) in which PD-L1-lnc was or was not overexpressed and then monitored the cell proliferation, invasion and apoptosis. As shown in Fig. 8A-C, overexpression of PD-L1-lnc strongly promoted A549 cell proliferation (Fig. 8A), invasion (Fig. 8B) and resistance to apoptosis (Fig. 8C). Depleting cellular c-Myc via c-Myc siRNA treatment, however, largely abolished the effect of PDL1-lnc on enhancing A549 cell proliferation (Fig. 8A), invasion (Fig. 8B) and resistance to apoptosis (Fig. 8C). The function of PD-L1-lnc mutants which do not bind to c-Myc was also investigated. As shown in Fig. 8AC, transfection with PD-L1-lnc mutants did not enhance cell proliferation, invasion and resistance to apoptosis. 


\section{DISCUSSION}

As an important immune checkpoint, PD-L1 suppresses T cell immune responses via binding to T cell surface PD-1 and initiating T cell program death [37, 38]. Tumor cells often upregulate PD-L1 expression especially under the condition of $\mathrm{T}$ cell infiltration, which provides a mechanism for tumor cells to evade the attack by $\mathrm{T}$ cells. Blockade of PD-L1/PD-1 interactions has thus been widely applied in tumor immunotherapy and achieved great success in treating various tumors particularly melanoma, leukemia and lymphoma. However, the efficacy of PD-L1 blockade strategies is limited for solid cancers [6-8]. In the present study, we demonstrate that PD-L1 gene in lung adenocarcinoma tissues, as well as various lung cancer cells, produces another PDL1 transcript, PD-L1-lnc, via PD-L1 gene alternative splicing. In a similar manner to PD-L1 mRNA, PD-L1lnc level is markedly increased by IFN $\gamma$. Moreover, PD-L1-lnc strongly promotes tumor cell proliferation, survival and invasion via enhancing c-Myc activity. Given that production of PD-L1-lnc in lung cancer cells is not dependent upon PD-L1 protein expression and depletion of PD-L1-lnc markedly suppresses tumor growth both in vitro and in vivo, depleting tumor cell PD-L1-lnc may provide a novel anti-tumor therapeutic approach in addition to PD-L1 protein blockade.

The efficacy of immune checkpoint blockade in tumor therapy is dependent on the infiltration and activation of anti-tumor T cells in tumor microenvironment, which leads to significant elevation of IFN $\gamma$ level [39]. Although IFN $\gamma$ had been regarded as an anti-tumor cytokine [40, 41], recent studies showed a 'double-edge' effect of IFN $\gamma$ in tumor progression $[42,43]$. For example, IFN $\gamma$ can upregulate immune checkpoint molecules particularly PD-L1, which enhances tumor immune evasion $[24,44]$. Here we showed that, in addition to PDL1 mRNA, IFN $\gamma$ also enhanced expression of PD-L1-lnc. Judged by the PD-L1 gene structure, it seems that PD-L1-lnc and PD-L1 mRNA are upregulated by IFN $\gamma$ through a similar mechanism.

Previous studies have reported the expression of PD-L1 is modulated by alternative splicing [11, 45]. Zhou et al. identified four PD-L1 splicing variants that lack the transmembrane domain, and these secretory forms of PD-L1 can also suppress the activities of both $\mathrm{CD} 4^{+}$and $\mathrm{CD} 8^{+} \mathrm{T}$ cells [46]. In our study, we found that human PD-L1 gene in various lung cancer tissues and cell lines produced a non-coding isoform, NR_052005.1 
or PD-L1-lnc, which missed 106nt in exon 4 and 67 nt between exon 5 and 6 by alternative splicing (Fig. 3A). Since this isoform lacks an alternate internal segment and uses the 5'-most supported translational start codon as used in mRNA, it may serve as a candidate for nonsense-mediated mRNA decay (NMD) in protecting the mRNA degradation (https://www.ncbi.nlm.nih.gov/gene/29126). However, we found no effect of this isoform on the levels of PD-L1 mRNA and protein, instead, it acts as a functional lncRNA to promote lung adenocarcinoma progress via enhancing c-Myc activity (Fig. 9).

As a member of transcription factor MYC gene family, c-Myc plays a critical role in the development of human tumors and its overexpression has been detected in lung cancer of different histologic subtypes [47]. Previous study by Tang et al. [48] showed that a long non-coding RNA, LncRNA-GLCC1, could stabilize c-Myc thereby attenuating c-Myc's ubiquitination with the consequent promotion of colorectal carcinogenesis. Different from their study in which LncRNA-GLCC1 protected c-Myc from ubiquitination and degradation through interacting with HSP90 chaperon, our data suggest that PD-L1-lnc may directly interact with c-Myc. Crossimmunoprecipitation assays clearly showed the association of PD-L1-lnc with c-Myc (Fig. 6D-E). In line with the notion that PD-L1-lnc enhances c-Myc translocation into the nucleus and thus increases its transcriptional activity, we found that PD-L1-lnc overexpression increased the expression of a panel of c-Myc-modulated genes including IFITM1/3, MX1/2, IFI6 and SAA4, etc. Conversely, PD-L1-lnc depletion significantly decreased the expression of these genes.

The interactions between PD-L1-lnc with c-Myc were further validated by bioinformatics analysis using catRAPID omics [31] and RPISeq algorithms [32]. The results suggested that PD-L1-lnc could form hairpin structures, leading to a partial double helix structure. Multiple amino acids of c-Myc could form hydrogen bonds with their corresponding nucleobases in the double helix structure of PD-L1-lnc, which in turn, promotes c-Myc to form a basic helix-loop-helix Zip motif (Fig. 7A), leading to formation of more c-Myc-Max heterodimer and higher c-Myc transcriptional activity. 
We have also explored the potential mechanism that modulates alternative splicing for PD-L1-lnc generation. Given that the sequences of splice site in exon 4 and between exon 5 and 6 are UGGUAA, AGGAGA /AGGGAG, respectively. We blasted these sequences in Tomtom database [49] and found that the sequences of splice sites matched to the motifs recognized by three alternative splicing regulators, MSI1, DAZAP1 and ESRP2 (fig. S11A). DAZAP1 was selected to further study since the expression levels of MSI1 and ESRP2 in A549 cells were too low to have function (fig. S11B). To test whether DAZAP1 was involved in the generation of PD-L1-lnc, we checked the expression level of PD-L1-lnc in the A549 cells after transfection the specific siRNAs to DAZAP1. As shown in fig. S11C, the expression level of PD-L1-lnc was significantly decreased (right) after the downregulation of DAZAP1 caused by siRNAs (left), arguing that DAZAP1 may control the generation of PD-L1-lnc. However, further study is required to clarify whether and how DAZAP1 modulate PD-L1-lnc biogenesis.

In summary, the present study reveals that PD-L1-lnc, an alternatively spliced product of the PD-L1 gene, promotes lung adenocarcinoma proliferation, metastasis and survival via enhancing c-Myc transcriptional activity, and provides targeting PD-L1-lnc-c-Myc axis as a novel therapeutic strategy to improve the efficacy of lung cancer therapy. 


\section{ACCESSION NUMBERS}

The data of Microarray analysis in this study can be viewed in GEO database (GSE153051).

\section{ACKNOWLEGEMENTS}

The authors thank Professor Jill Leslie Littrell (Georgia State University, Atlanta, GA) for critical reading and constructive discussion of the manuscript.

\section{FUNDING}

This work was supported by grants from the Ministry of Science and Technology of China (2018YFA0507100), National Nature Science Foundation of China (31801088, 31670917, 31770981), Natural Science Foundation of Jiangsu Province (BK20170076) and Fundamental Research Funds for the Central Universities (020814380095, 020814380082).

\section{CONFLICT OF INTERESTS}

None declared.

\section{AUTHOR CONTRIBUTIONS}

Ken Zen, Hongwei Liang and Tao Wang designed research; Shuang Qu, Zichen Jiao, Geng Lu, Bing Yao, Ting Wang, Weiwei Rong, Jiahan Xu, Ting Fan, Xinlei Sun, and Rong Yang performed research and analyzed data; Jun Wang, Yongzhong Yao, Guifang Xu, Xin Yan, and Tao Wang collected the patients' samples; Ken Zen and Hongwei Liang wrote the paper. 


\section{References}

1. Siegel RL, Miller KD, Jemal A: Cancer statistics, 2018. CA Cancer J Clin 2018, 68:7-30.

2. Freeman GJ, Long AJ, Iwai Y, Bourque K, Chernova T, Nishimura H, Fitz LJ, Malenkovich N, Okazaki T, Byrne MC, et al: Engagement of the PD-1 immunoinhibitory receptor by a novel B7 family member leads to negative regulation of lymphocyte activation. J Exp Med 2000, 192:1027-1034.

3. Dong H, Strome SE, Salomao DR, Tamura H, Hirano F, Flies DB, Roche PC, Lu J, Zhu G, Tamada K, et al: Tumorassociated B7-H1 promotes T-cell apoptosis: a potential mechanism of immune evasion. Nat Med 2002, 8:793-800.

4. Robert C, Ribas A, Schachter J, Arance A, Grob JJ, Mortier L, Daud A, Carlino MS, McNeil CM, Lotem M, et al: Pembrolizumab versus ipilimumab in advanced melanoma (KEYNOTE-006): post-hoc 5-year results from an open-label, multicentre, randomised, controlled, phase 3 study. Lancet Oncol 2019, 20:1239-1251.

5. Berger R, Rotem-Yehudar R, Slama G, Landes S, Kneller A, Leiba M, Koren-Michowitz M, Shimoni A, Nagler A: Phase I safety and pharmacokinetic study of CT-011, a humanized antibody interacting with PD-1, in patients with advanced hematologic malignancies. Clin Cancer Res 2008, 14:3044-3051.

6. Hellmann MD, Kim TW, Lee CB, Goh BC, Miller WH, Oh DY, Jamal R, Chee CE, Chow LQM, Gainor JF, et al: Phase Ib study of atezolizumab combined with cobimetinib in patients with solid tumors. Annals Of Oncology 2019, 30:1134-1142.

7. Powles T, Eder JP, Fine GD, Braiteh FS, Loriot Y, Cruz C, Bellmunt J, Burris HA, Petrylak DP, Teng SL, et al: MPDL3280A (anti-PD-L1) treatment leads to clinical activity in metastatic bladder cancer. Nature 2014, 515:558-+,

8. Topalian SL, Hodi FS, Brahmer JR, Gettinger SN, Smith DC, McDermott DF, Powderly JD, Carvajal RD, Sosman JA, Atkins MB, et al: Safety, Activity, and Immune Correlates of Anti-PD-1 Antibody in Cancer. New England Journal Of Medicine 2012, 366:2443-2454.

9. Herbst RS, Soria JC, Kowanetz M, Fine GD, Hamid O, Gordon MS, Sosman JA, McDermott DF, Powderly JD, Gettinger SN, et al: Predictive correlates of response to the anti-PD-L1 antibody MPDL3280A in cancer patients. Nature 2014, 515:563-+.

10. Cerezo M, Guemiri R, Druillennec S, Girault I, Malka-Mahieu H, Shen SS, Allard D, Martineau S, Welsch C, Agoussi $\mathrm{S}$, et al: Translational control of tumor immune escape via the elF4F-STAT1-PD-L1 axis in melanoma. Nature Medicine 2018, 24:1877-+.

11. He XH, Xu LH, Liu Y: Identification of a novel splice variant of human PD-L1 mRNA encoding an isoform-lacking Igv-like domain. Acta Pharmacol Sin 2005, 26:462-468.

12. Faas SJ, Giannoni MA, Mickle AP, Kiesecker CL, Reed DJ, Wu D, Fodor WL, Mueller JP, Matis LA, Rother RP: Primary structure and functional characterization of a soluble, alternatively spliced form of B7-1. $J$ Immunol 2000, 164:6340-6348.

13. Galon J, Bruni D: Approaches to treat immune hot, altered and cold tumours with combination immunotherapies. Nat Rev Drug Discov 2019, 18:197-218.

14. Zhang Y, Chen L: Classification of Advanced Human Cancers Based on Tumor Immunity in the MicroEnvironment (TIME) for Cancer Immunotherapy. JAMA Oncol 2016, 2:1403-1404.

15. Ribas A, Dummer R, Puzanov I, VanderWalde A, Andtbacka RHI, Michielin O, Olszanski AJ, Malvehy J, Cebon J, Fernandez E, et al: Oncolytic Virotherapy Promotes Intratumoral T Cell Infiltration and Improves Anti-PD-1 Immunotherapy. Cell 2018, 174:1031-1032.

16. Dhamija S, Diederichs S: From junk to master regulators of invasion: IncRNA functions in migration, EMT and metastasis. Int J Cancer 2016, 139:269-280. 
17. Batista PJ, Chang HY: Long noncoding RNAs: cellular address codes in development and disease. Cell 2013, 152:1298-1307.

18. Grelet S, Link LA, Howley B, Obellianne C, Palanisamy V, Gangaraju VK, Diehl JA, Howe PH: A regulated PNUTS mRNA to IncRNA splice switch mediates EMT and tumour progression. Nat Cell Biol 2017, 19:1105-1115.

19. Wang ET, Sandberg R, Luo S, Khrebtukova I, Zhang L, Mayr C, Kingsmore SF, Schroth GP, Burge CB: Alternative isoform regulation in human tissue transcriptomes. Nature 2008, 456:470-476.

20. Costa FF: Non-coding RNAs: new players in eukaryotic biology. Gene 2005, 357:83-94.

21. Ji M, Liu Y, Li Q, Li X, Ning Z, Zhao W, Shi H, Jiang J, Wu C: PD-1/PD-L1 expression in non-small-cell lung cancer and its correlation with EGFR/KRAS mutations. Cancer Biol Ther 2016, 17:407-413.

22. Zhang $M$, Li G, Wang $Y$, Wang $Y$, Zhao $S$, Haihong $P$, Zhao $H$, Wang Y: PD-L1 expression in lung cancer and its correlation with driver mutations: a meta-analysis. Sci Rep 2017, 7:10255.

23. Baker AM, Huang W, Wang XM, Jansen M, Ma XJ, Kim J, Anderson CM, Wu X, Pan L, Su N, et al: Robust RNAbased in situ mutation detection delineates colorectal cancer subclonal evolution. Nat Commun 2017, 8:1998.

24. Ayers M, Lunceford J, Nebozhyn M, Murphy E, Loboda A, Kaufman DR, Albright A, Cheng JD, Kang SP, Shankaran V, et al: IFN-gamma-related mRNA profile predicts clinical response to PD-1 blockade. J Clin Invest 2017, 127:2930-2940.

25. Garcia-Diaz A, Shin DS, Moreno BH, Saco J, Escuin-Ordinas H, Rodriguez GA, Zaretsky JM, Sun L, Hugo W, Wang $X$, et al: Interferon Receptor Signaling Pathways Regulating PD-L1 and PD-L2 Expression. Cell Rep 2017, 19:1189-1201.

26. Karousis ED, Muhlemann O: Nonsense-Mediated mRNA Decay Begins Where Translation Ends. Cold Spring Harb Perspect Biol 2019, 11.

27. Diermeier-Daucher S, Clarke ST, Hill D, Vollmann-Zwerenz A, Bradford JA, Brockhoff G: Cell type specific applicability of 5-ethynyl-2'-deoxyuridine (EdU) for dynamic proliferation assessment in flow cytometry. Cytometry A 2009, 75:535-546.

28. Justus CR, Leffler N, Ruiz-Echevarria M, Yang LV: In vitro cell migration and invasion assays. J Vis Exp 2014.

29. Rieger AM, Nelson KL, Konowalchuk JD, Barreda DR: Modified annexin V/propidium iodide apoptosis assay for accurate assessment of cell death. J Vis Exp 2011.

30. Jung M, Russell AJ, Liu B, George J, Liu PY, Liu T, DeFazio A, Bowtell DD, Oberthuer A, London WB, et al: A Myc Activity Signature Predicts Poor Clinical Outcomes in Myc-Associated Cancers. Cancer Res 2017, 77:971-981.

31. Agostini F, Zanzoni A, Klus P, Marchese D, Cirillo D, Tartaglia GG: catRAPID omics: a web server for large-scale prediction of protein-RNA interactions. Bioinformatics 2013, 29:2928-2930.

32. Muppirala UK, Honavar VG, Dobbs D: Predicting RNA-protein interactions using only sequence information. BMC Bioinformatics 2011, 12:489.

33. Zuker M: Mfold web server for nucleic acid folding and hybridization prediction. Nucleic Acids Res 2003, 31:3406-3415.

34. Popenda M, Szachniuk M, Antczak M, Purzycka KJ, Lukasiak P, Bartol N, Blazewicz J, Adamiak RW: Automated 3D structure composition for large RNAs. Nucleic Acids Res 2012, 40:e112.

35. Tuszynska I, Magnus M, Jonak K, Dawson W, Bujnicki JM: NPDock: a web server for protein-nucleic acid docking. Nucleic Acids Res 2015, 43:W425-430.

36. Sammak S, Hamdani N, Gorrec F, Allen MD, Freund SMV, Bycroft M, Zinzalla G: Crystal Structures and Nuclear Magnetic Resonance Studies of the Apo Form of the c-MYC:MAX bHLHZip Complex Reveal a Helical Basic Region in the Absence of DNA. Biochemistry 2019, 58:3144-3154.

37. Chen DS, Mellman I: Oncology meets immunology: the cancer-immunity cycle. Immunity 2013, 39:1-10.

38. Keir ME, Butte MJ, Freeman GJ, Sharpe AH: PD-1 and its ligands in tolerance and immunity. Annu Rev Immunol 


\section{8, 26:677-704.}

39. Okamura H, Tsutsi H, Komatsu T, Yutsudo M, Hakura A, Tanimoto T, Torigoe K, Okura T, Nukada Y, Hattori K, et al.: Cloning of a new cytokine that induces IFN-gamma production by T cells. Nature 1995, 378:88-91.

40. Tannenbaum CS, Hamilton TA: Immune-inflammatory mechanisms in IFNgamma-mediated anti-tumor activity. Semin Cancer Biol 2000, 10:113-123.

41. Corthay A, Skovseth DK, Lundin KU, Rosjo E, Omholt H, Hofgaard PO, Haraldsen G, Bogen B: Primary antitumor immune response mediated by CD4+ T cells. Immunity 2005, 22:371-383.

42. Zaidi MR, Merlino G: The two faces of interferon-gamma in cancer. Clin Cancer Res 2011, 17:6118-6124.

43. Ubil E, Caskey L, Holtzhausen A, Hunter D, Story C, Earp HS: Tumor-secreted Pros1 inhibits macrophage M1 polarization to reduce antitumor immune response. J Clin Invest 2018, 128:2356-2369.

44. Chen G, Huang AC, Zhang W, Zhang G, Wu M, Xu W, Yu Z, Yang J, Wang B, Sun H, et al: Exosomal PD-L1 contributes to immunosuppression and is associated with anti-PD-1 response. Nature 2018, 560:382-386.

45. Hassounah NB, Malladi VS, Huang Y, Freeman SS, Beauchamp EM, Koyama S, Souders N, Martin S, Dranoff G, Wong KK, et al: Identification and characterization of an alternative cancer-derived PD-L1 splice variant. Cancer Immunol Immunother 2019, 68:407-420.

46. Zhou J, Mahoney KM, Giobbie-Hurder A, Zhao FM, Lee S, Liao XY, Rodig S, Li JJ, Wu XQ, Butterfield LH, et al: Soluble PD-L1 as a Biomarker in Malignant Melanoma Treated with Checkpoint Blockade. Cancer Immunology Research 2017, 5:480-492.

47. Zajac-Kaye M: Myc oncogene: a key component in cell cycle regulation and its implication for lung cancer. Lung Cancer 2001, 34 Suppl 2:S43-46.

48. Tang J, Yan T, Bao Y, Shen C, Yu C, Zhu X, Tian X, Guo F, Liang Q, Liu Q, et al: LncRNA GLCC1 promotes colorectal carcinogenesis and glucose metabolism by stabilizing c-Myc. Nat Commun 2019, 10:3499.

49. Gupta S, Stamatoyannopoulos JA, Bailey TL, Noble WS: Quantifying similarity between motifs. Genome Biology 2007,8 . 
A

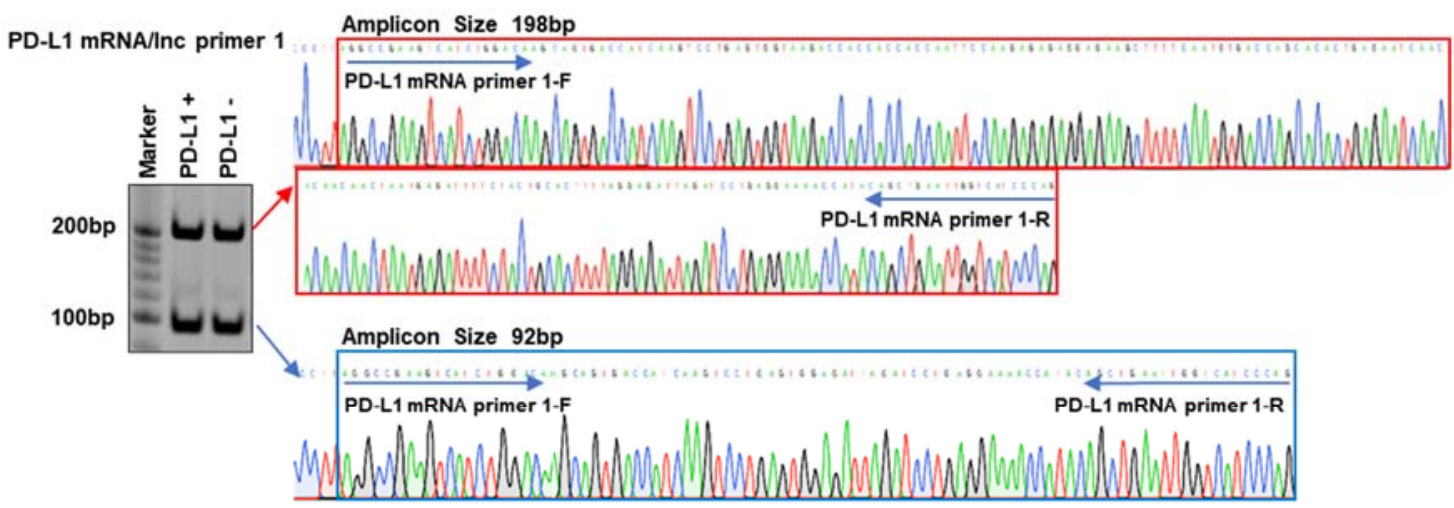

B

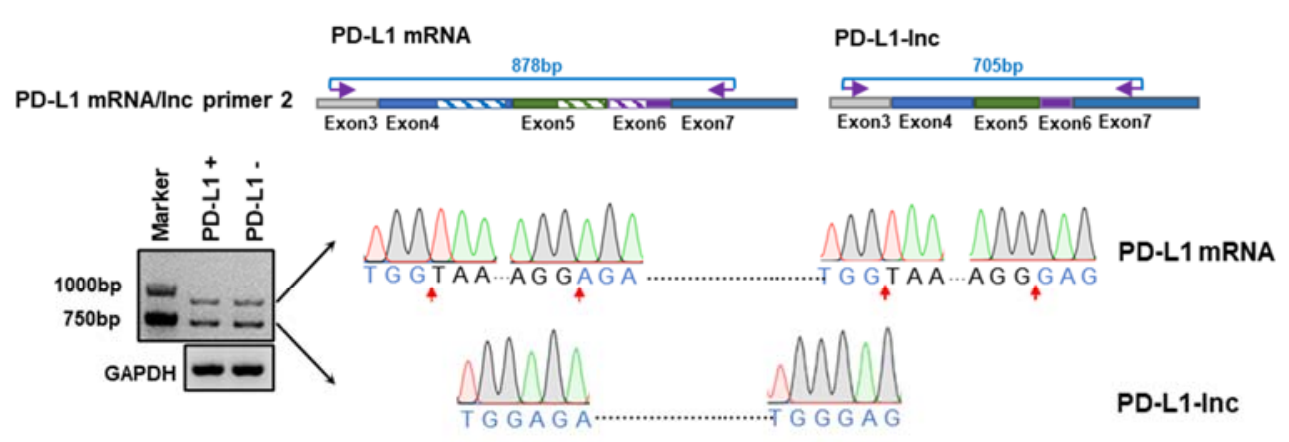

C

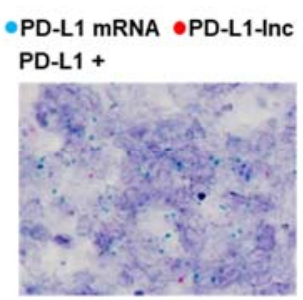

D

PD-L1 -

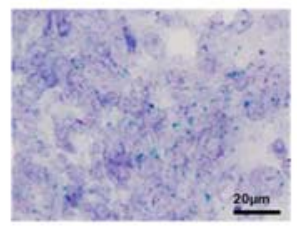

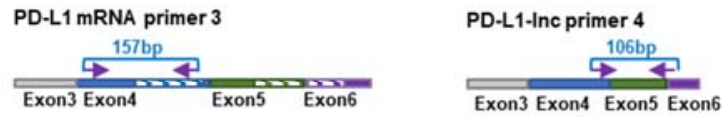
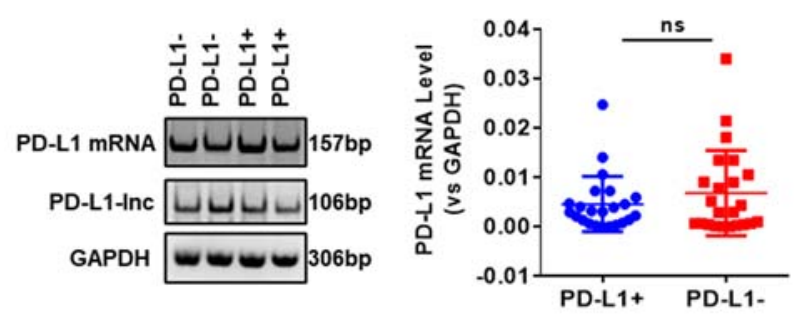

Figure 1. Generation of PD-L1-Inc in PD-L1 protein positive or negative lung adenocarcinoma via alternative splicing. (A) Agarose gel analysis and Sanger sequencing of the PCR end-product in PD-L1 protein positive or negative tumor tissues. (B) Upper, the schematic of primers for amplification of PD-L1 mRNA and PD-L1-lnc; Lower, the agarose gel and Sanger sequencing of the 878bp and 705bp band obtained by RT-PCR. (C) RNA BaseScope for PD-L1 mRNA (blue) and PD-L1-lnc (red) in PD-L1 protein positive and negative tumor tissues. (D) Upper, the schematic of primer for amplification of PD-L1 mRNA and PD-L1-lnc; Lower, the expression level of PD-L1 mRNA and PD-L1-lnc in PD-L1 protein-positive or -negative tumor tissues by qRT-PCR with specific probes. 
A

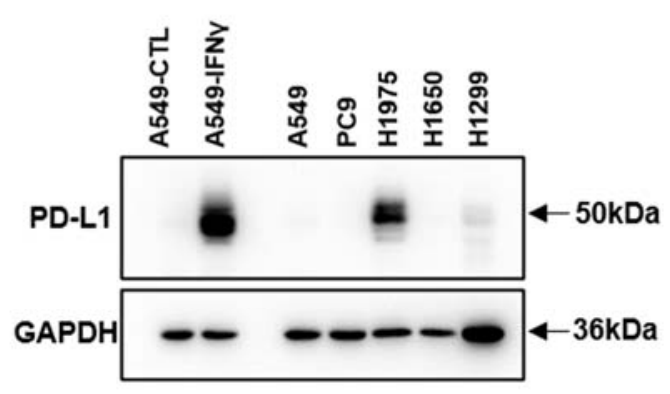

C

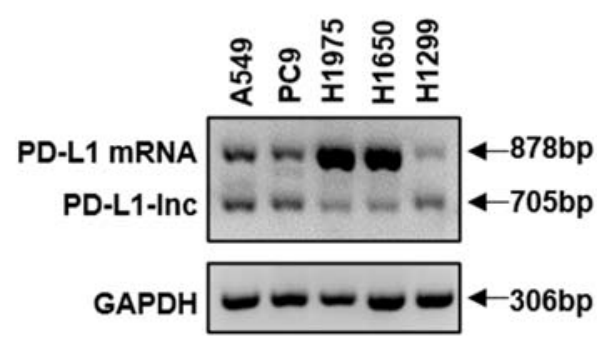

$\mathbf{E}$

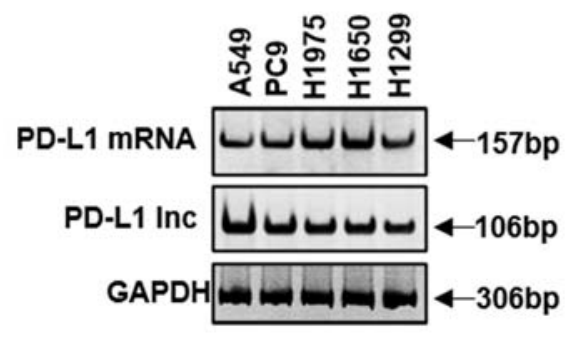

B
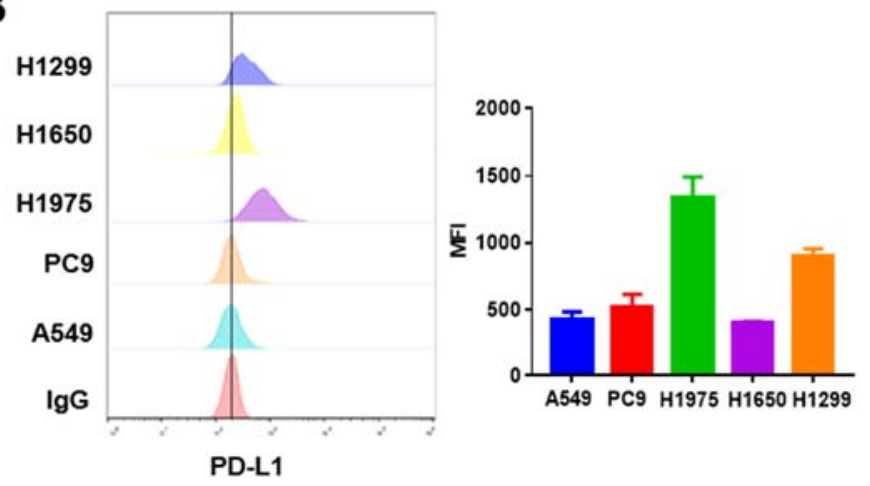

D
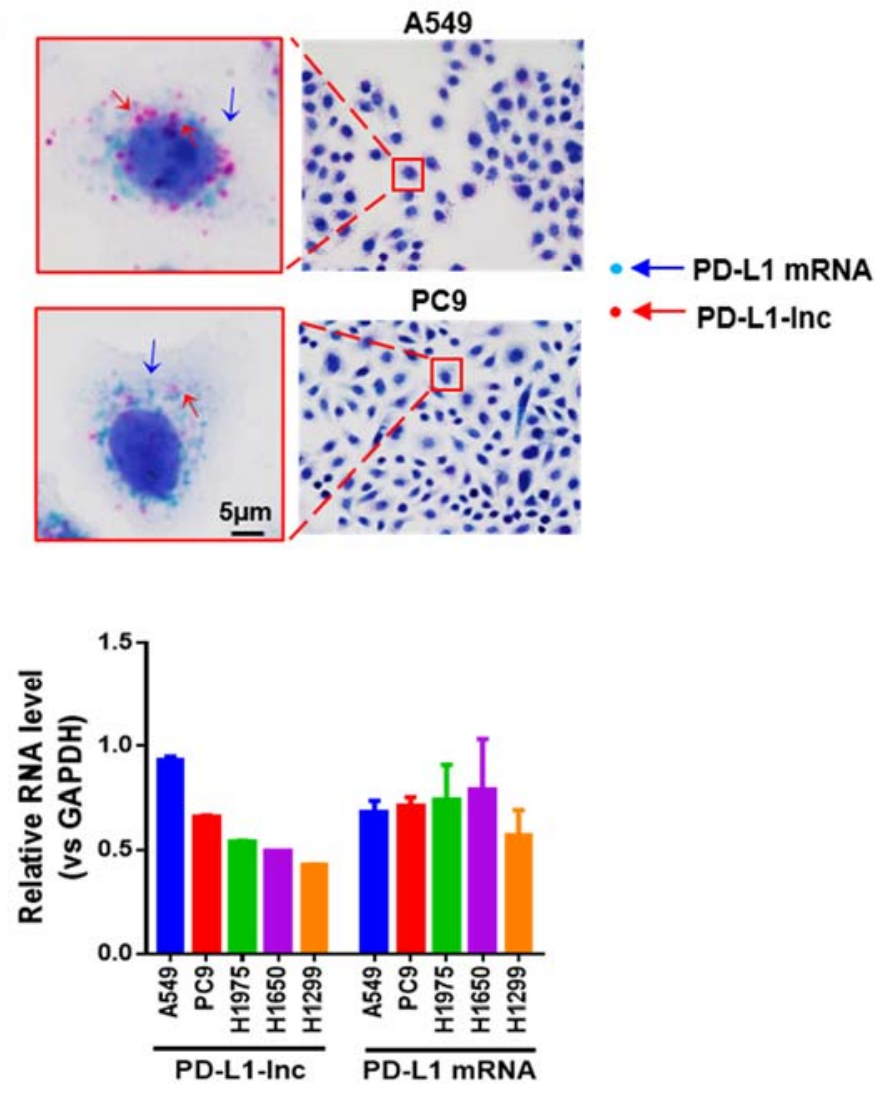

Figure 2. The expression of PD-L1-Inc in various lung adenocarcinoma cancer cell lines. (A-B) Western blotting (A) and flow cytometry (B) analysis of PD-L1 in various lung adenocarcinoma cancer cell lines. (C) Agarose gel analysis of PCR end-product of PD-L1 transcript showing two bands at 878bp and 705bp. (D) RNA BaseScope for PD-L1 mRNA (blue) and PD-L1-lnc (red) in A549 and PC9 cells. (E) The expression level of PD-L1 mRNA and PD-L1-lnc in various lung adenocarcinoma cancer cell lines detected by qRT-PCR with specific primers. 
A

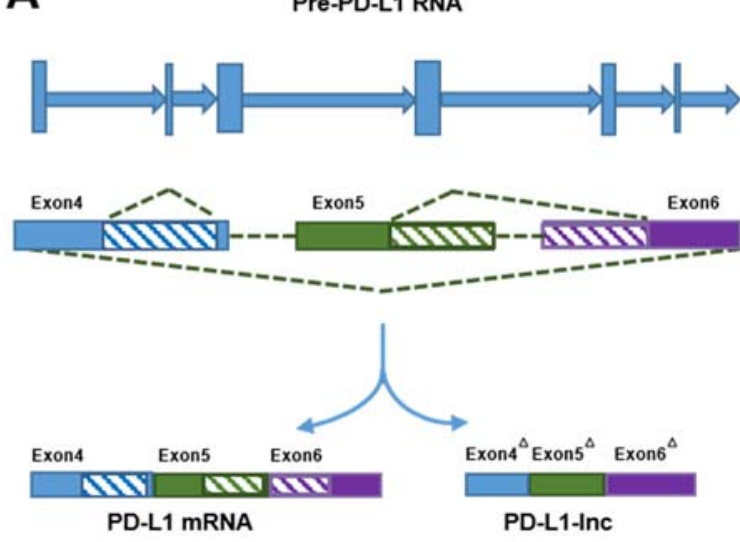

B
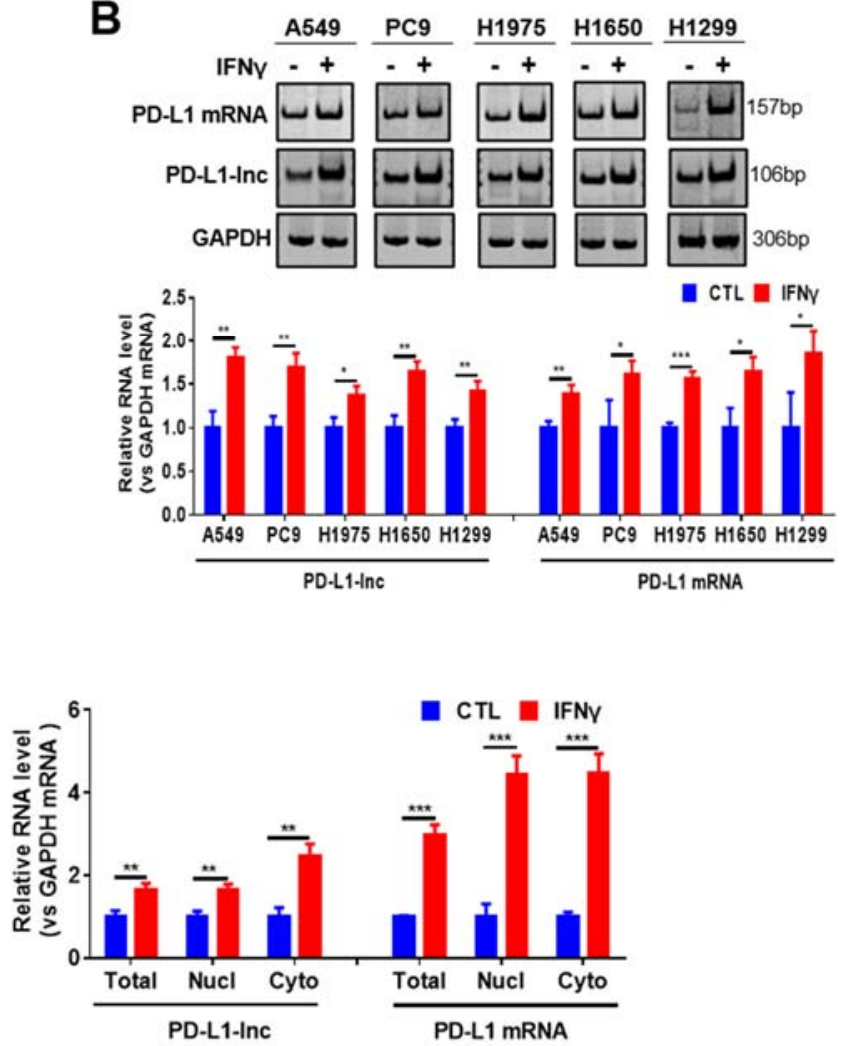

Figure 3. Human lung adenocarcinoma PD-L1-Inc was regulated by IFN $\gamma$ treatment. (A) Schematic representation of the alternative splice region of the PD-L1 variants. (B) Agarose gel analysis of the expression level of PD-L1 mRNA and lncRNA in lung adenocarcinoma cells with or without IFN $\gamma$ treatment. Upper: representative image; Lower: quantitative analysis. (C) Cellular distribution of PD-L1-lnc and PD-L1 mRNA in A549 cells with or without IFN $\gamma$ treatment. Left: representative image; right: quantitative analysis. $* P<0.05$; ${ }^{* *} P<0.01 ; * * * P<0.001$. 
A
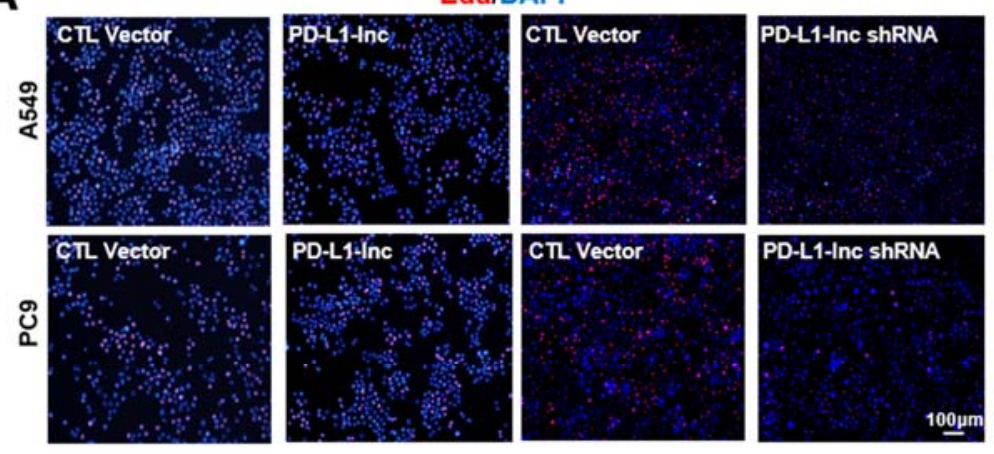

B
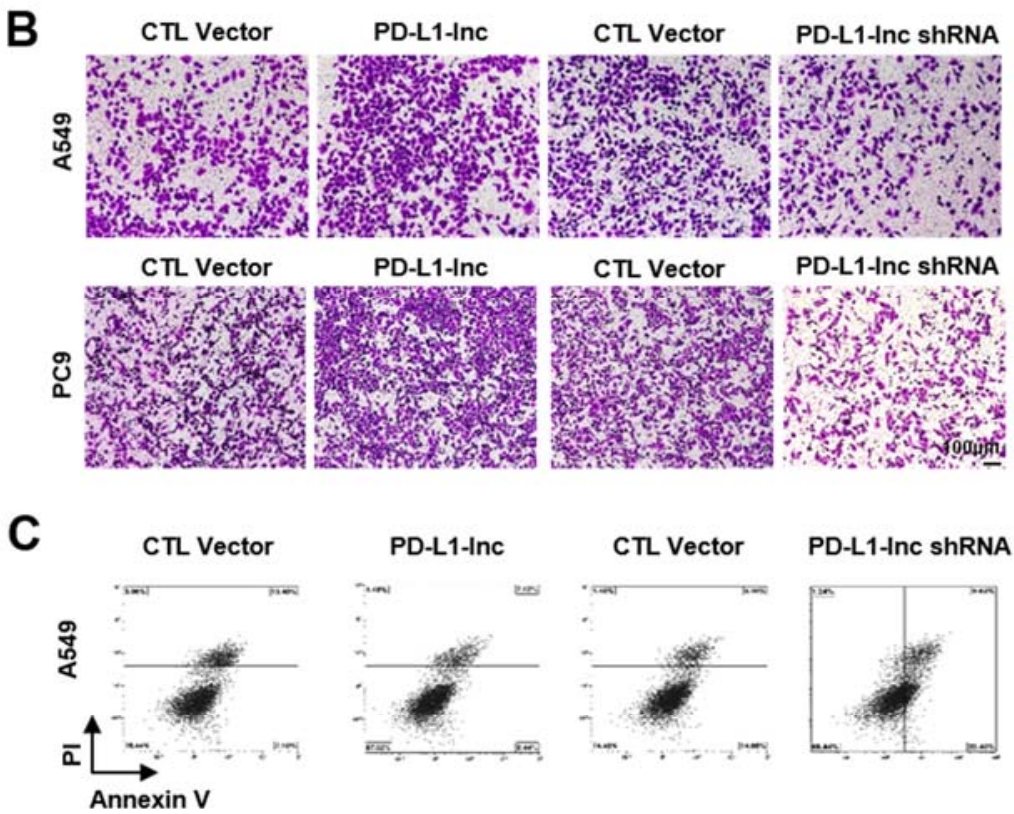

CTL Vector

PD-L1-Inc
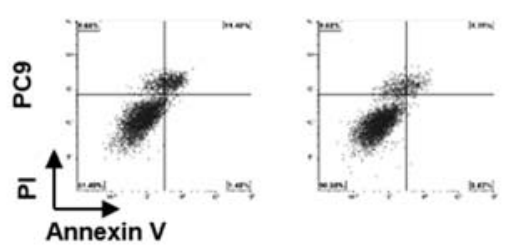

CTL Vector

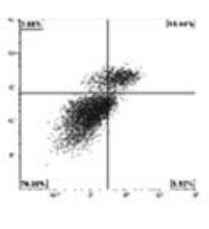

PD-L1-Inc ShRNA

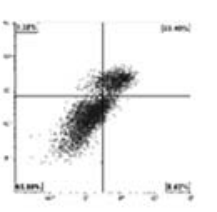

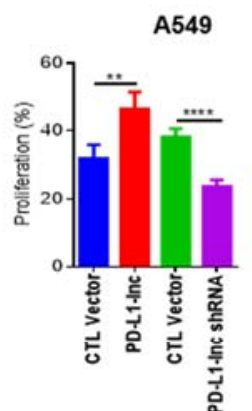
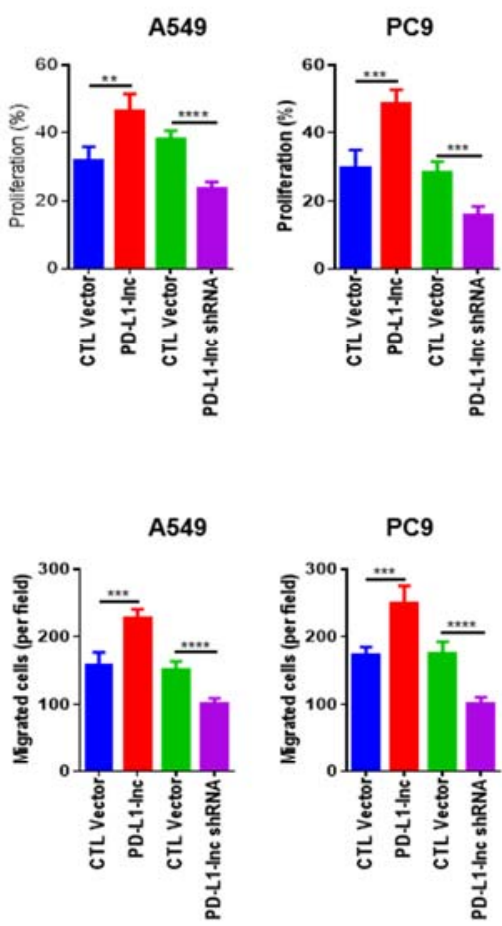

A549

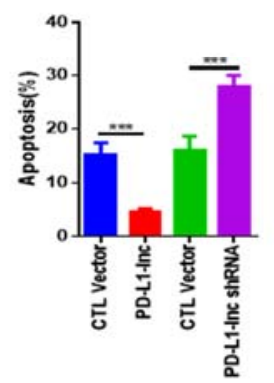

PC9

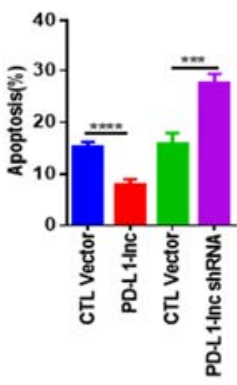

Figure 4. Effects of PD-L1-Inc on the proliferation, invasion and apoptosis of human lung cancer cells. A549 and PC9 cells were transfected with PD-L1-lnc-expressing vector or PD-L1-lnc shRNA-expressing vector. (A) The proliferation of A549 and PC9 cells detected by Edu assay. (B) The invasion of A549 and PC9 cells detected by Transwell assay. (C) The apoptosis of A549 and PC9 cells detected by flow cytometry. In ac, left: representative image; right: quantitative analysis. ${ }^{* *} P<0.01 ; * * * P<0.001 ; * * * * P<0.0001$. 
A

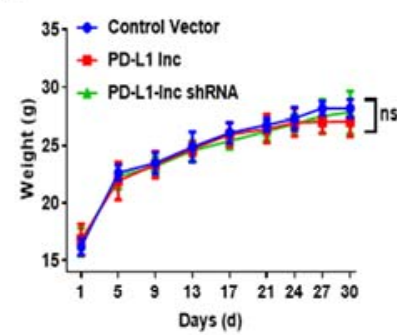

D
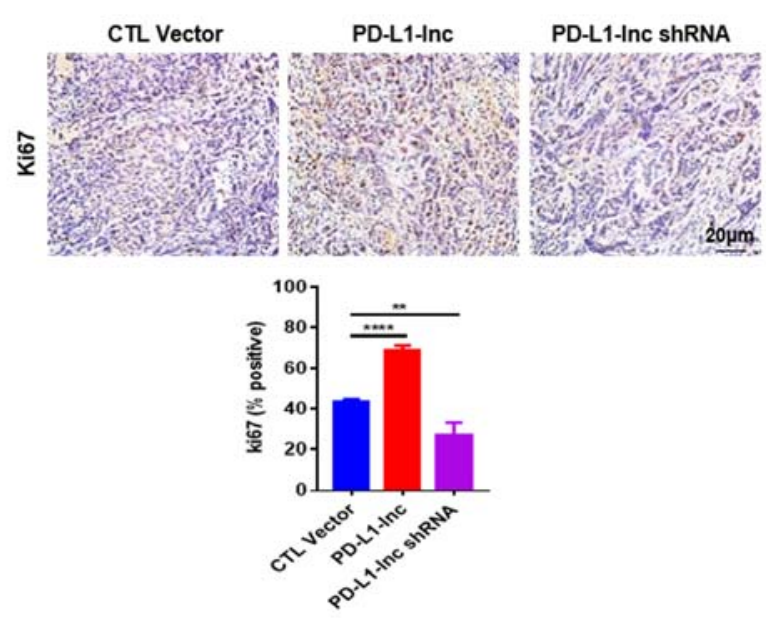

C

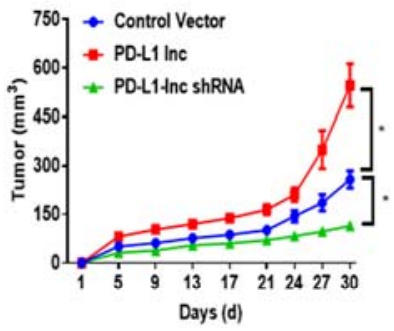

E

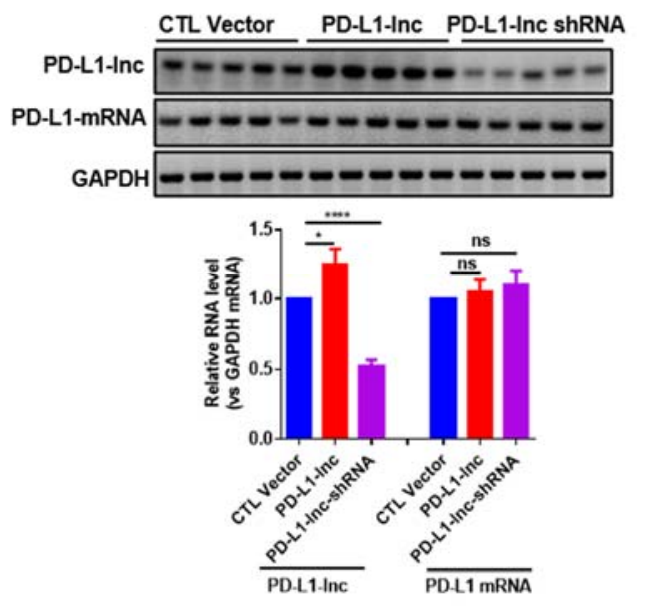

Figure 5. Effects of PD-L1-Inc on the growth of lung cancer cell xenografts in mice. (A) The growth rate of mouse weight during the timeframe of experiment. (B) The growth rate of various tumor cells implanted in the mice. (C) The weight of various implanted tumors on 30 days post-injection. (D) Proliferation of implanted tumors in the mice. Upper: representative immunohistochemical staining of Ki67 in implanted tumors; Lower: quantitative analysis. (E) qRT-PCR analysis of the expression levels of PD-L1-lnc in the implanted tumors. Upper: gel image of PCR product; Lower: qRT-PCR analysis. ns, no significance. ${ }^{*} P<0.05 ; * * P<0.01$. 
A

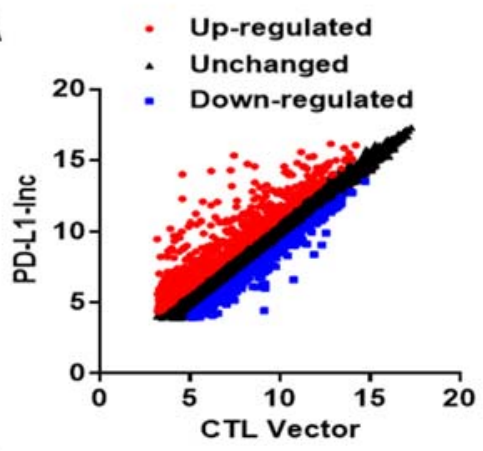

C

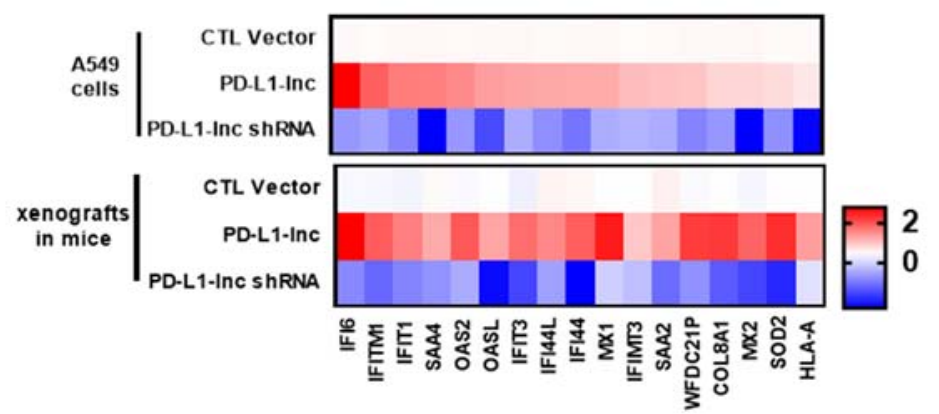

D

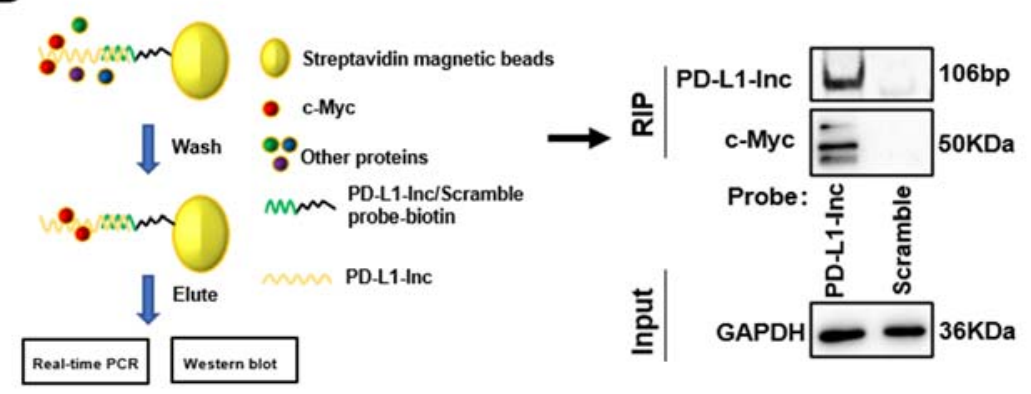

E

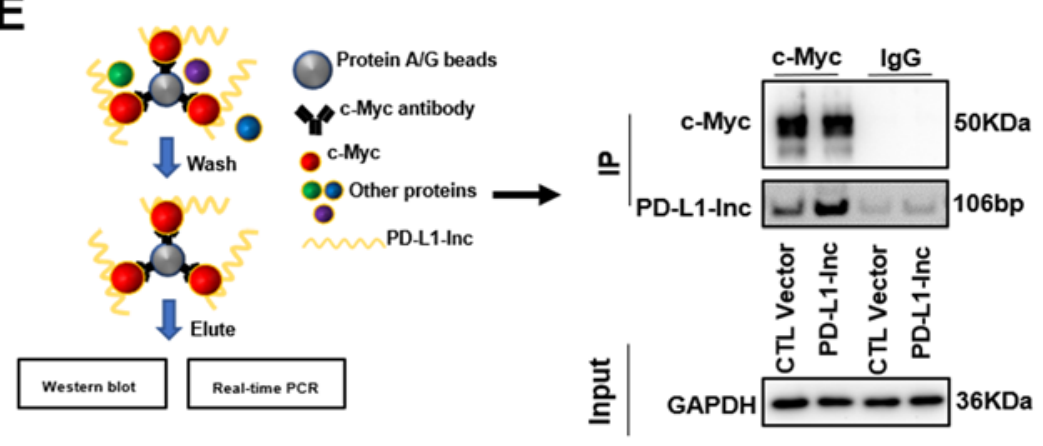

B

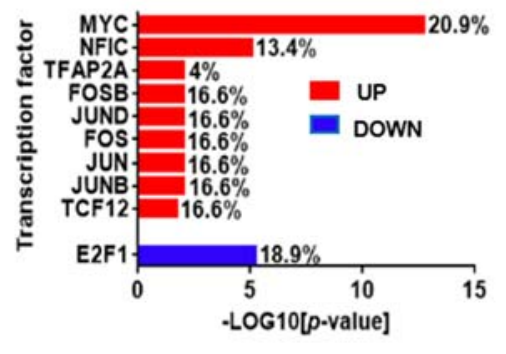


A
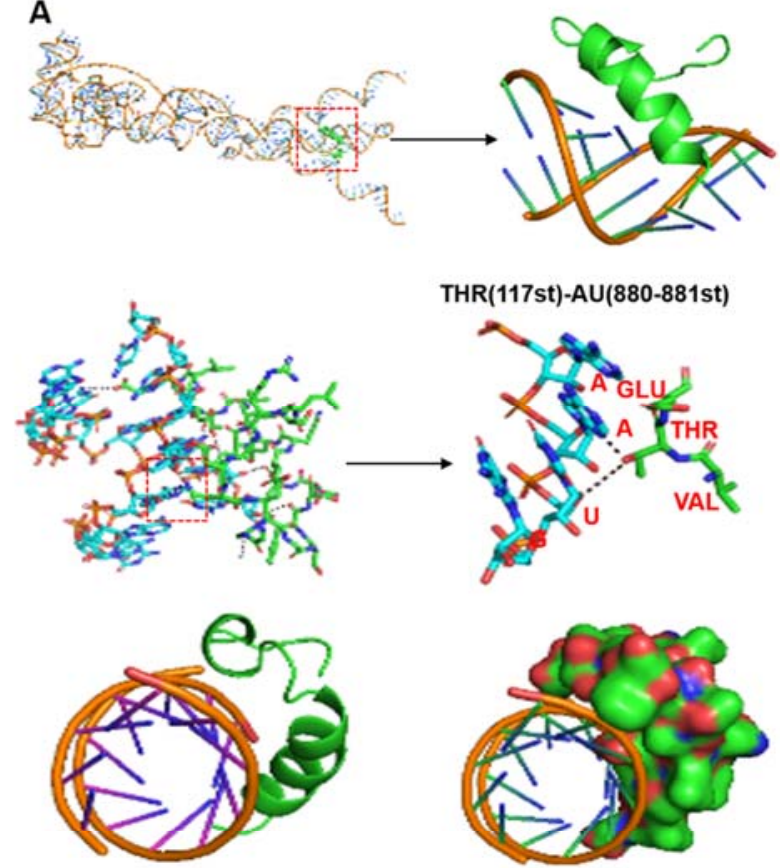

E

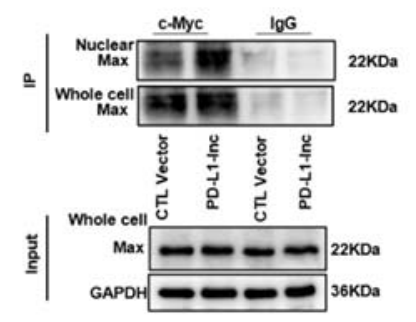

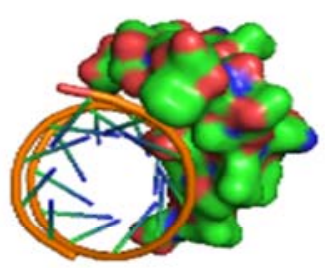

$\mathbf{F}$

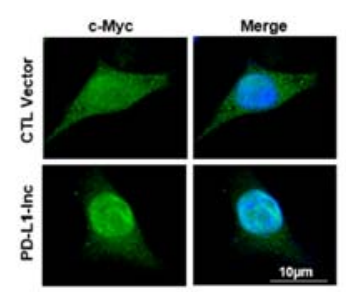

B

C $\begin{array}{ccc}870 & 190 & 990 \\ \text { PD-L1-Inc GATGCAGGCAATGTGGGACTIAAAAGGCCCAAGCACTGAAA } & 10 \\ \text { PD-L1-Inc mut GATGCAGCCATACTGGGACTTAAAAGGCCCATCCACTGAAA }\end{array}$

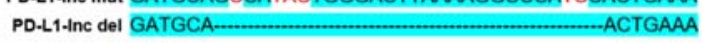

C D
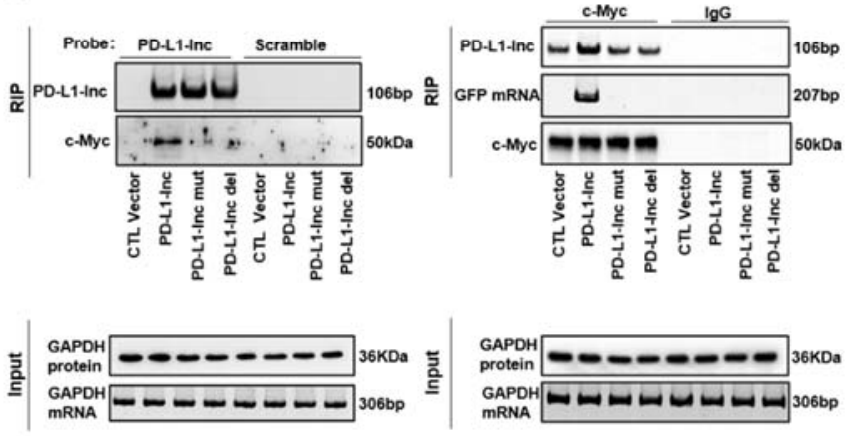

G
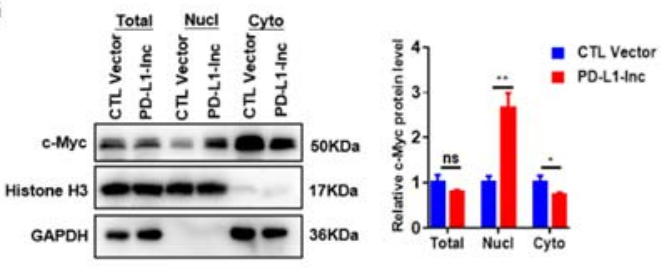

Figure 7. Binding of PD-L1-Inc with c-Myc enhances the association of c-Myc with MAX and promotes the entry of c-Myc into nucleus. (A) Graphical representation of three-dimensional structures of PD-L1-lnc and c-Myc docking models with a zoom-in image of the binding interface. The binding region was shown in three different visualizations (cartoon and sphere). (B) Schematic representation of the PD-L1-lnc-del vector which was deleted the predicted binding sites and the PD-L1-lnc-mut vector which was mutated at the predicted binding sites (marked red). (C) Pulldown of PD-L1-lnc in A549 cells transfected with WT or mutant PD-L1-lnc/GFP vector using biotinylated antisense probes against GFP mRNA to detect the binding of PDL1-lnc with c-Myc. (D) Immunoprecipitation of c-Myc in A549 cells transfected with WT or mutant PD-L1lnc/GFP vector using anti-c-Myc antibody to assess the binding of PD-L1-lnc with c-Myc. (E) Binding of PDL1-lnc with c-Myc resulted in recruitment of MAX by c-Myc in A549 cells. (F-G) Immunofluorescence (F) and cell fractionation/Western blot analysis $(\mathrm{G})$ of c-Myc distribution in A549 cells before and after PD-L1lnc overexpression. Note that PD-L1-lnc overexpression markedly increases the distribution of c-Myc in the nuclei or nuclear fraction (nucl) of A549 cells. 
A
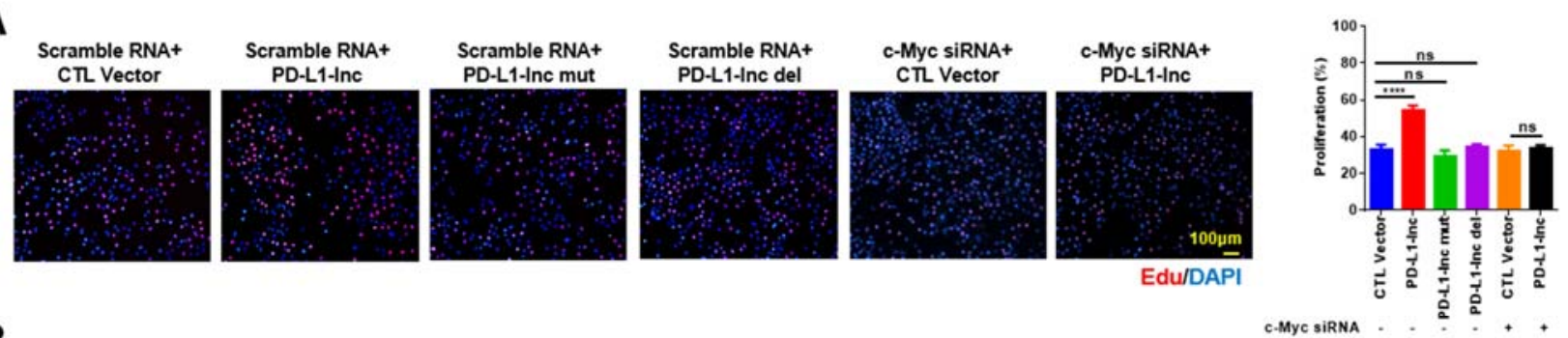

B
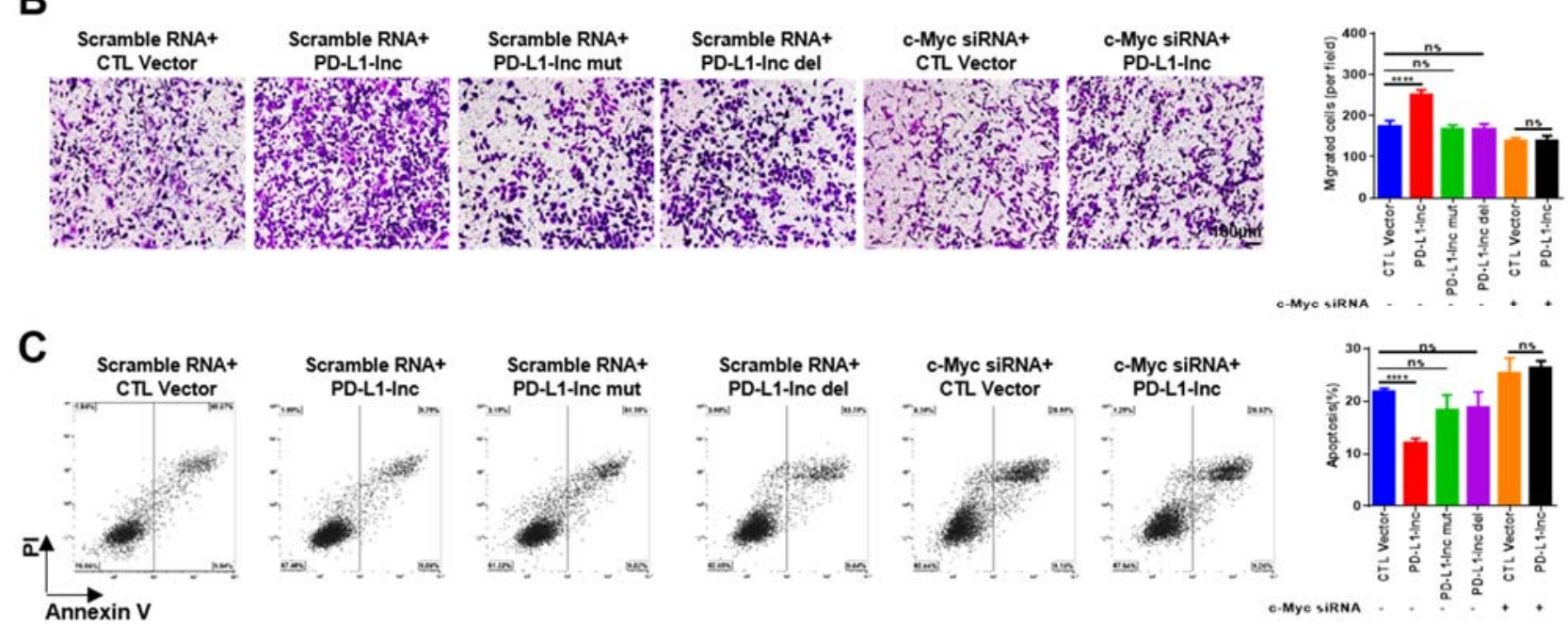

Figure 8. PD-L1-Inc promotes lung adenocarcinoma progression via c-Myc. (A-C) Overexpression of PDL1-lnc mutant or knockdown of c-Myc protein expression in A549 cells both abolished the effect of PD-L1lnc on A549 cell proliferation (A), migration (B) and resistance to apoptosis (C). In A-C, left panels, representative image from three independent experiments; right panels, quantitative analysis of images. $* \mathrm{p}<0.05 ; * \mathrm{p}<0.01$. 


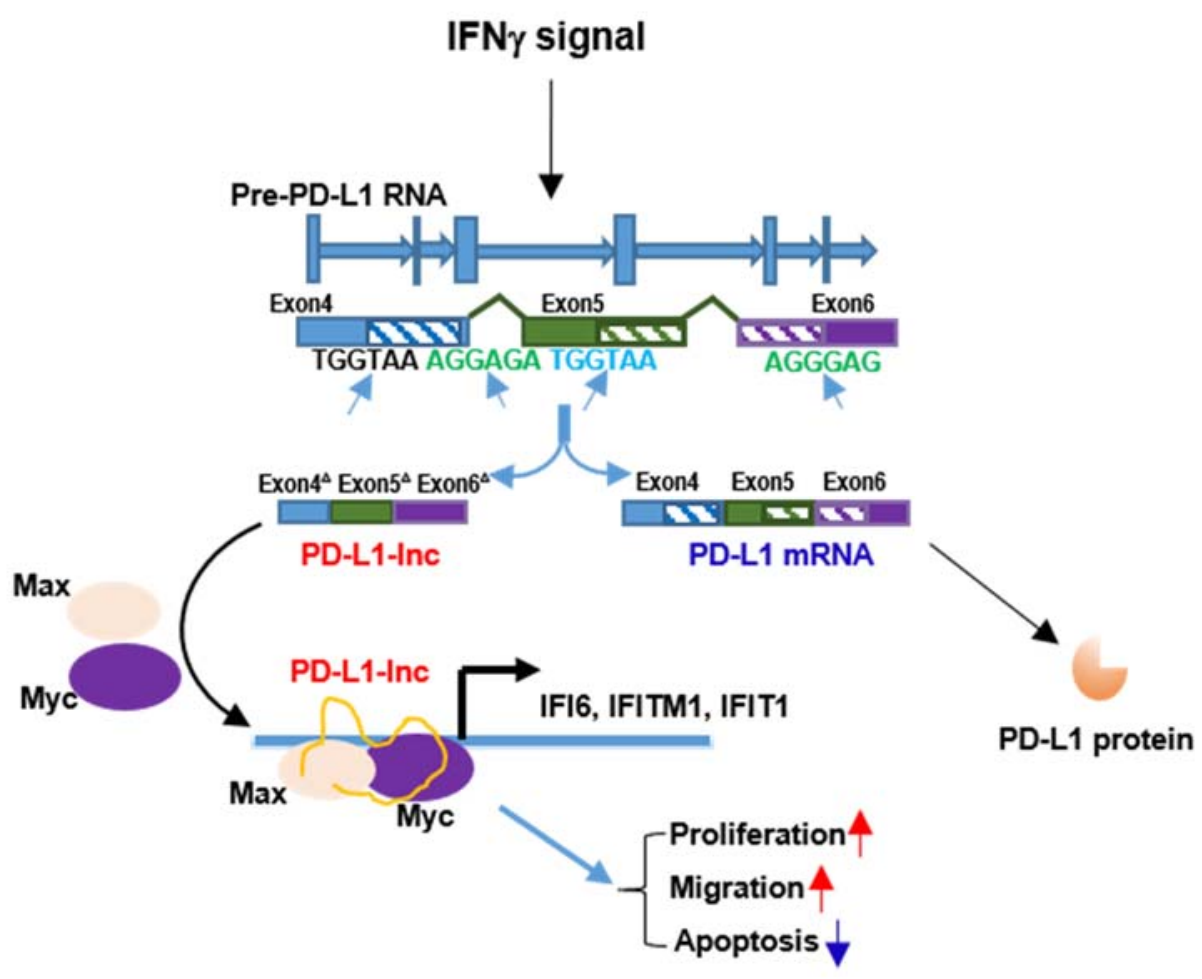

Figure 9. PD-L1 mRNA alternative splice (PD-L1-lnc) promotes lung adenocarcinoma progression via enhancing c-Myc activity. 
A

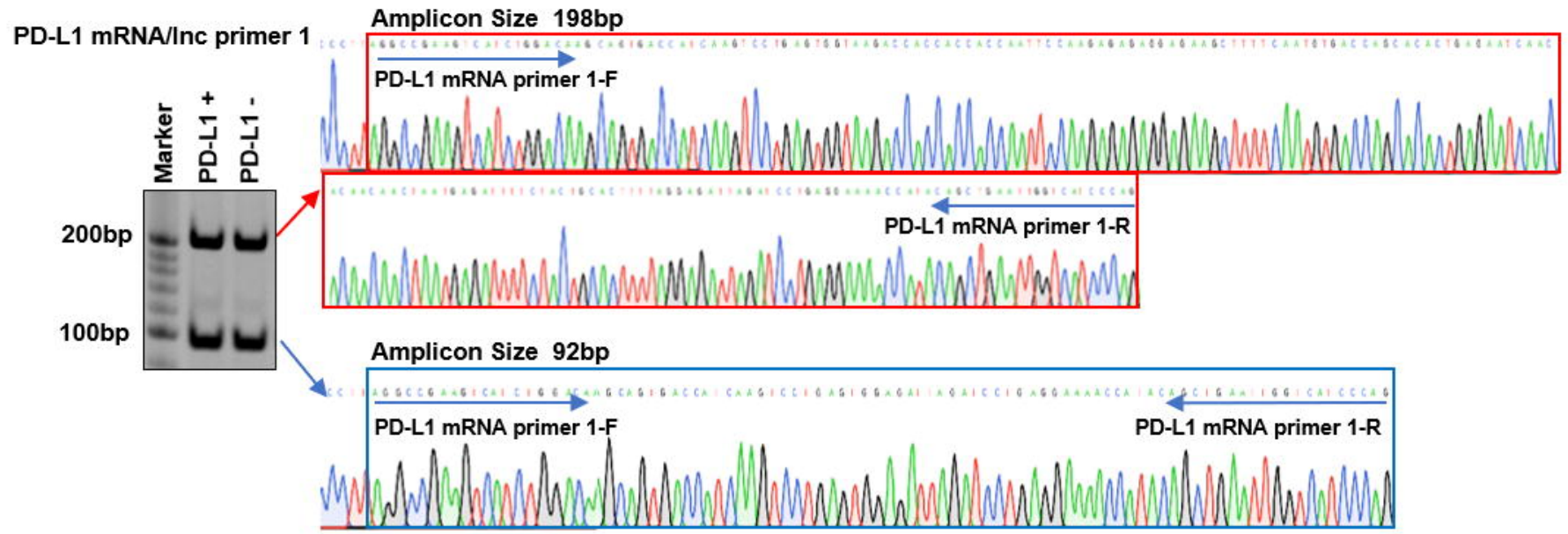

B

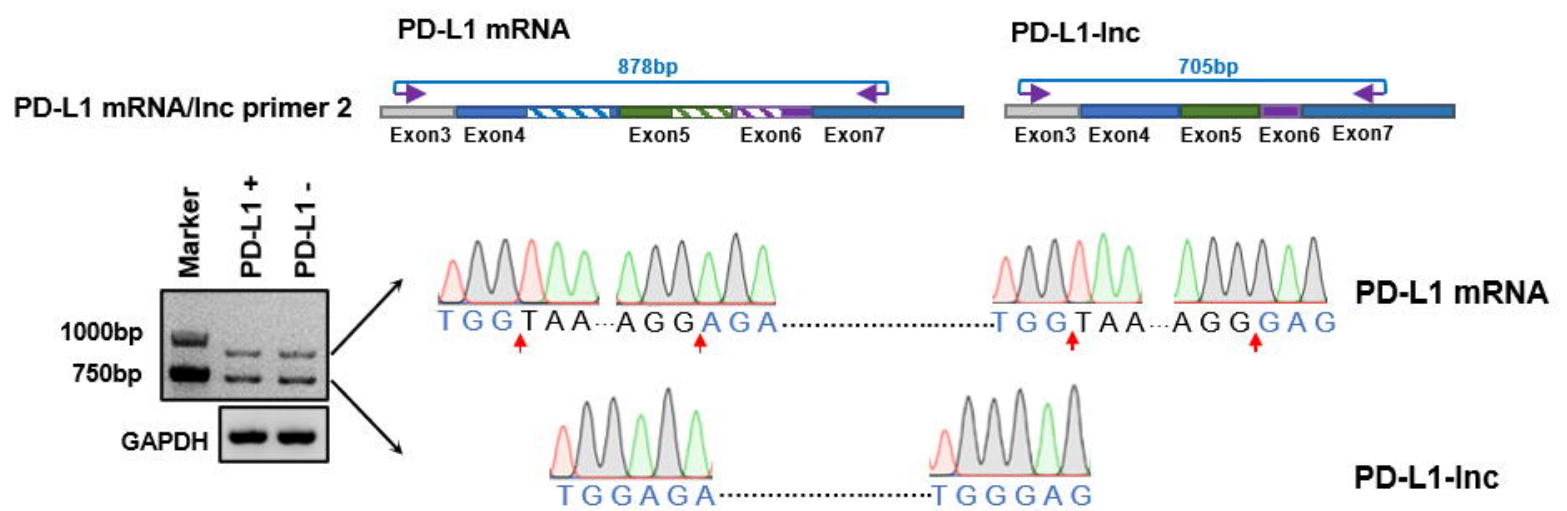

C

D

-PD-L1 mRNA •PD-L1-Inc PD-L1 +

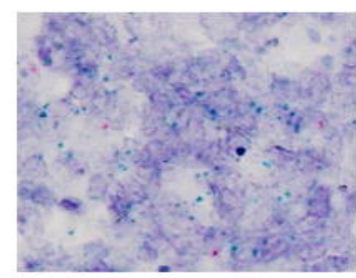

PD-L1 -

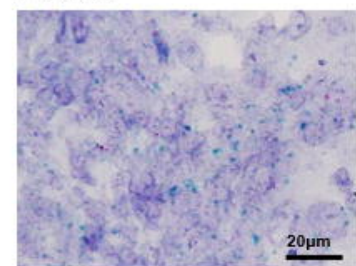

PD-L1 mRNA primer 3

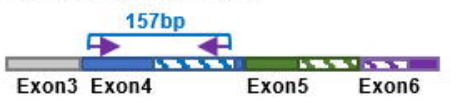

PD-L1 mRNA

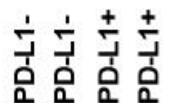

PD-L1 Inc

GAPDH ๒பேப157bp

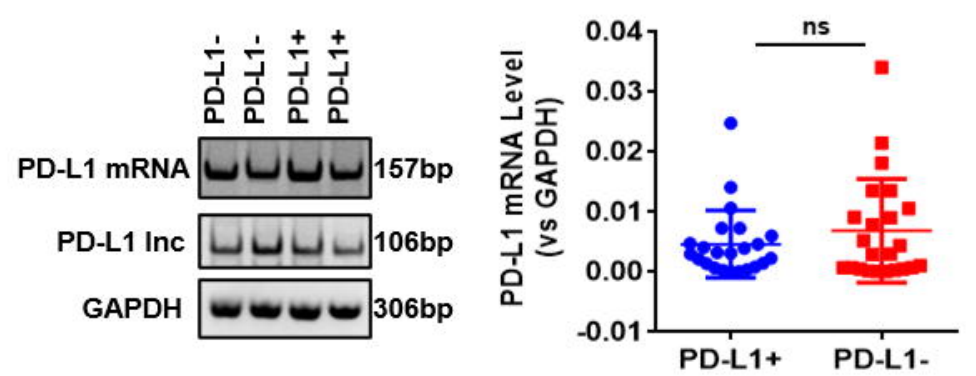

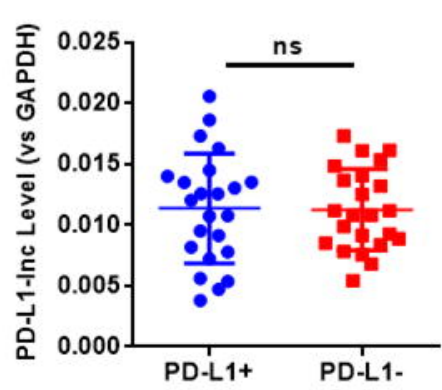

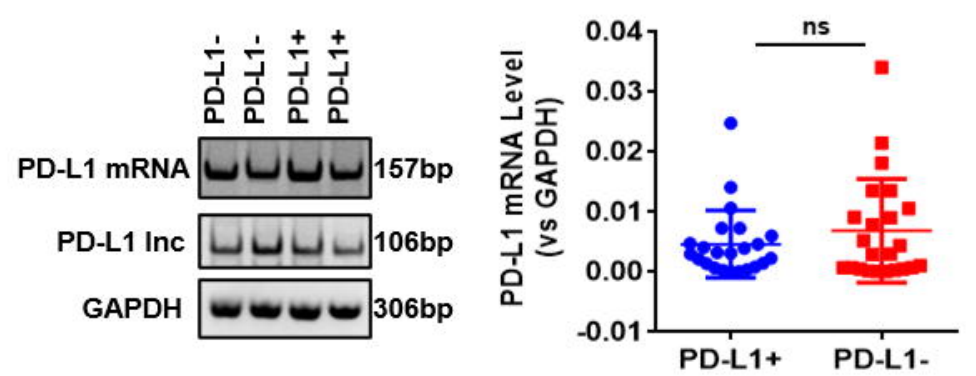

PD-L1-Inc primer 4

$\frac{106 \mathrm{bp}}{\text { Exon3 Exon4 Exon5 Exon6 }}$ 
A

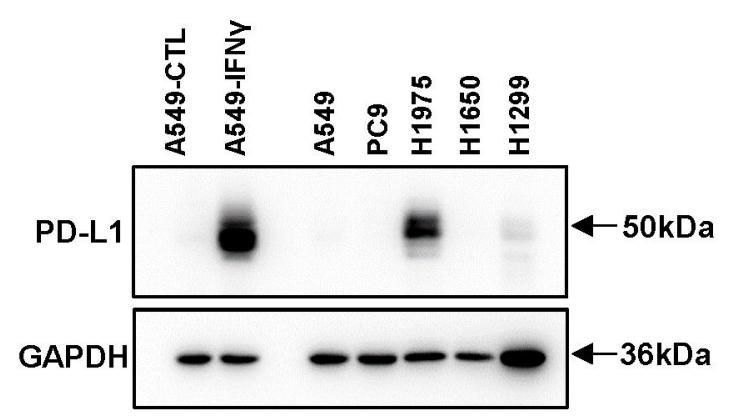

C

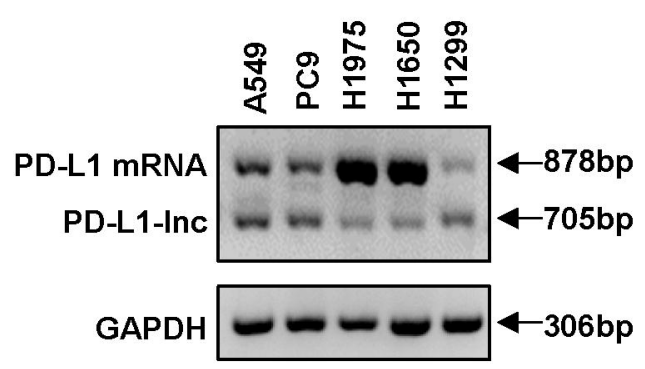

E

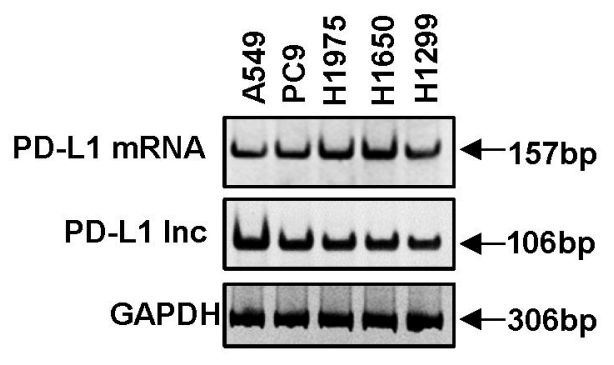

B
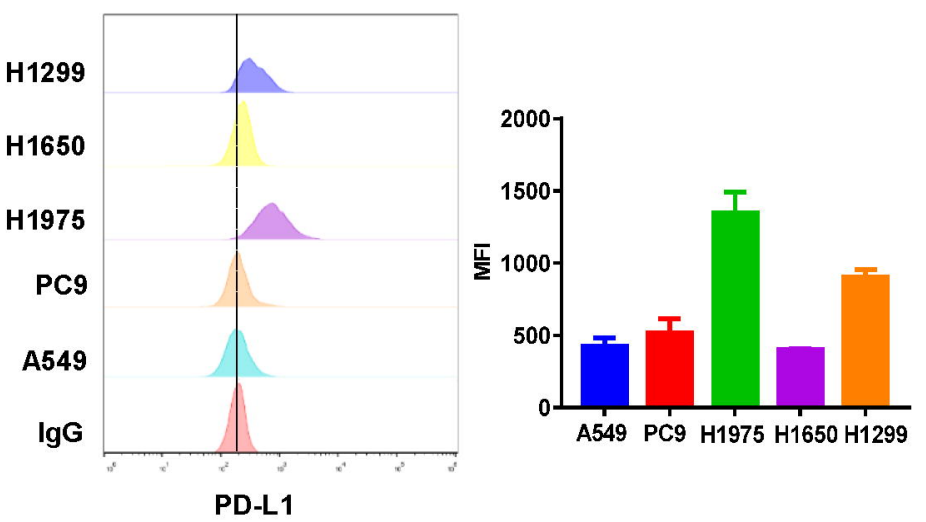

D
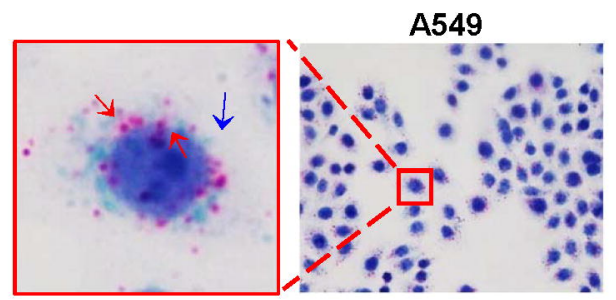

- — PD-L1 mRNA

PC9

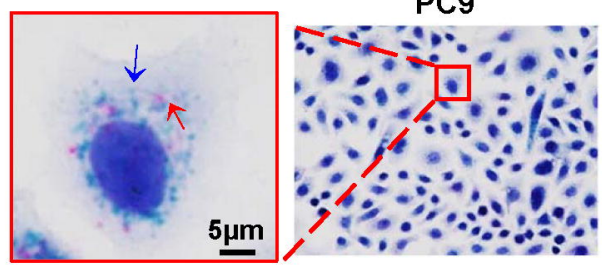

- 4 PD-L1-Inc

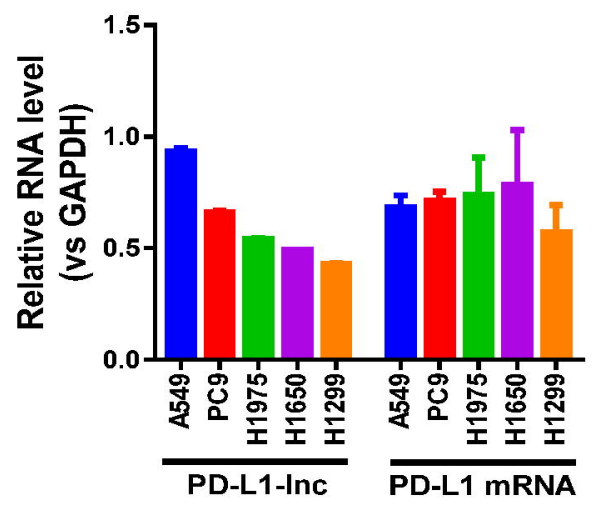


A
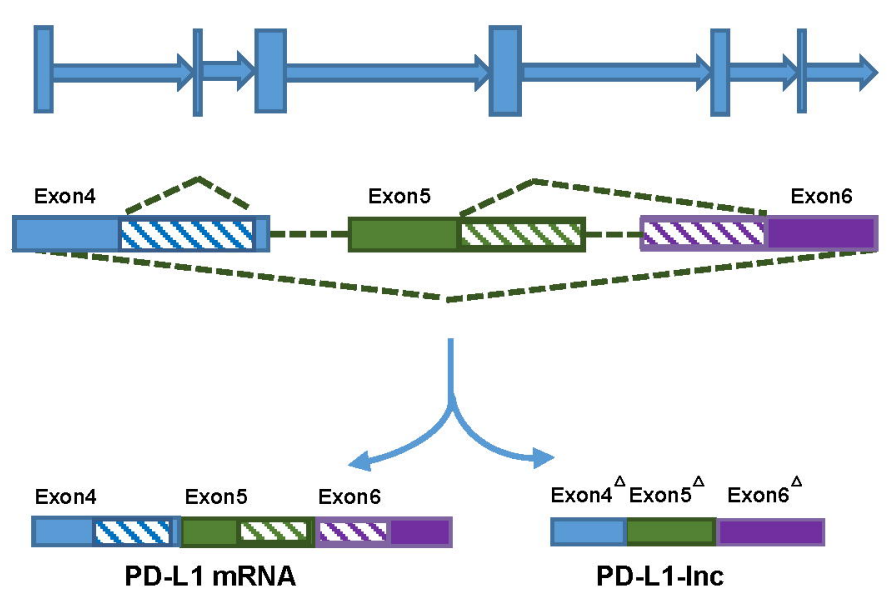

C

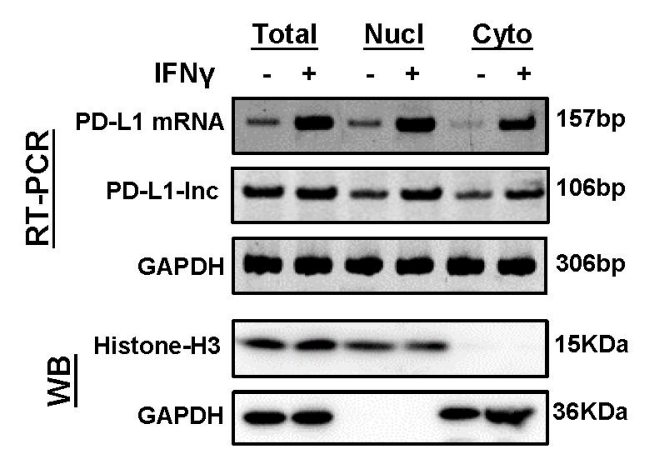

B
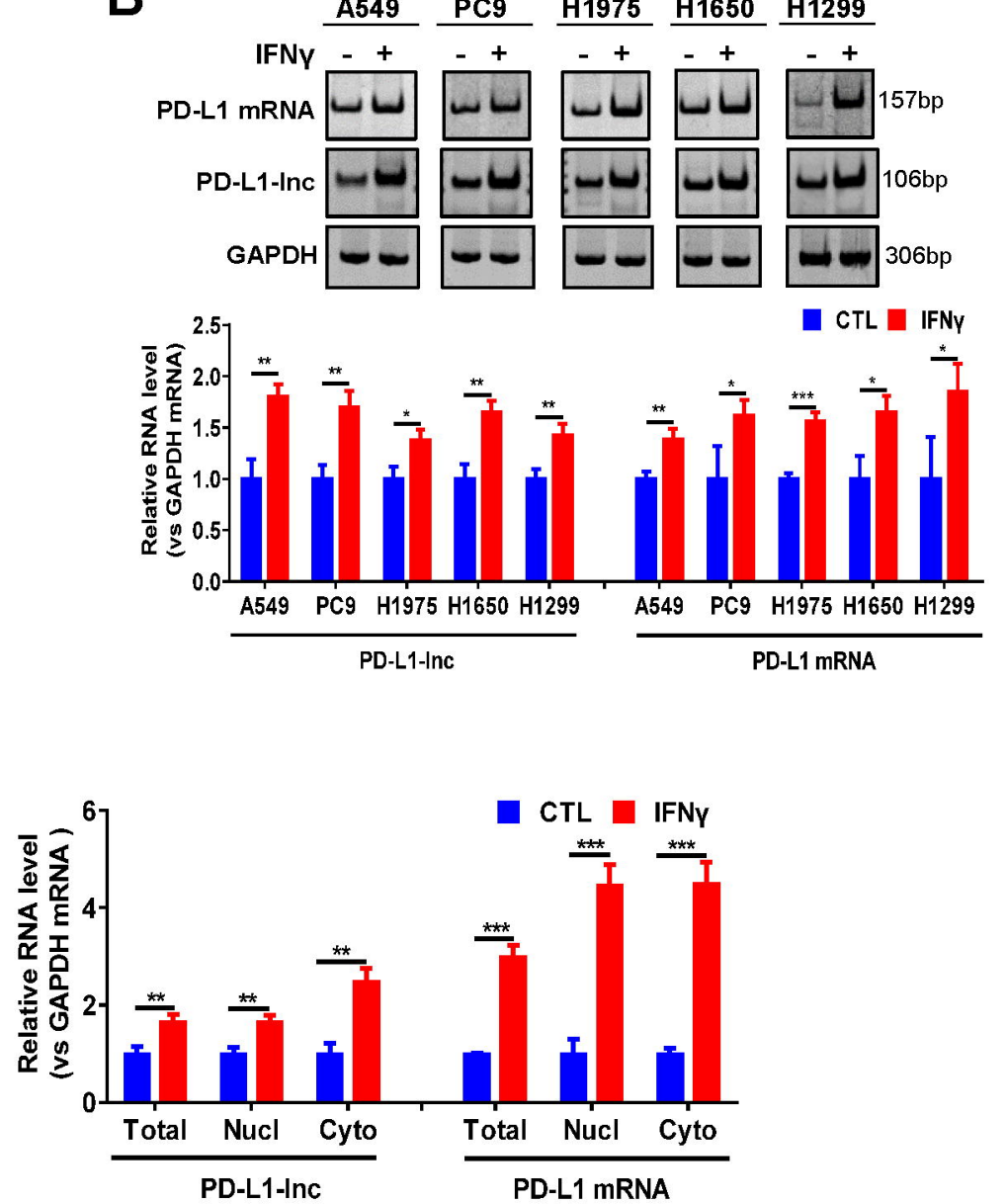
A
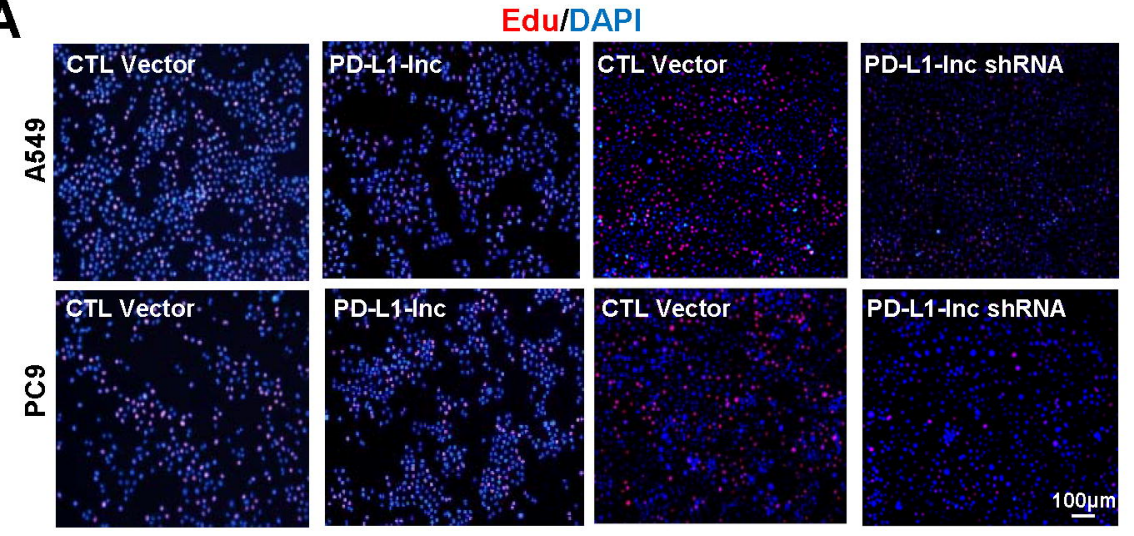

B

CTL Vector

PD-L1-Inc

CTL Vector

PD-L1-Inc ShRNA

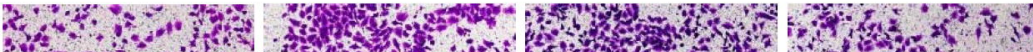

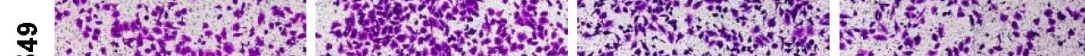
安

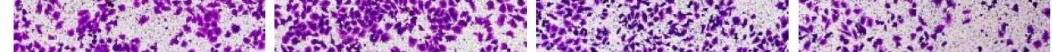

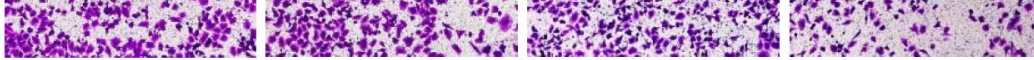
CTL Vector PD-L1-Inc CTL Vector PD-L1-Inc shRNA

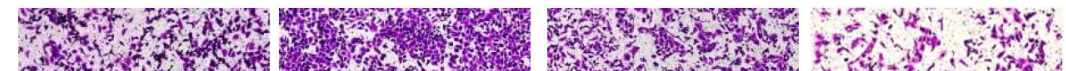

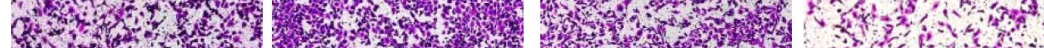

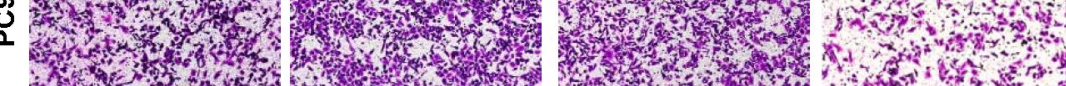

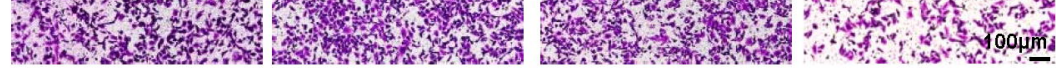

C

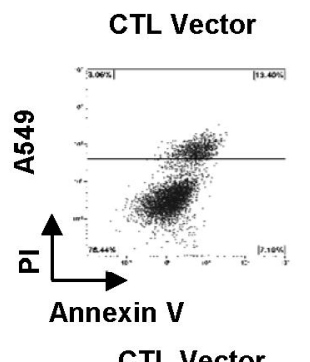

PD-L1-Inc

CTL Vector

PD-L1-Inc shRNA CTL Vector
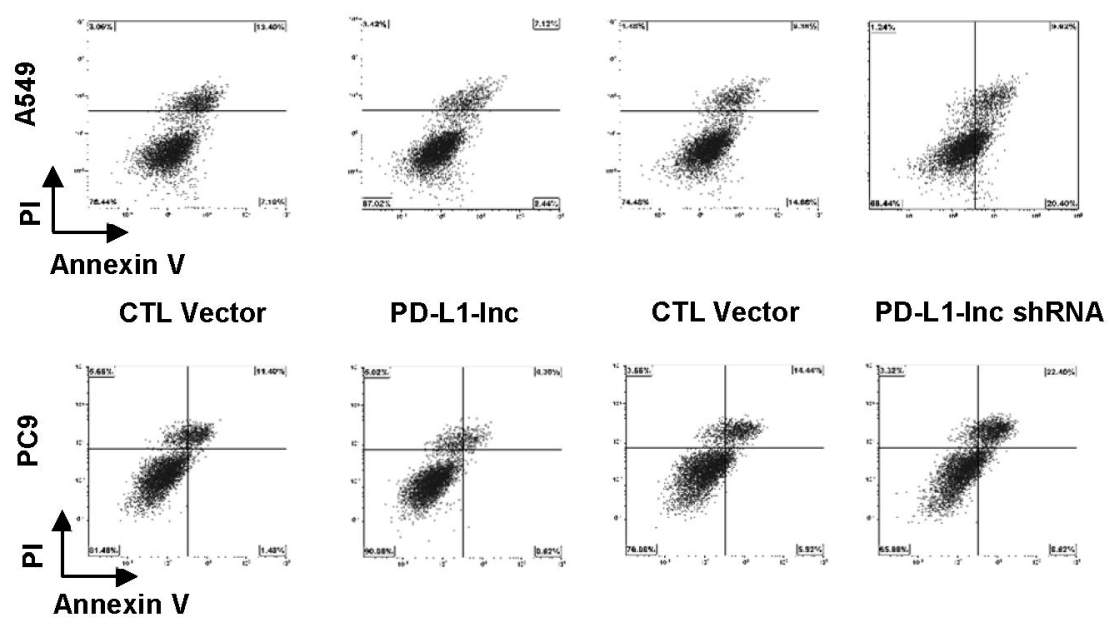

CTL Vector

PD-L1-Inc shRNA

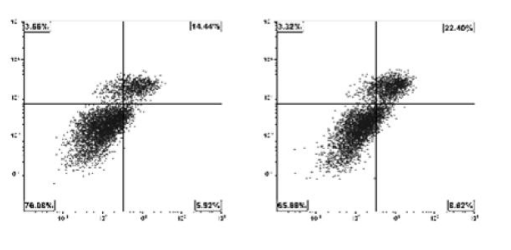

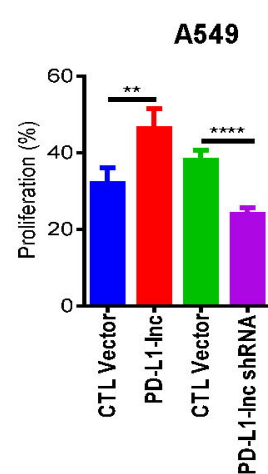
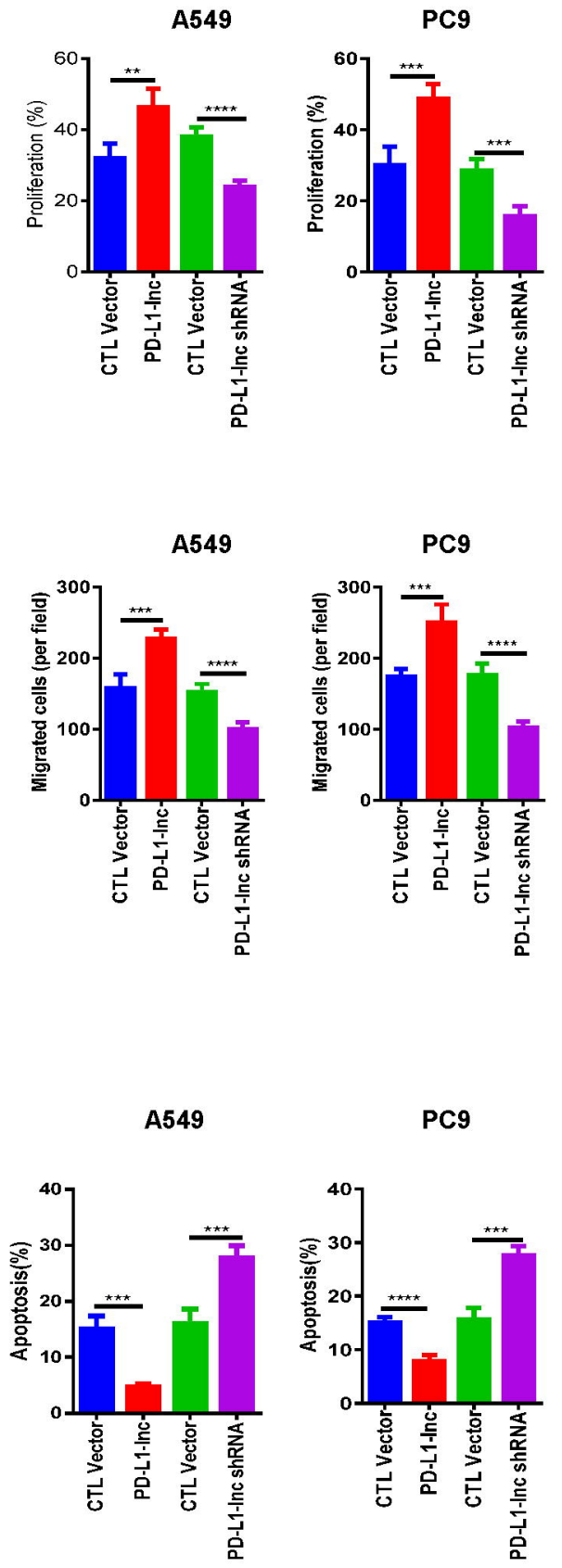
A

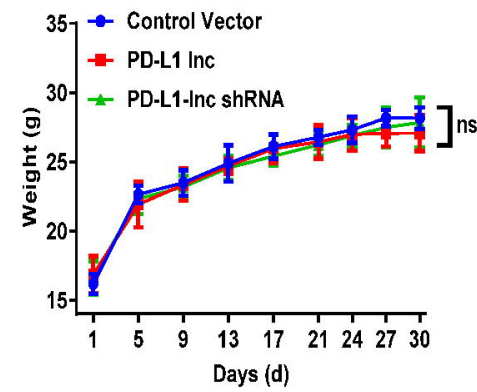

D
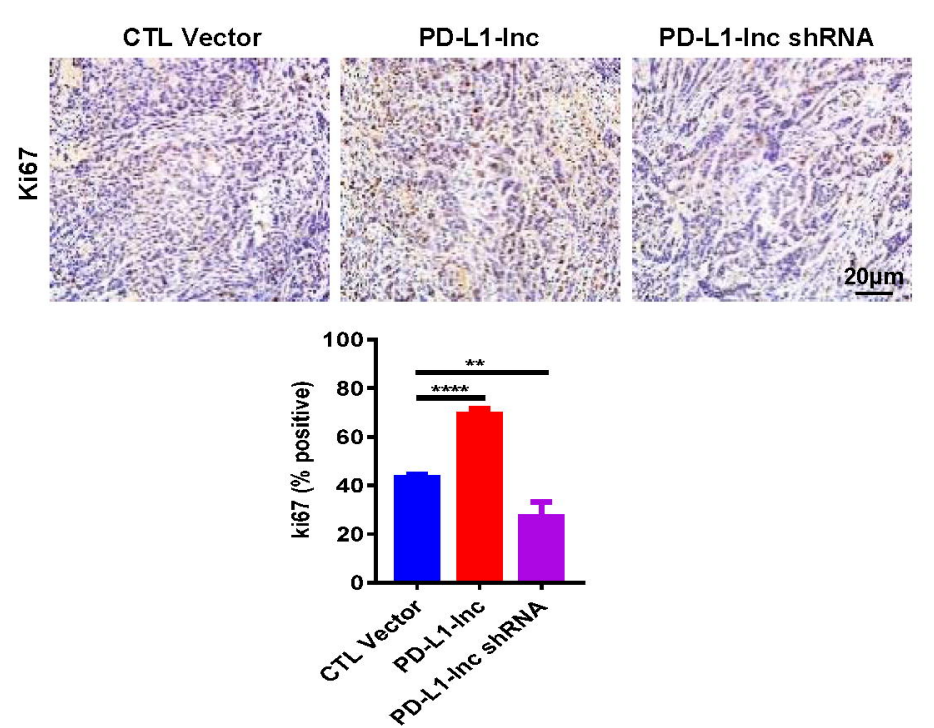

C

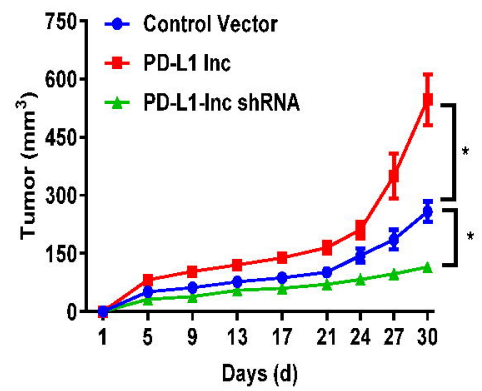

$E$

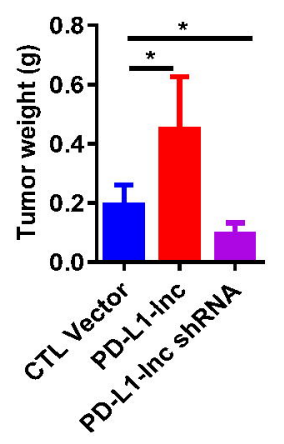

CTL Vector PD-L1-Inc PD-L1-Inc shRNA

PD-L1-Inc

PD-L1-mRNA $---\infty---\infty-\infty----$

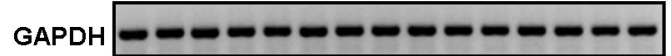

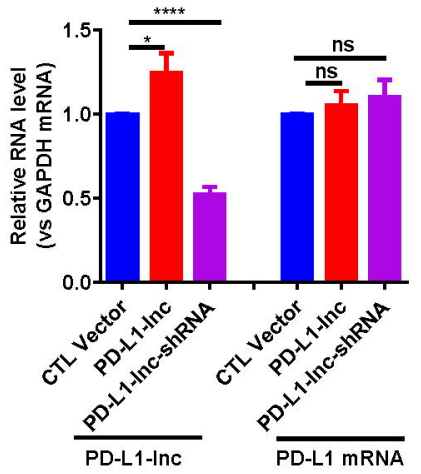


A

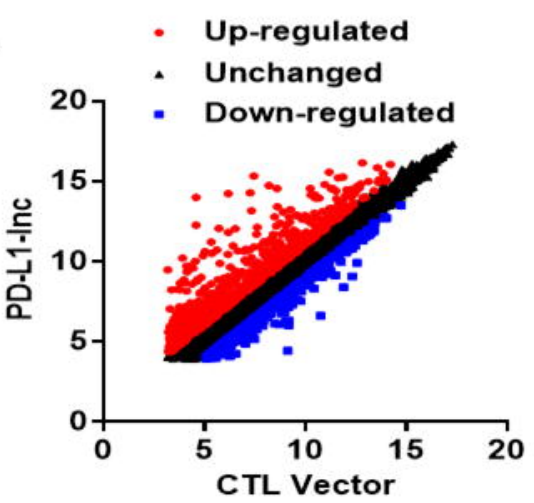

B

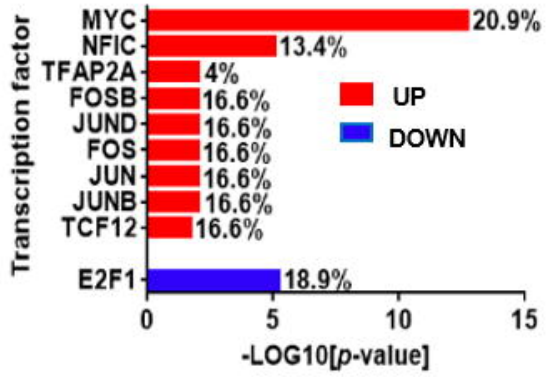

C

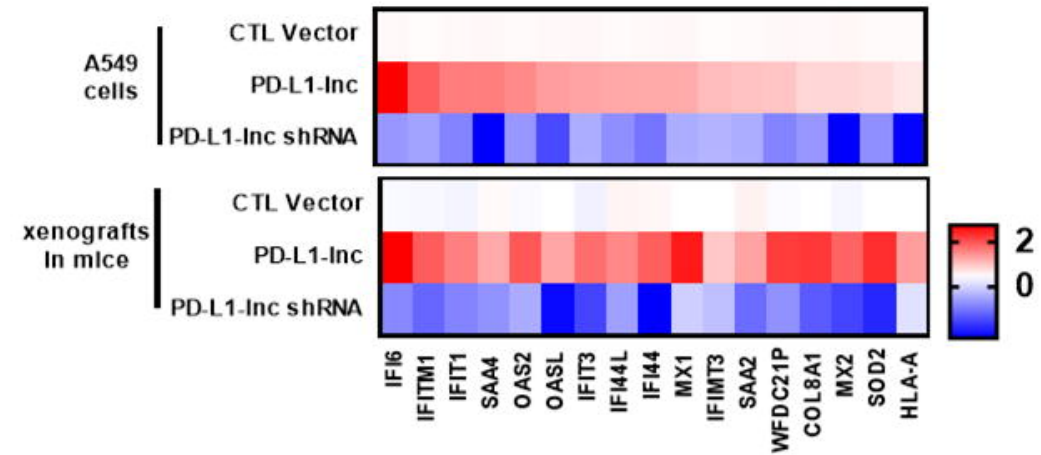

D

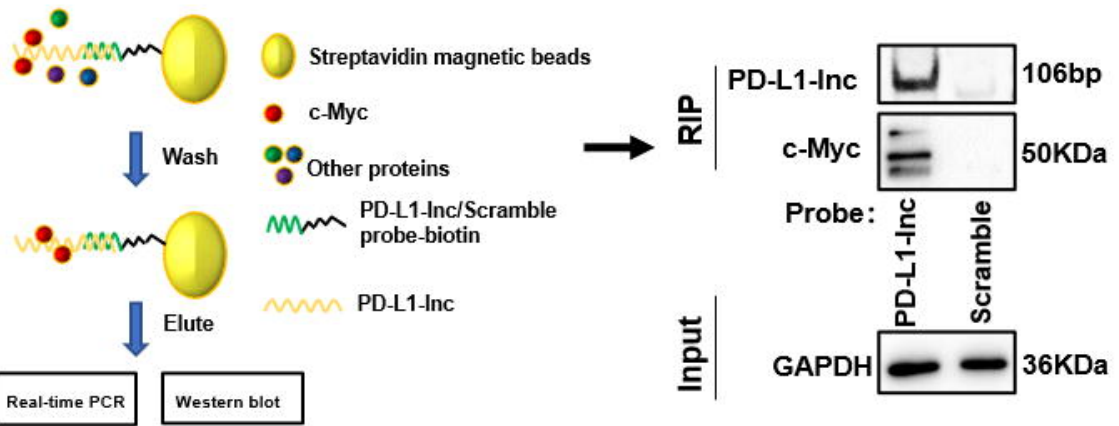

E

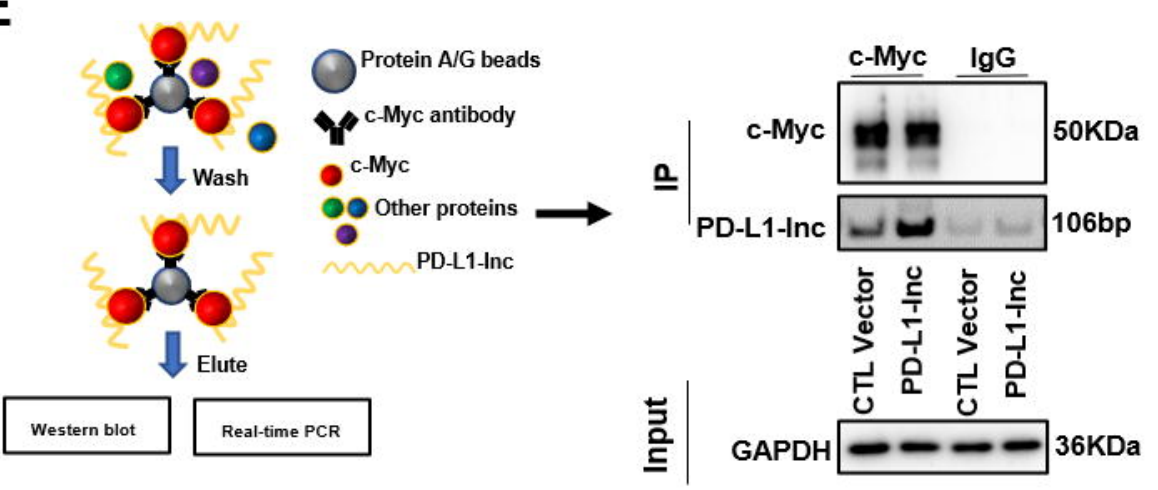




\section{A}

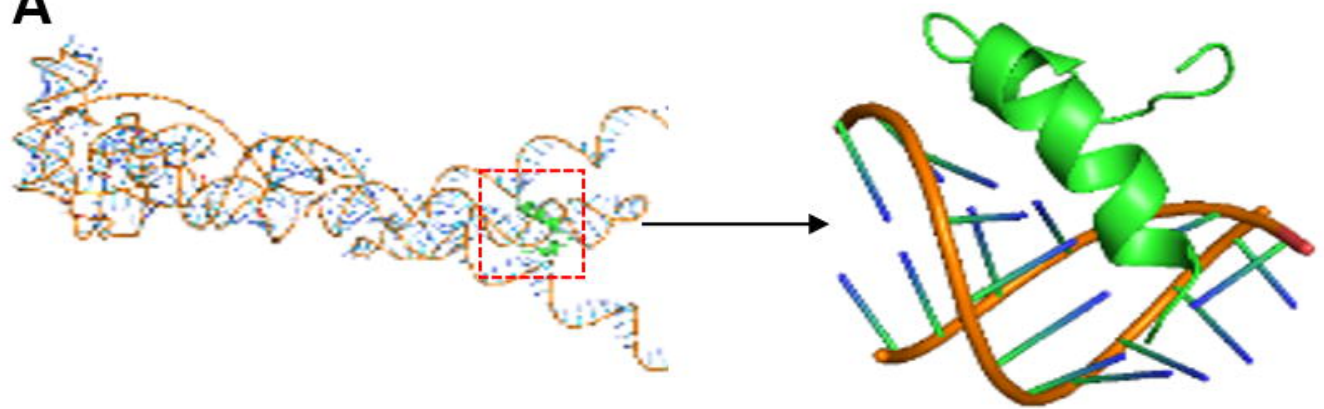

THR(117st)-AU(880-881st)
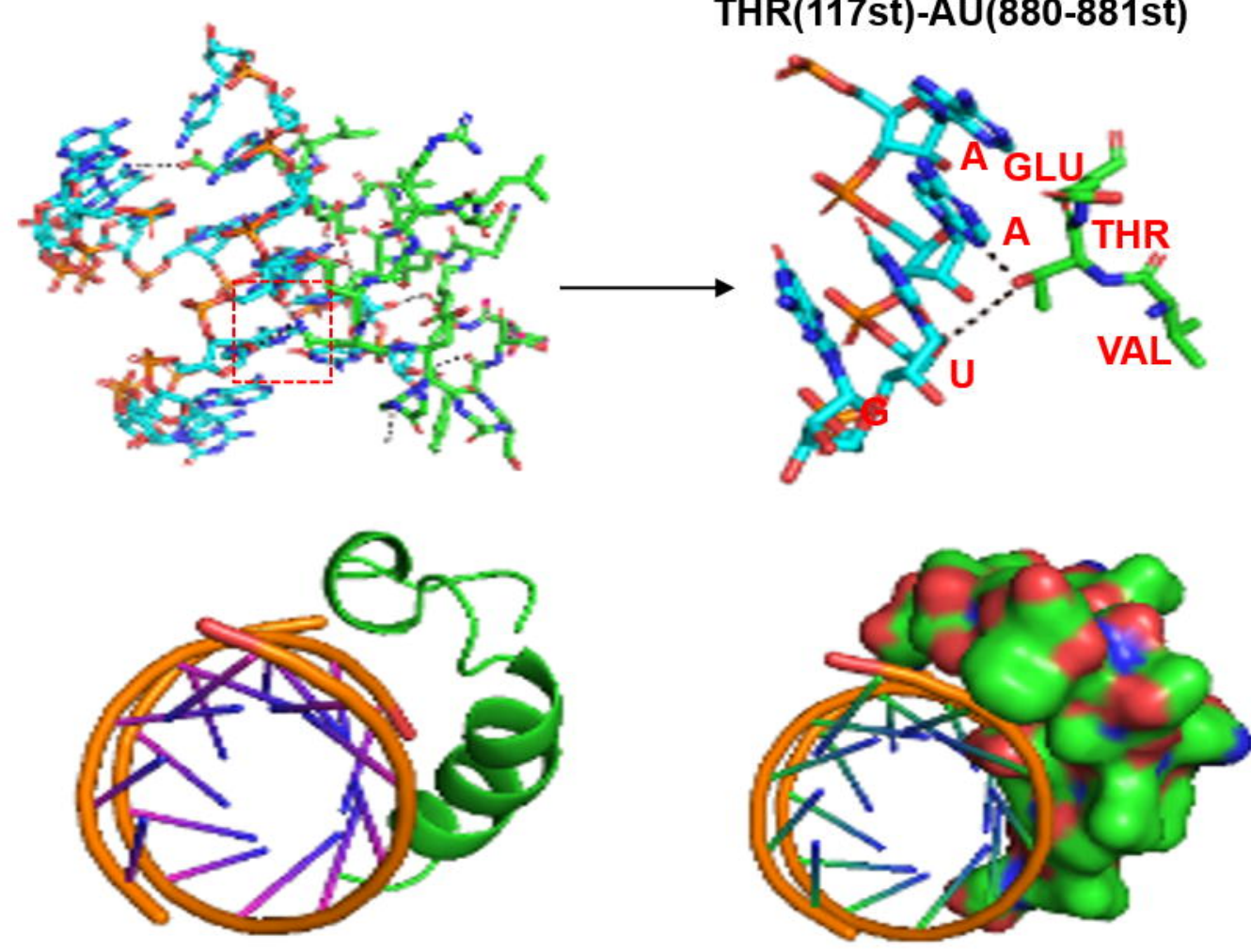

C

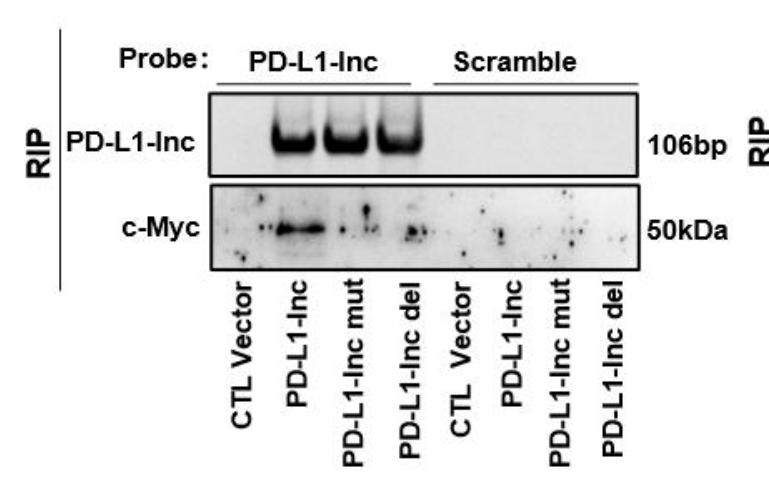

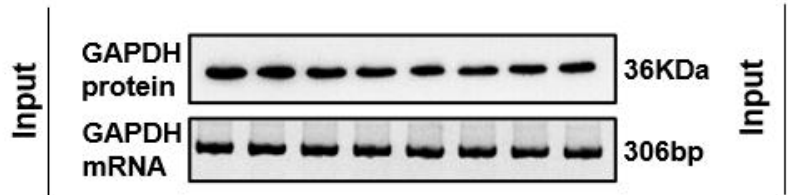

D
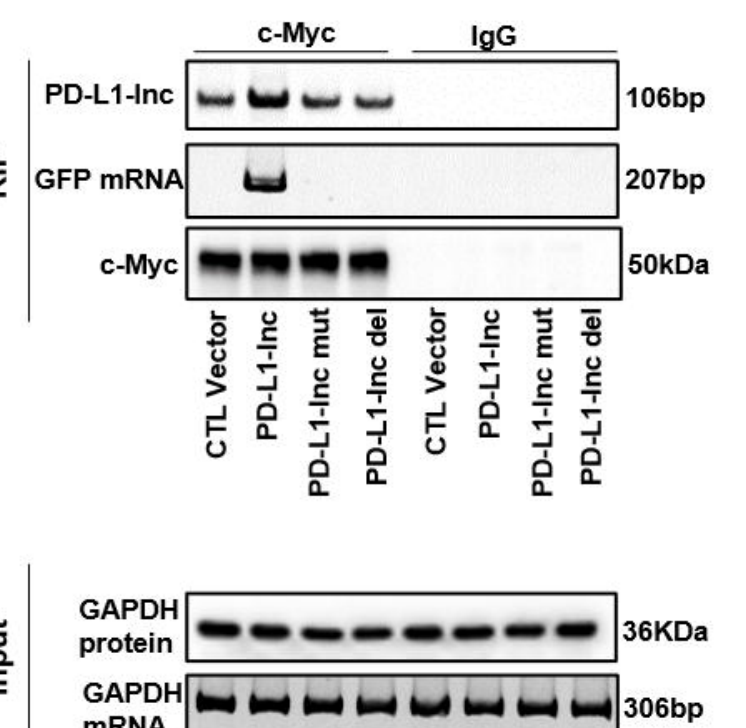

PD-L1-Inc GATGCAGGCAATGTGGGACTTAAAAGGCCCAAGCACTGAAA PD-L1-Inc mut GATGCAGCCATACTGGGACTTAAAAGGCCCATCCACTGAAA PD-L1-Inc del GATGCA------ACTGAAA
E

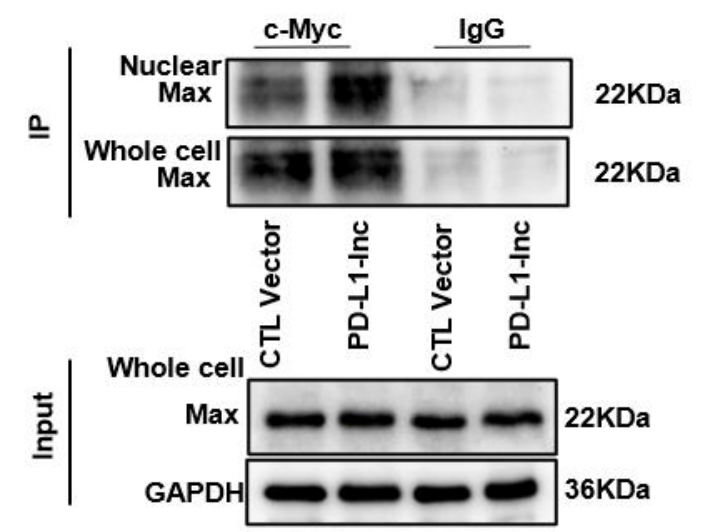

$\mathbf{F}$

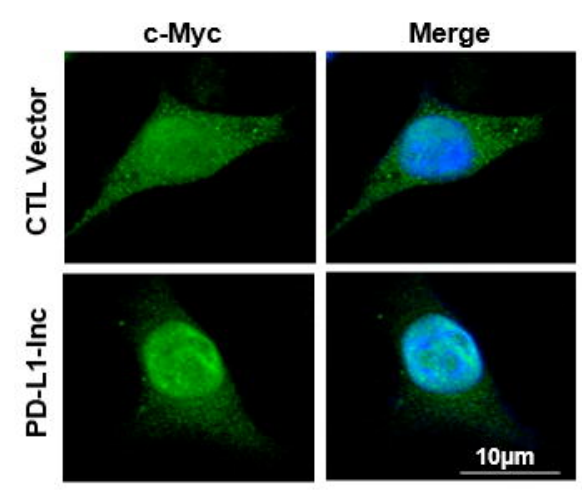

$\mathbf{G}$

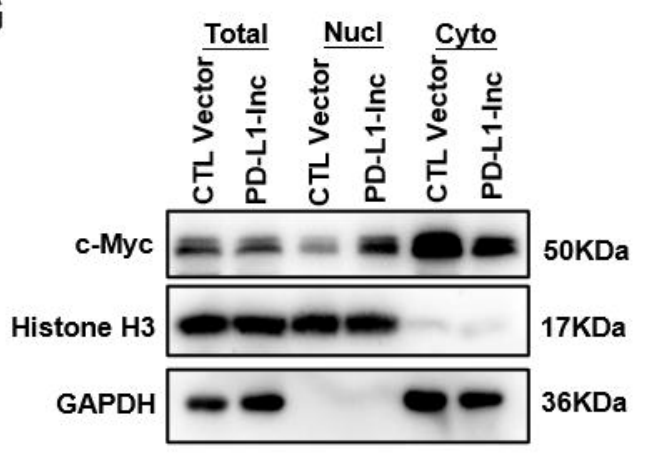

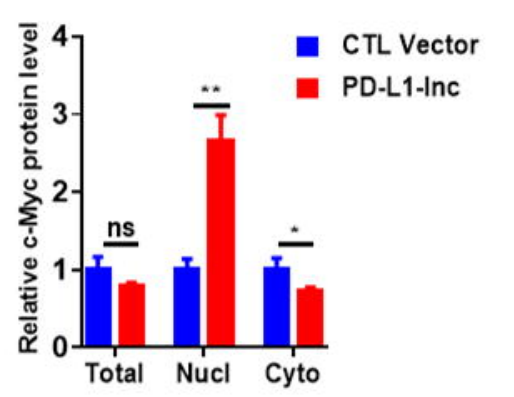


A
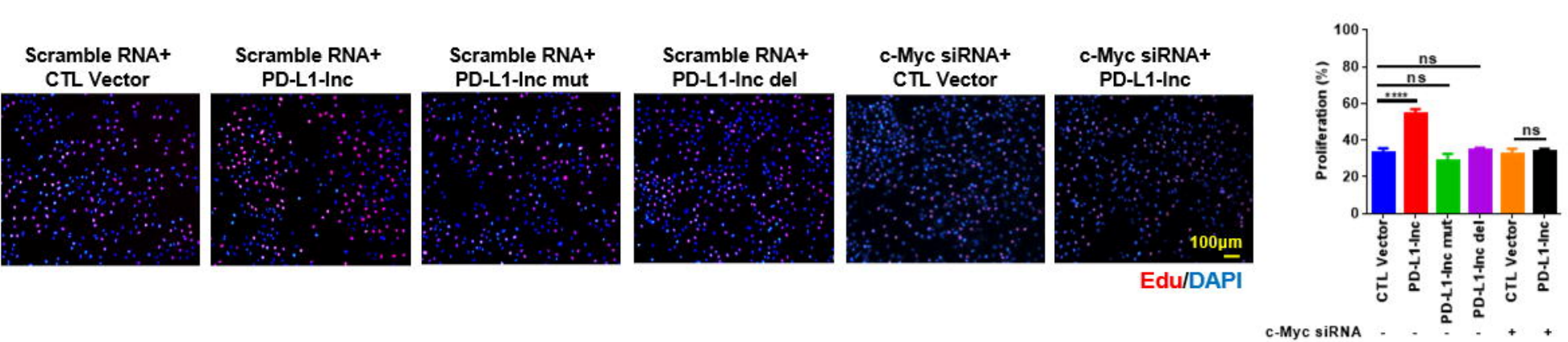

B

$\begin{array}{cccccc}\begin{array}{c}\text { Scramble RNA+ } \\ \text { CTL Vector }\end{array} & \begin{array}{c}\text { Scramble RNA+ } \\ \text { PD-L1-Inc }\end{array} & \begin{array}{c}\text { Scramble RNA+ } \\ \text { PD-L1-Inc mut }\end{array} & \begin{array}{c}\text { Scramble RNA+ } \\ \text { PD-L1-Inc del }\end{array} & \begin{array}{c}\text { c-Myc siRNA+ } \\ \text { CTL Vector }\end{array} & \begin{array}{c}\text { c-Myc siRNA+ } \\ \text { PD-L1-Inc }\end{array}\end{array}$

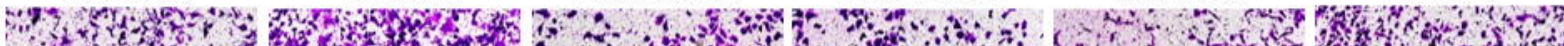

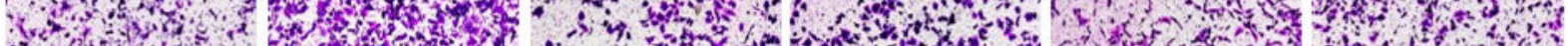

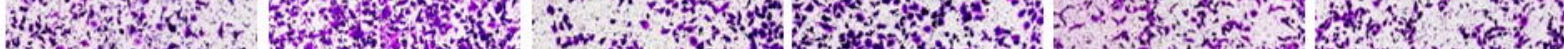

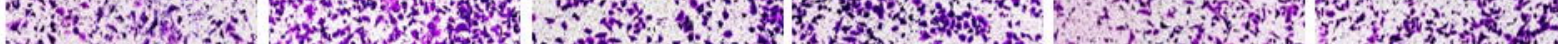

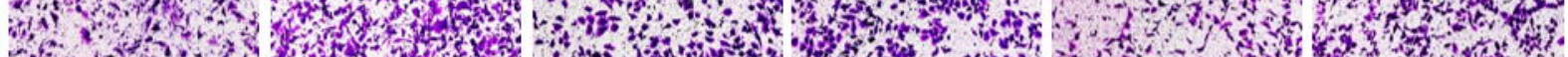

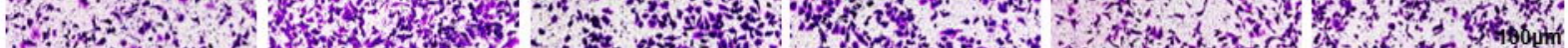

c-Myc SiRNA

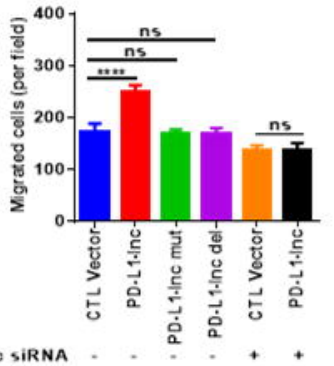

C

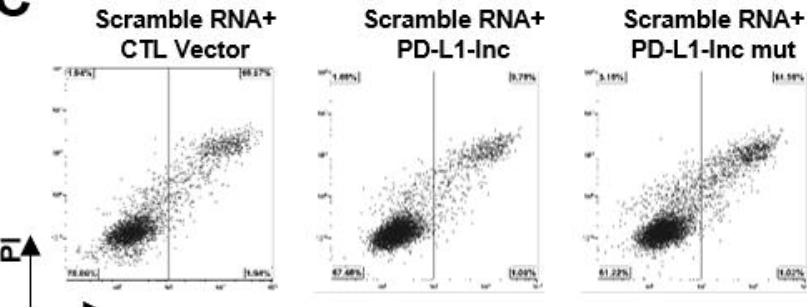
Annexin V
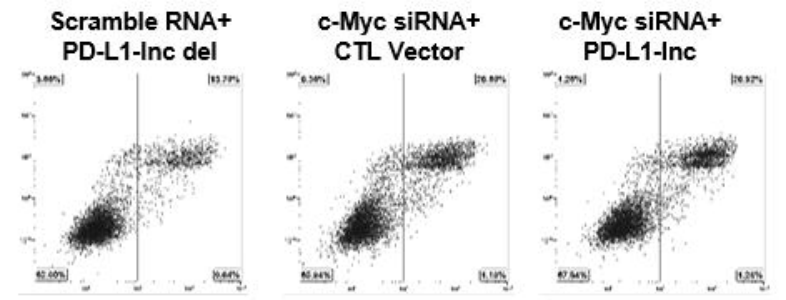

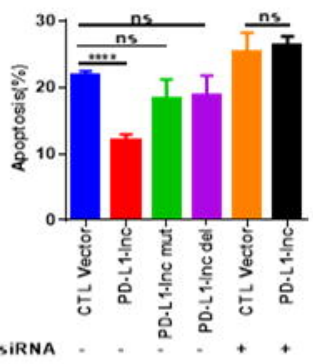




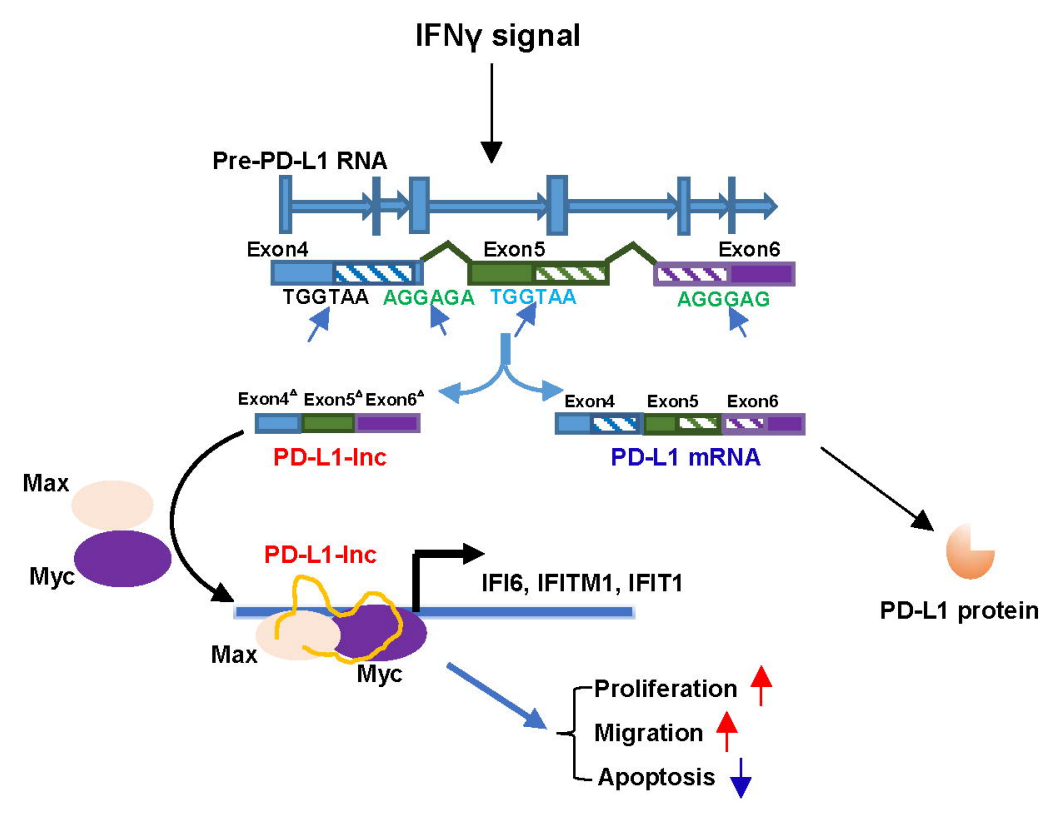


A

PD-L1+

PD-L1-

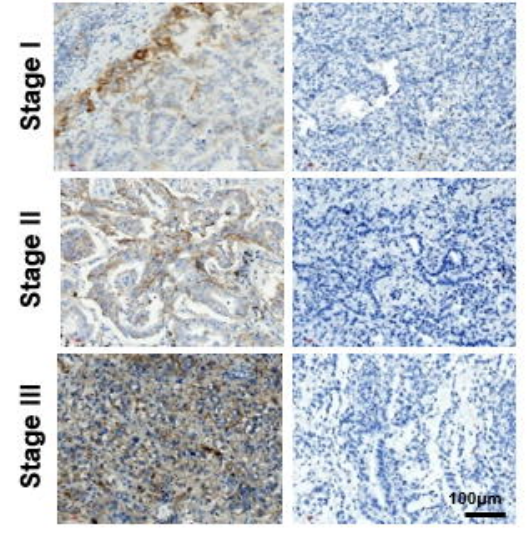

B

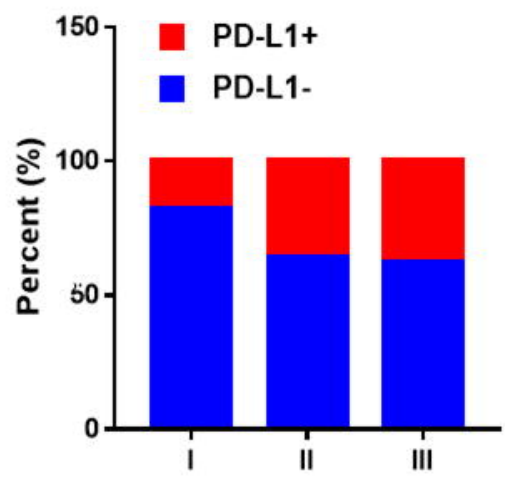

PD-L1 primer 1

PD-L1 mRNA

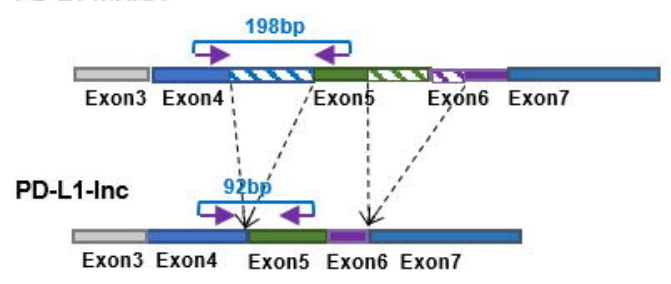


A

PD-L1 mRNA

\section{Amplicon Size $\quad 878 \mathrm{bp}$}

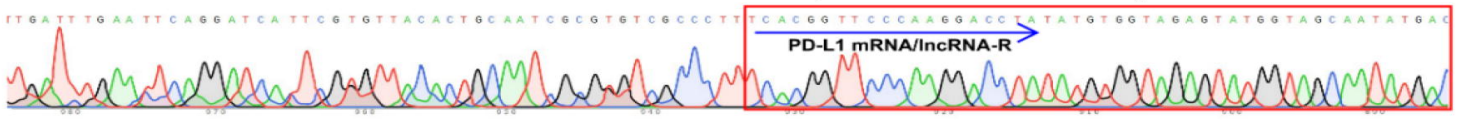

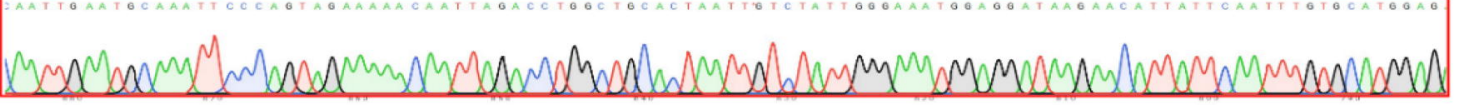

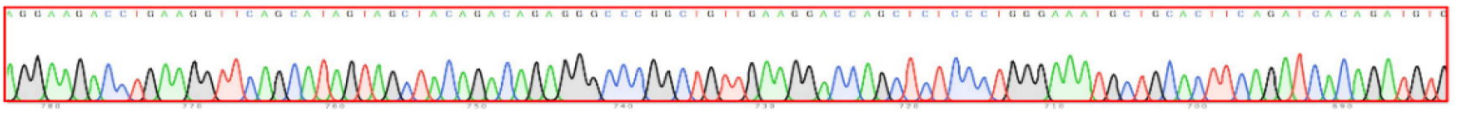

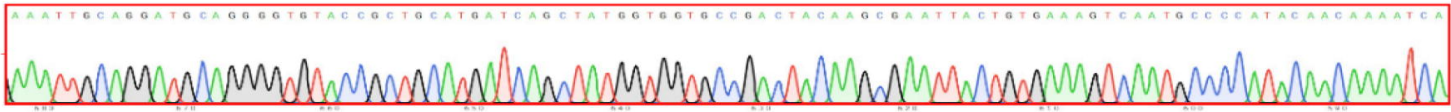
Wh

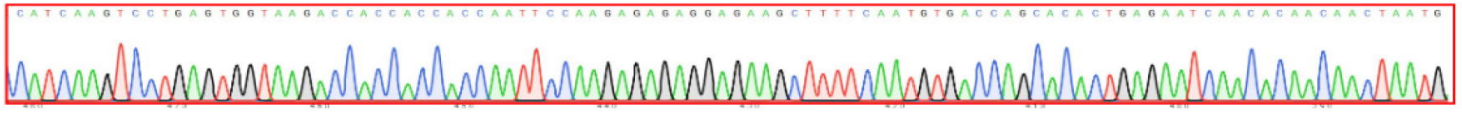

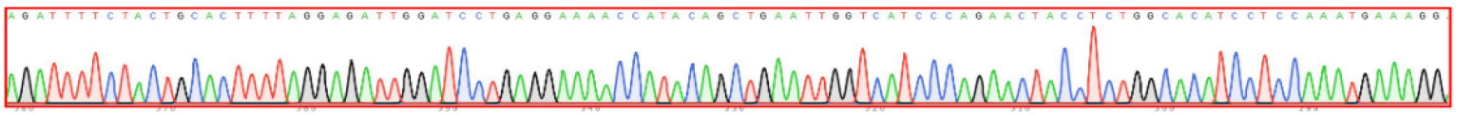

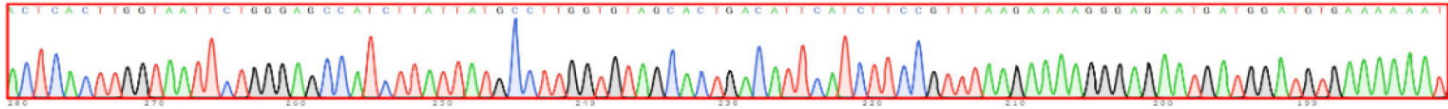
CoWhWh

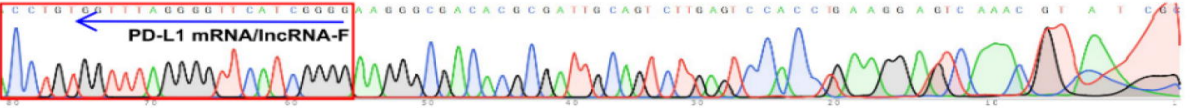

B PD-L1-Inc

Amplicon Size $705 \mathrm{bp}$

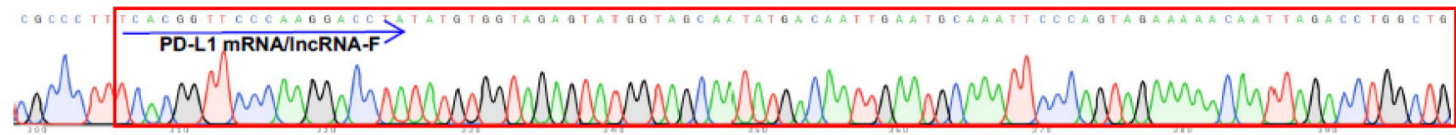

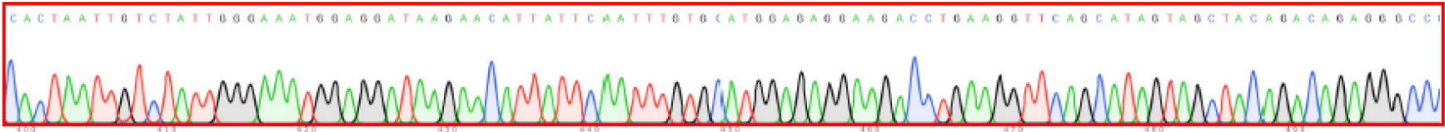

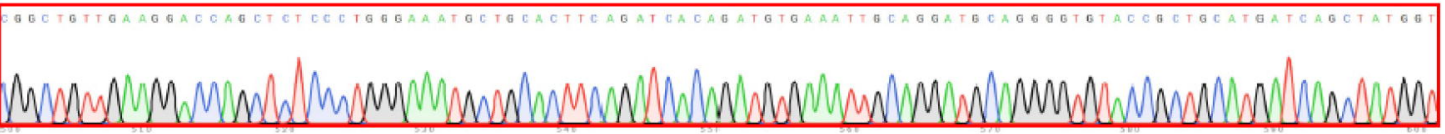

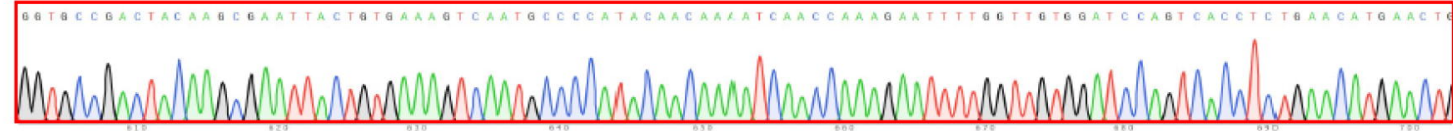

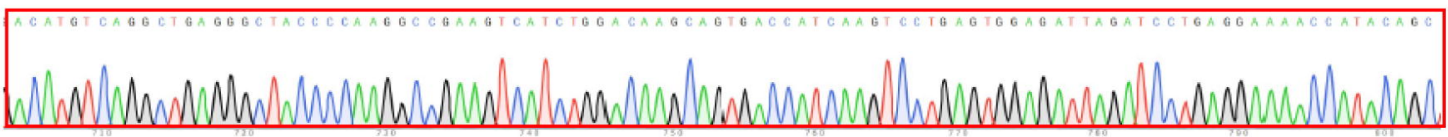

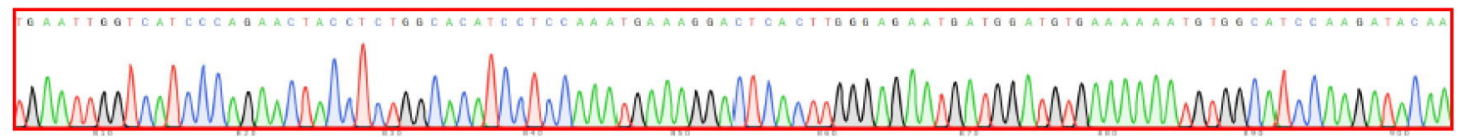

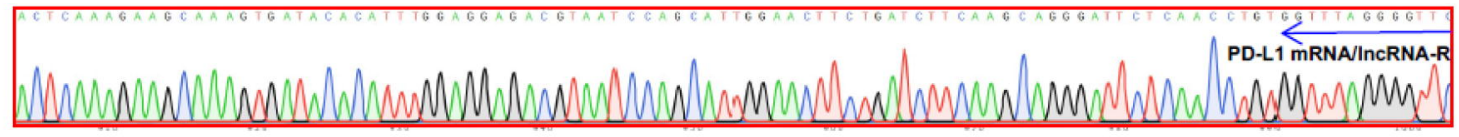

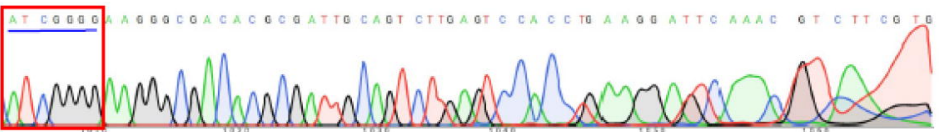


A

PD-L1 mRNA

Amplicon Size $157 \mathrm{bp}$

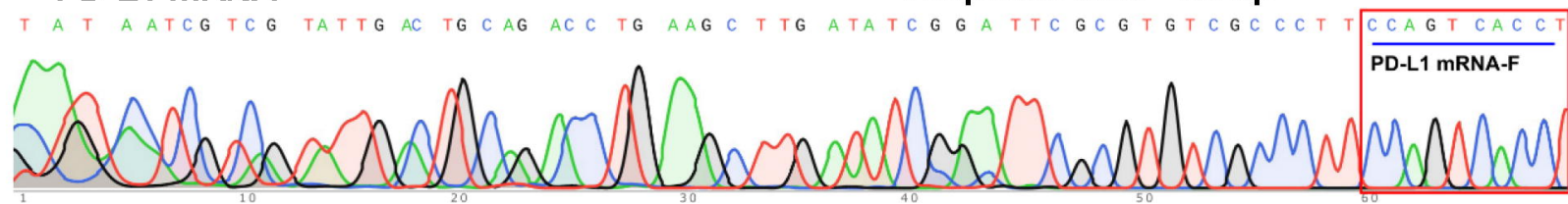

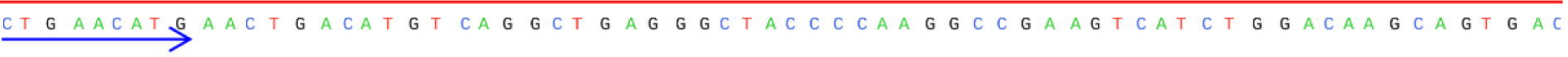

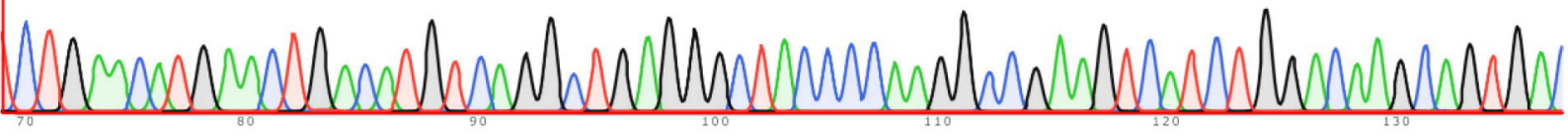

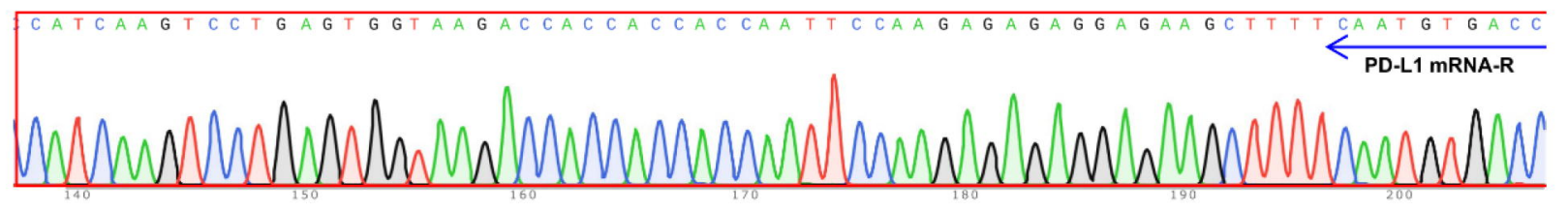

B

PD-L1-Inc

Amplicon Size $106 \mathrm{bp}$
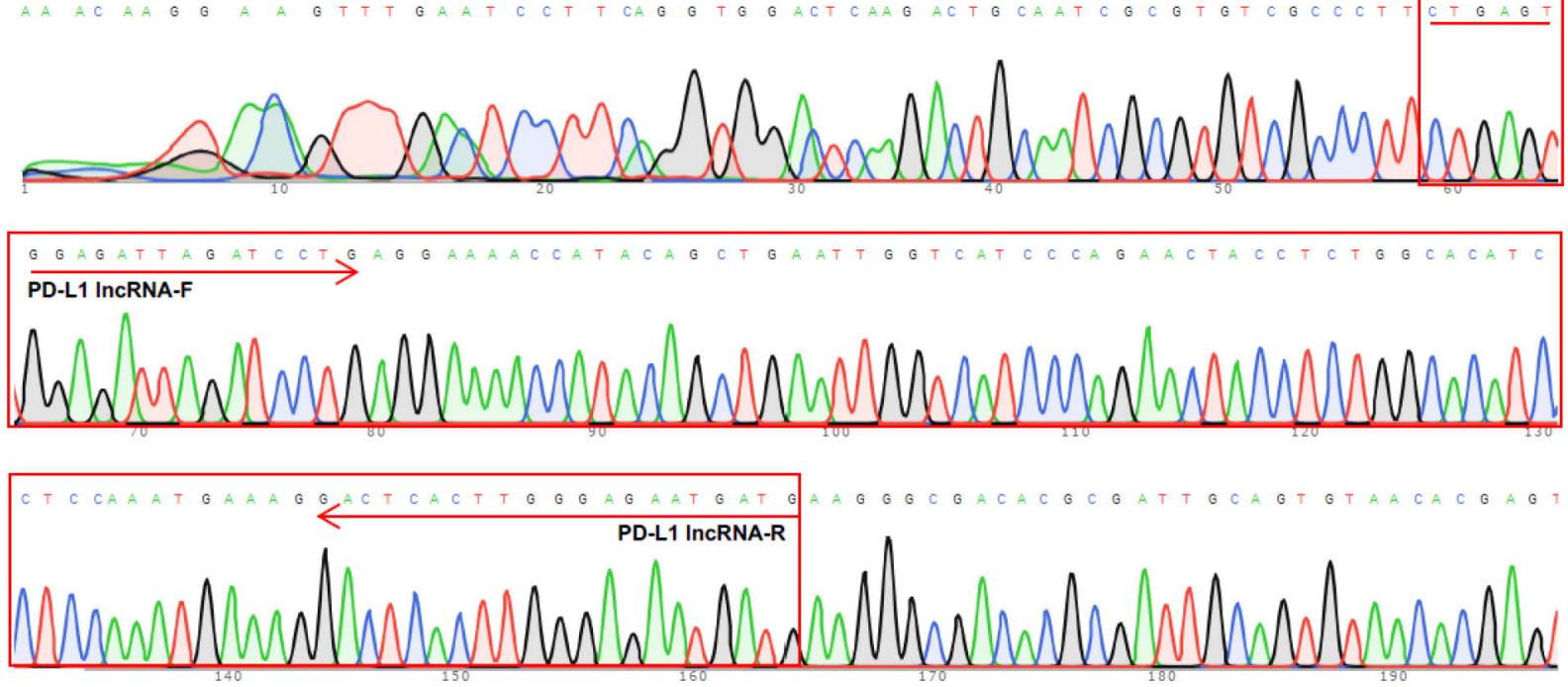
A

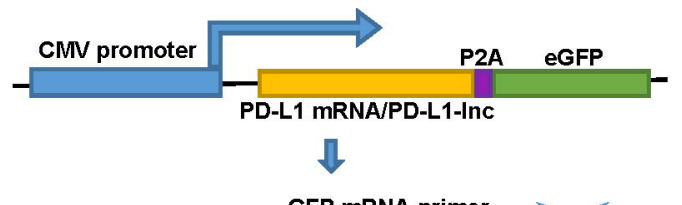

CTL Vector GFP mRNA primer) PD-L1 mRNA PD-L1-Inc Nm

$$
\begin{aligned}
& \text { 害 } \\
& \text { 品 }
\end{aligned}
$$

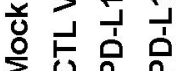

$$
\begin{aligned}
& \text { GFP mRNA } \\
& \text { घण0 }
\end{aligned}
$$

B

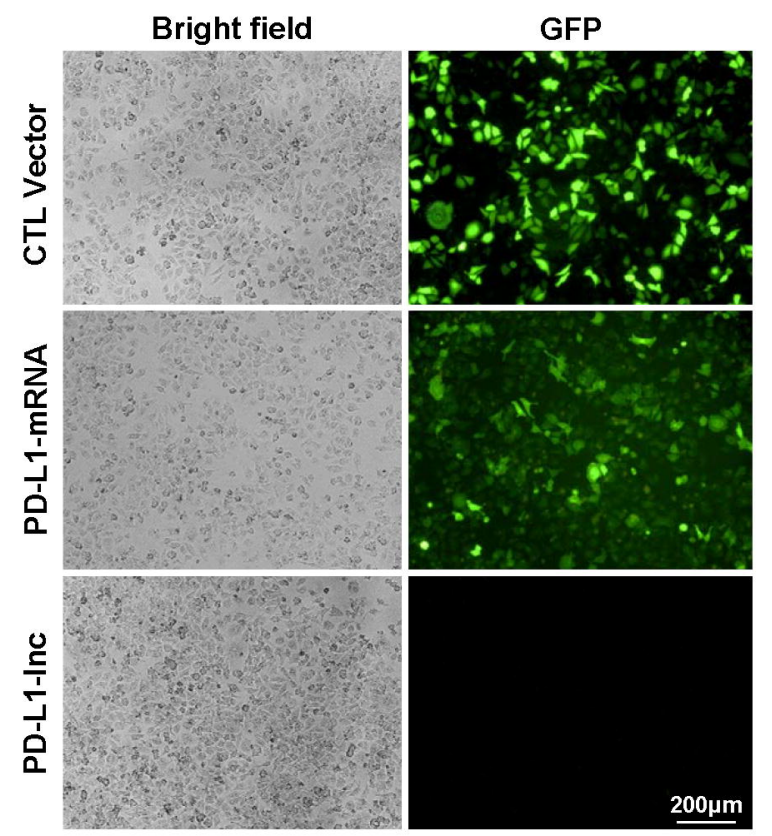


A
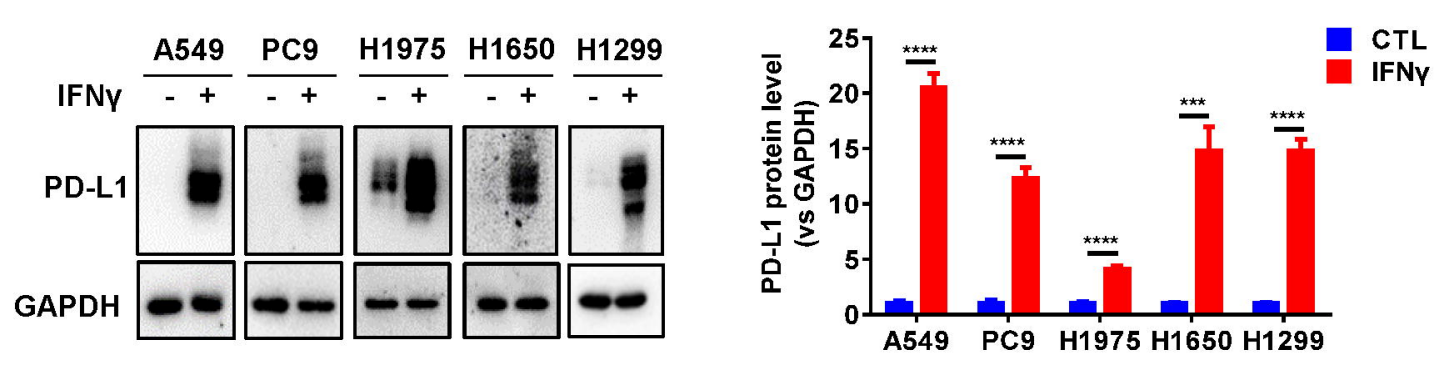

B
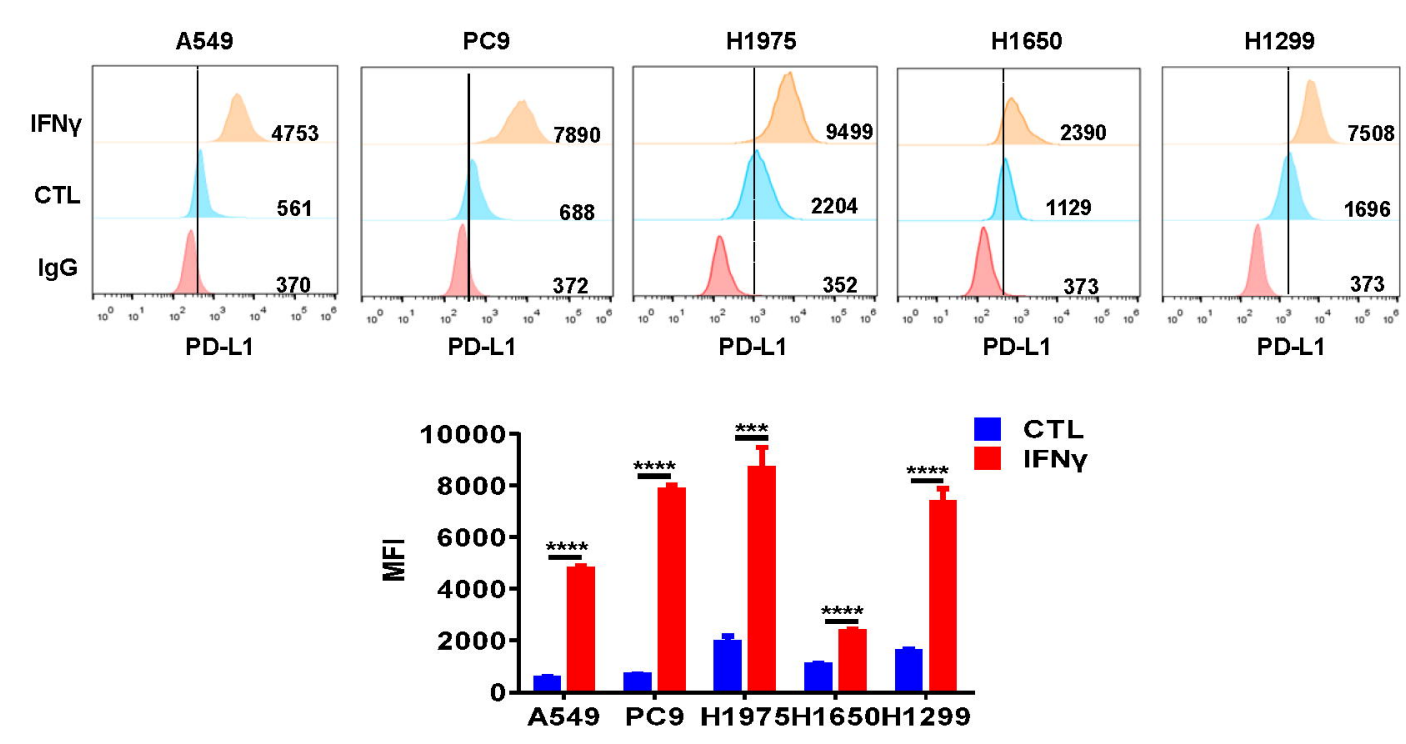
A

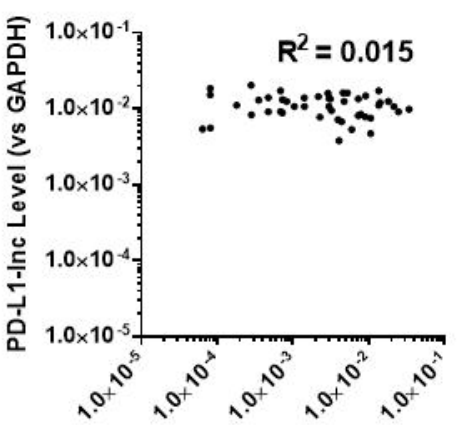

E

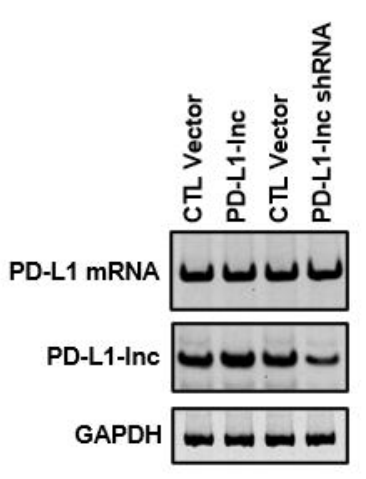

B

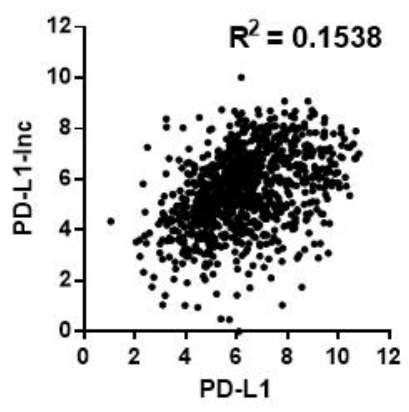

C

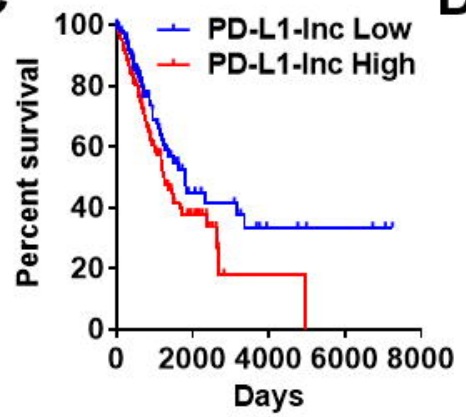

D

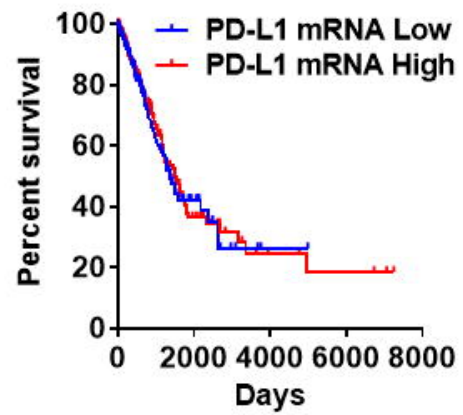

PD-L1 mRNA Level (vs GAPDH)

F

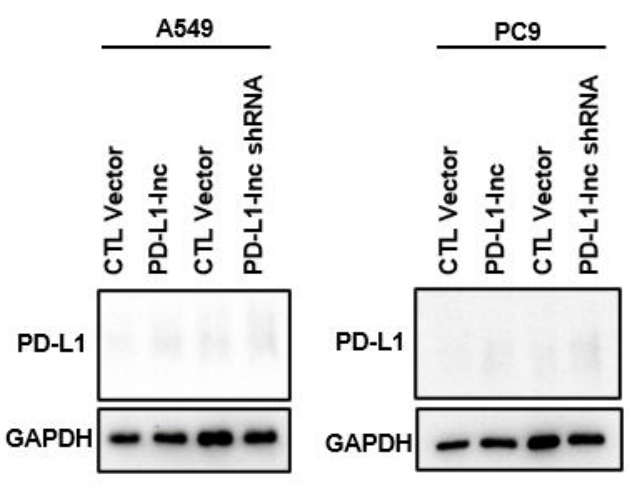

H
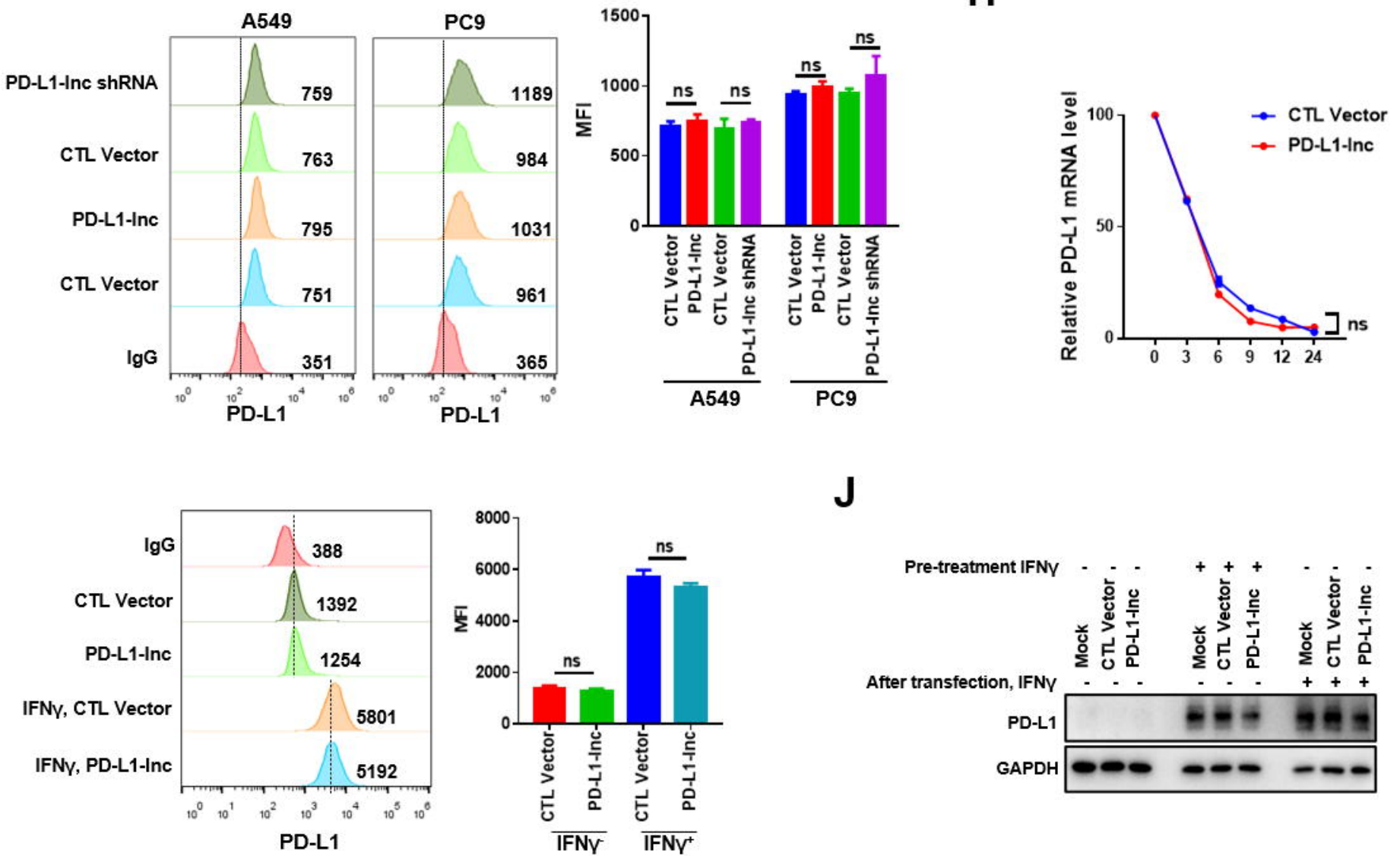

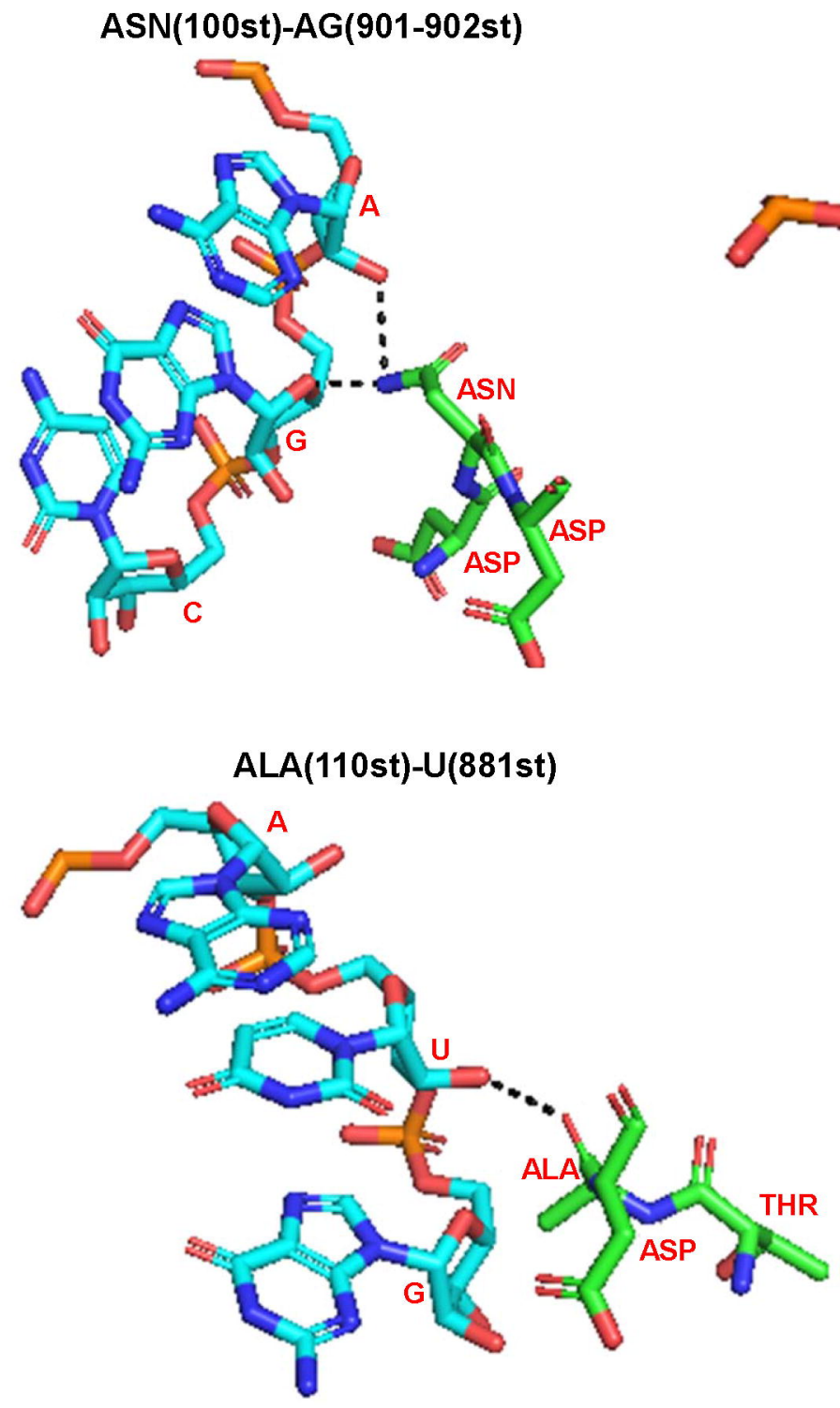

GLY(103st)-U(883st)
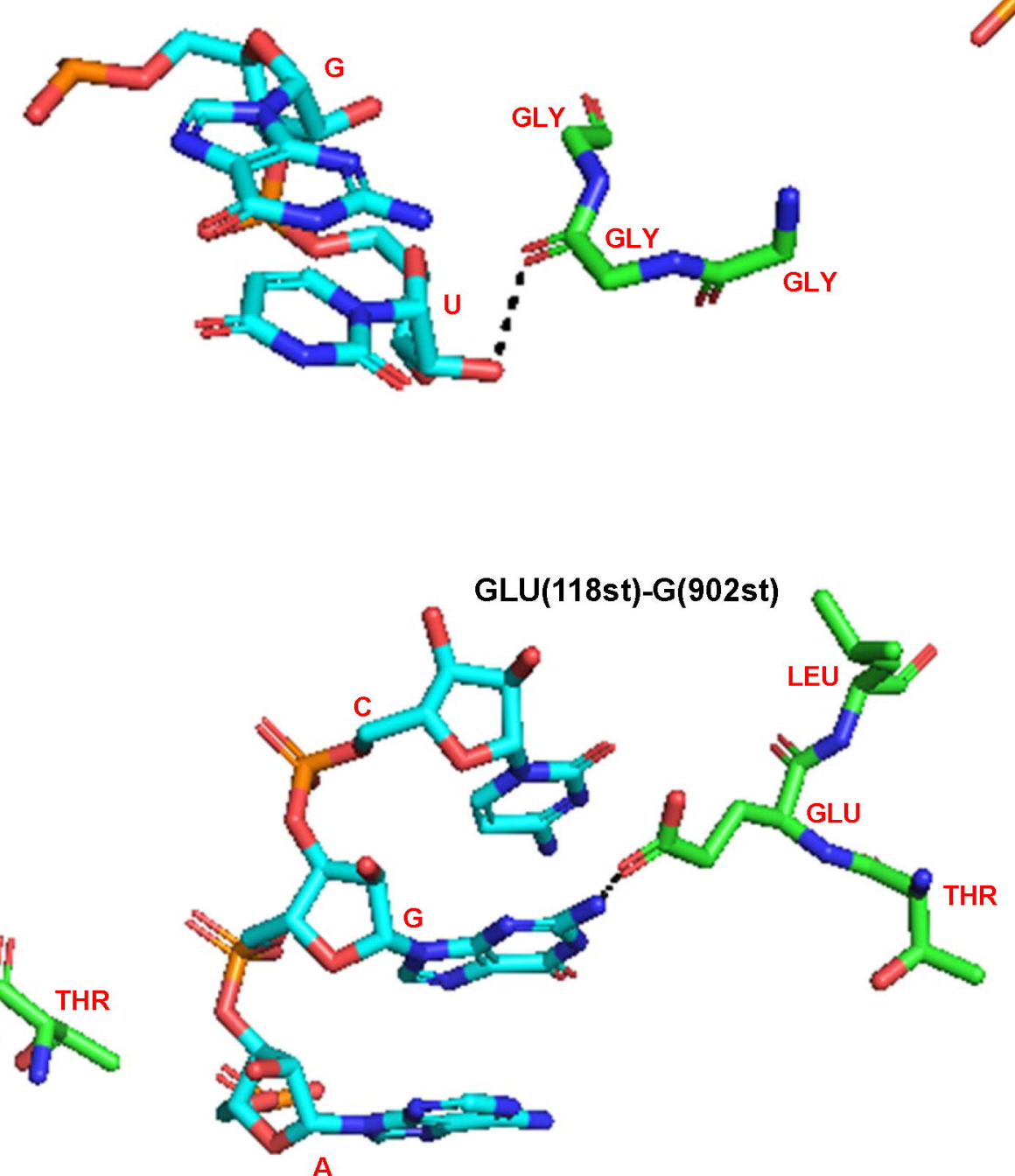

PHE(107st)-G(882st)

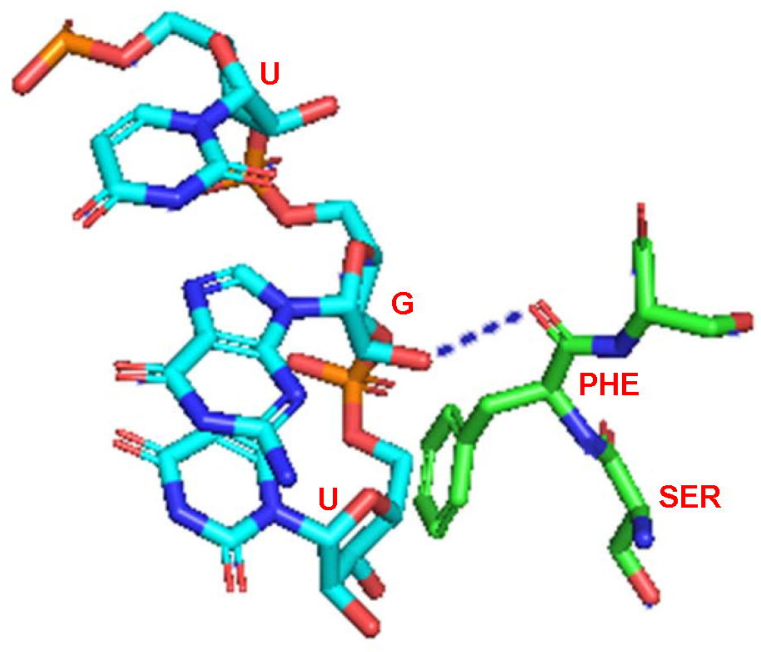

GLY(121st)-G(877st)

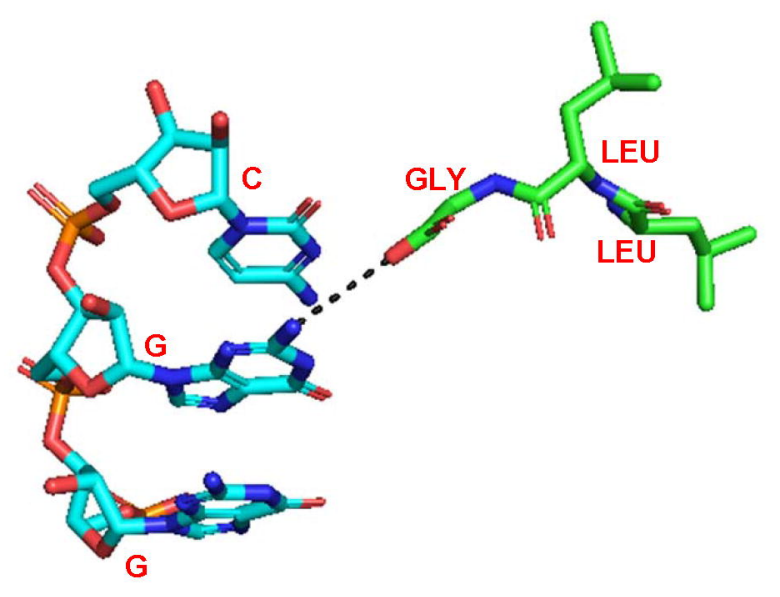



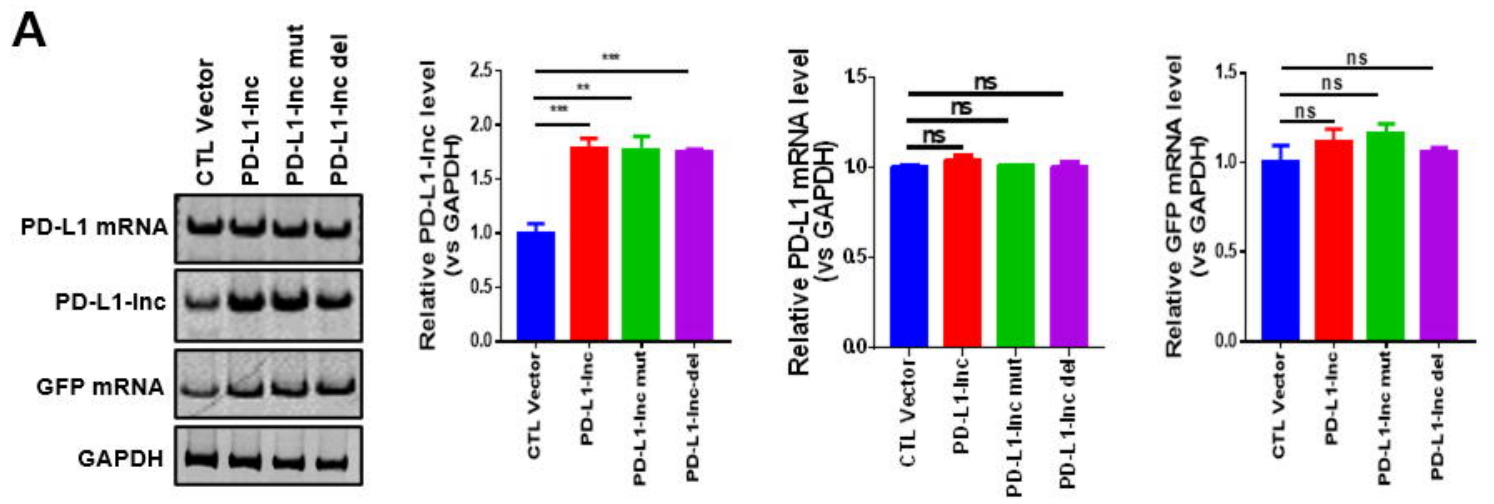

B
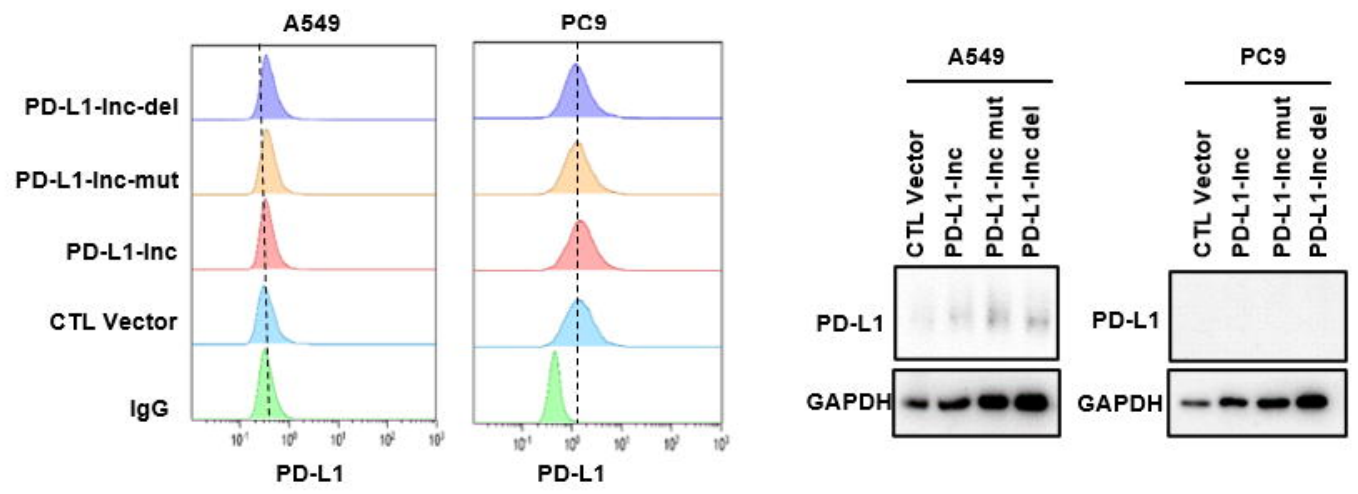

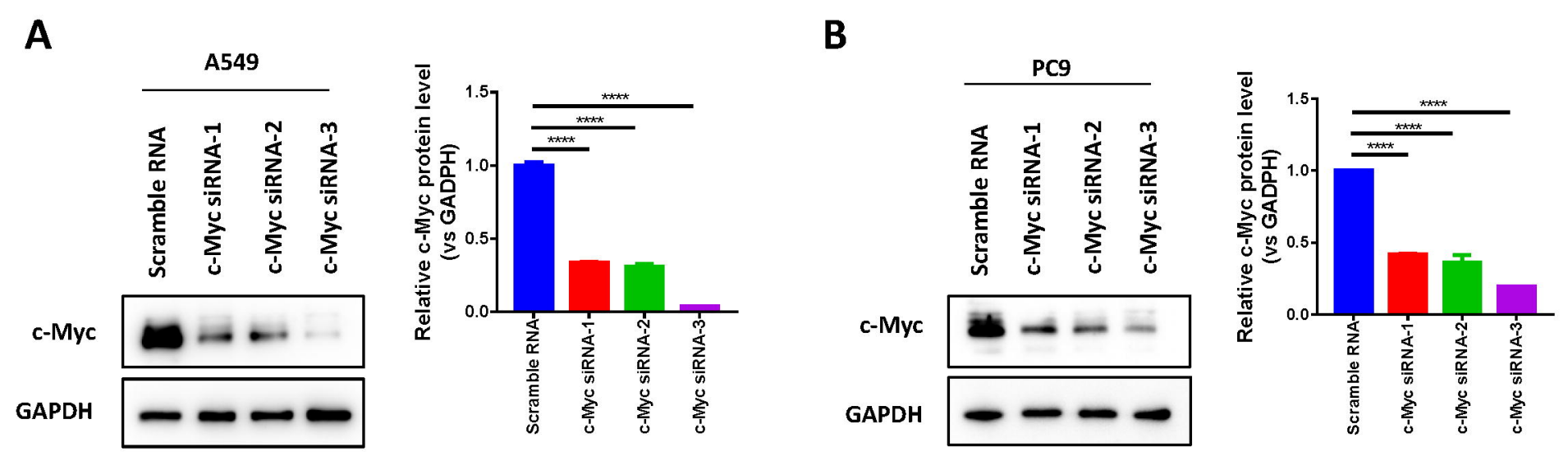
A

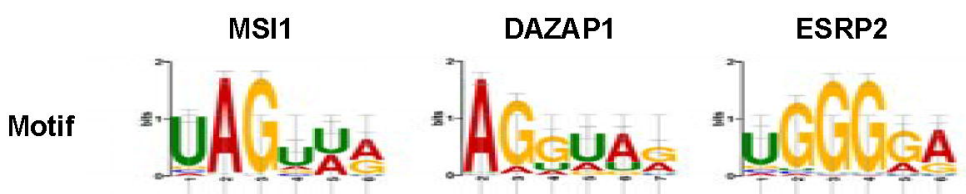

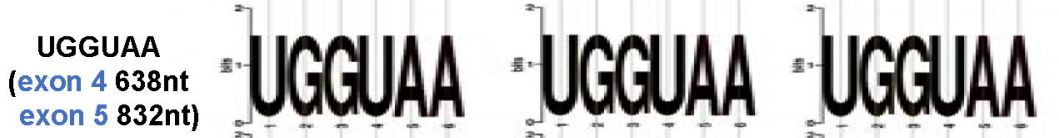

ascocas "AGGAGA "AGGAGA "AGGAGA

acosens "AGCGAG "AGGGAG "AGGGAG

B

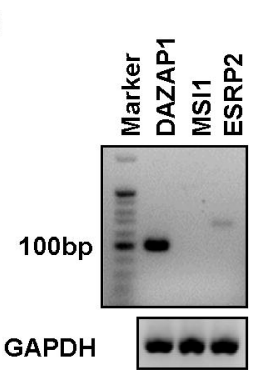

C
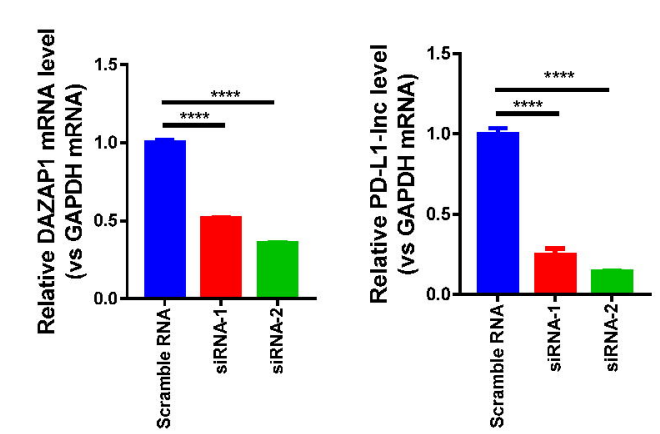